\title{
Kleinian Groups in Higher Dimensions
}

\author{
Michael Kapovich
}

To the memory of Sasha Reznikov

\begin{abstract}
This is a survey of higher-dimensional Kleinian groups, i.e., discrete isometry groups of the hyperbolic $n$-space $\mathbb{H}^{n}$ for $n \geq 4$. Our main emphasis is on the topological and geometric aspects of higher-dimensional Kleinian groups and their contrast with the discrete groups of isometry of $\mathbb{H}^{3}$.
\end{abstract}

Mathematics Subject Classification (2000). Primary 30F40; Secondary 20F67.

Keywords. Kleinian groups, hyperbolic manifolds.

\section{Introduction}

The goal of this survey is to give an overview (mainly from the topological perspective) of the theory of Kleinian groups in higher dimensions. The survey grew out of a series of lectures I gave in the University of Maryland in the Fall of 1991. An early (much shorter) version of this paper appeared as the preprint [110]. In this survey I collect well-known facts as well as less-known and new results. Hopefully, this will make the survey interesting to both non-experts and experts. We also refer the reader to Tukia's short survey [219] of higher-dimensional Kleinian groups.

There is a vast variety of Kleinian groups in higher dimensions: It appears that there is no hope for a comprehensive structure theory similar to the theory of discrete groups of isometries of $\mathbb{H}^{3}$. I do not know a good guiding principle for the taxonomy of higher-dimensional Kleinian groups. In this paper the higherdimensional Kleinian groups are organized according to the topological complexity of their limit sets. In this setting one of the key questions that I will address is the interaction between the geometry and topology of the limit set and the algebraic and topological properties of the Kleinian group.

During this work the I was partially supported by various NSF grants, especially DMS-8902619 at the University of Maryland and DMS-04-05180 at UC Davis. Most of this work was done when I was visiting the Max Plank Institute for Mathematics in Bonn. 
This paper is organized as follows. In Section 2 we consider the most basic concepts of the theory of Kleinian groups, e.g., domain of discontinuity, limit set, geometric finiteness, etc. In Section 3 we discuss various ways to construct Kleinian groups and list the tools of the theory of Kleinian groups in higher dimensions. In Section 4 we review the homological algebra used in the paper. In Section 5 we state topological rigidity results of Farrell and Jones and the coarse compact core theorem for higher-dimensional Kleinian groups. In Section 6 we discuss various notions of equivalence between Kleinian groups: From the weakest (isomorphism) to the strongest (conjugacy). In Section 7 we consider groups with zero-dimensional limit sets; such groups are relatively well understood. Convex-cocompact groups with 1-dimensional limit sets are discussed in Section 8. Although the topology of the limit sets of such groups is well understood, their group-theoretic structure is a mystery. We know very little about Kleinian groups with higher-dimensional limit sets, thus we restrict the discussion to Kleinian groups whose limit sets are topological spheres (Section 9). We then discuss Ahlfors finiteness theorem and its failure in higher dimensions (Section 10). We then consider the representation varieties of Kleinian groups (Section 11). Lastly we discuss algebraic and topological constraints on Kleinian groups in higher dimensions (Section 12).

Acknowledgments. I am grateful to C. McMullen, T. Delzant, A. Nabutovsky and J. Souto for several suggestions, and to L. Potyagailo for a number of comments, suggestions and corrections. I am also grateful to the referee for numerous corrections.

\section{Contents}

1. Introduction 485

2. Basic definitions 487

3. Ways and means of Kleinian groups 492

3.1. Ways: Sources of Kleinian groups 492

3.2. Means: Tools of the theory of Kleinian groups in higher dimensions 495

4. A bit of homological algebra 495

5. Topological rigidity and coarse compact core theorem 501

5.1. Results of Farrell and Jones 501

5.2. Limit sets and homological algebra $\quad 502$

5.3. Coarse compact core $\quad 502$

6. Notions of equivalence for Kleinian groups 504

7. Groups with zero-dimensional limit sets 508

8. Groups with one-dimensional limit sets 514

9. Groups whose limit sets are topological spheres $\quad 517$

9.1. Quasifuchsian groups of codimension 1 518

9.2. 1-quasifuchsian subgroups of $\operatorname{Mob}\left(\mathbb{S}^{3}\right) \quad 521$

10. Ahlfors finiteness theorem in higher dimensions:
Quest for the holy grail

10.1. The holy grail 524 
10.2. Groups with small limit sets $\quad 527$

11. Representation varieties of Kleinian groups 530

11.1. Local theory $\quad 531$

11.1.1. Small deformations of 1-quasifuchsian groups 532

11.1.2. Small deformations of $i$-quasifuchsian groups for $i \geq 2$

11.1.3. Failure of quasiconformal isotopy $\quad 538$

$\begin{array}{ll}\text { 11.2. Stability theorem } & 539\end{array}$

11.3. Space of discrete and faithful representations $\quad 541$

11.4. Why is it so difficult to construct higher-dimensional geometrically infinite Kleinian groups?

12. Algebraic and topological constraints on Kleinian groups 546

12.1. Algebraic constraints $\quad 546$

12.2. Topological constraints $\quad 550$

$\begin{array}{ll}\text { References } & 551\end{array}$

\section{Basic definitions}

Möbius transformations. For the lack of space, our discussion of the basics of Kleinian groups below is somewhat sketchy. For the detailed treatment we refer the reader to $[18,39,116,138,190]$. We let $\mathbb{B}^{n+1}$ denote the closed ball $\mathbb{H}^{n+1} \cup \mathbb{S}^{n}$; its boundary $\mathbb{S}^{n}$ is identified via the stereographic projection with $\overline{\mathbb{R}^{n}}=\mathbb{R}^{n} \cup\{\infty\}$. A horoball $B$ in $\mathbb{H}^{n+1}$ is a round ball in $\mathbb{H}^{n+1}$ which is tangent to the boundary sphere $\mathbb{S}^{n}$. The point of tangency is called the (hyperbolic) center of $B$.

Let $\operatorname{Mob}\left(\mathbb{S}^{n}\right)$ denote the group of all Möbius transformations of the $n$-sphere $\mathbb{S}^{n}$, i.e., compositions of inversions in $\mathbb{S}^{n}$. The group $\mathbf{M o b}\left(\mathbb{S}^{n}\right)$ admits an extension to the hyperbolic space $\mathbb{H}^{n+1}$, so that $\operatorname{Mob}\left(\mathbb{S}^{n}\right)=\operatorname{Isom}\left(\mathbb{H}^{n+1}\right)$, the isometry group of $\mathbb{H}^{n+1}$

For elements $\gamma \in \operatorname{Mob}\left(\mathbb{S}^{n}\right)$ define the displacement function

$$
d_{\gamma}(x):=d(x, \gamma(x)), \quad x \in \mathbb{H}^{n+1} .
$$

The elements $\gamma$ of $\mathbf{M o b}\left(\mathbb{S}^{n}\right)$ are classified as:

1. Hyperbolic: The function $d_{\gamma}$ is bounded away from zero. Its minimum is attained on a geodesic $A_{\gamma} \subset \mathbb{H}^{n+1}$ invariant under $\gamma$. The ideal end-points of $A_{\gamma}$ are the fixed points of $\gamma$ in $\mathbb{S}^{n}$.

2. Parabolic: The function $d_{\gamma}$ is positive but has zero infimum on $\mathbb{H}^{n+1}$; such elements have precisely one fixed point in $\mathbb{S}^{n}$.

3. Elliptic: $\gamma$ fixes a point in $\mathbb{H}^{n+1}$.

The group $\operatorname{Mob}\left(\mathbb{S}^{n}\right)$ is isomorphic to an index 2 subgroup in the Lorentz group $O(n+1,1)$, see, e.g., [190]. In particular, $\mathbf{M o b}\left(\mathbb{S}^{n}\right)$ is a matrix group. Selberg's lemma [203] implies that every finitely generated group of matrices contains a finite index subgroup which is torsion-free. A group $\Gamma$ is said to virtually satisfy a property $X$ if it contains a finite index subgroup $\Gamma^{\prime} \subset \Gamma$, such that $\Gamma^{\prime}$ satisfies $X$. 
Therefore, every finitely generated group of matrices is virtually torsion-free. Moreover, every finitely-generated matrix group is residually finite, i.e., the intersection of all its finite-index subgroups is trivial, see [151, 203]. This, of course, applies to the finitely generated subgroups of $\mathbf{M o b}\left(\mathbb{S}^{n}\right)$ as well.

Definition 2.1. A discrete subgroup $\Gamma \subset \operatorname{Mob}\left(\mathbb{S}^{n}\right)$ is called a Kleinian group.

Dynamical notions. The discontinuity set $\Omega(\Gamma)$ of a group $\Gamma \subset \operatorname{Mob}\left(\mathbb{S}^{n}\right)$, is the largest open subset in $\mathbb{S}^{n}$ where $\Gamma$ acts properly discontinuously. Its complement $\mathbb{S}^{n} \backslash \Omega(\Gamma)$ is the limit set $\Lambda(\Gamma)$ of the group $\Gamma$. Equivalently, the limit set of a Kleinian group can be described as the accumulation set in the sphere $\mathbb{S}^{n}$ of an orbit $\Gamma \cdot o$. Here $o$ is an arbitrary point in $\mathbb{H}^{n+1}$. A Kleinian group is called elementary if its limit set is finite, i.e., is either empty, or consists of one or of two points.

We will use the notation $M^{n}(\Gamma)$ for the $n$-dimensional quotient $\Omega(\Gamma) / \Gamma$ and $\bar{M}^{n+1}(\Gamma)$ for the $n+1$-dimensional quotient $\left(\mathbb{H}^{n+1} \cup \Omega(\Gamma)\right) / \Gamma$.

For a closed subset $\Lambda \subset \mathbb{S}^{n}$, let $\operatorname{Hull}(\Lambda)$ denote its convex hull in $\mathbb{H}^{n+1}$, i.e., the smallest closed convex subset $H$ of $\mathbb{H}^{n+1}$ such that

$$
c l_{\mathbb{B}^{n+1}}(H) \cap \mathbb{S}^{n}=\Lambda .
$$

Clearly, if $\Lambda$ is a point, then $\operatorname{Hull}(\Lambda)$ does not exist. Otherwise, $\operatorname{Hull}(\Lambda)$ exists and is unique. We declare $\operatorname{Hull}(\Lambda)$ to be empty in the case when $\Lambda$ is a single point.

One way to visualize the convex hull $\operatorname{Hull}(\Lambda)$ is to consider the projective model of the hyperbolic space, where the geodesic lines are straight line segments contained in the interior of $\mathbb{B}^{n+1}$. Therefore, the Euclidean notion of convexity coincides with the hyperbolic notion. This implies that the convex hull in this model can be described as follows: $\operatorname{Hull}(\Lambda)$ is the intersection of the Euclidean convex hull of $\Lambda$ with the interior of $\mathbb{B}^{n+1}$.

Suppose that $\Lambda=\Lambda(\Gamma)$ is the limit set of a Kleinian group $\Gamma \subset \operatorname{Mob}\left(\mathbb{S}^{\mathrm{n}}\right)$. The quotient $\operatorname{Hull}(\Lambda) / \Gamma$ is called the convex core of the orbifold $N=\mathbb{H}^{n+1} / \Gamma$. It is characterized by the property that it is the smallest closed convex subset in $N$, whose inclusion to $N$ is a homotopy-equivalence. For $\epsilon>0$ consider the open $\epsilon$-neighborhood $\operatorname{Hull}_{\epsilon}(\Lambda)$ of $H u l l(\Lambda)$ in $\mathbb{H}^{n+1}$. Since $H u l l_{\epsilon}(\Lambda)$ is $\Gamma$-invariant, we can form the quotient $\operatorname{Hull}_{\epsilon}(\Lambda) / \Gamma$. Then $H_{u l l}(\Lambda) / \Gamma$ is the $\epsilon$-neighborhood of the convex core.

Geometric finiteness. We now arrive to one of the key notions in the theory of Kleinian groups:

Definition 2.2. A Kleinian group $\Gamma \subset \operatorname{Mob}\left(\mathbb{S}^{n}\right)$ is called geometrically finite if:

(1) $\Gamma$ is finitely generated, and

(2) $\operatorname{vol}\left(\operatorname{Hull}_{\epsilon}(\Lambda(\Gamma)) / \Gamma\right)<\infty$.

In a number of important special cases, e.g., when $\Gamma$ is torsion-free, or $n=2$, or when $\Lambda(\Gamma)=\mathbb{S}^{n}$, the assumption (1) follows from (2), see [39]. However, E. Hamilton [93] constructed an example of a Kleinian group $\Gamma \subset \mathbf{M o b}\left(\mathbb{S}^{3}\right)$ for which 
(2) holds but (1) fails. This group contains finite order elements of arbitrarily high order; by Selberg's lemma such groups cannot be finitely-generated.

A Kleinian group $\Gamma \subset \operatorname{Mob}\left(\mathbb{S}^{n}\right)$ is called a lattice if $\mathbb{H}^{n+1} / \Gamma$ has finite volume. Equivalently, $\Lambda(\Gamma)=\mathbb{S}^{n}$ and $\Gamma$ is geometrically finite. A lattice is cocompact (or uniform) if $\mathbb{H}^{n+1} / \Gamma$ is compact.

One can characterize geometrically finite groups in terms of their limit sets. Before stating this theorem we need two more definitions.

Definition 2.3. A limit point $\xi \in \Lambda(\Gamma)$ is called a conical limit point if there exists a geodesic $\alpha \subset \mathbb{H}^{n+1}$ asymptotic to $\xi$, a point $o \in \mathbb{H}^{n+1}$, a number $r<\infty$, and a sequence $\gamma_{i} \in \Gamma$ so that

1. $\lim _{i} \gamma_{i}(o)=\xi$.

2. $d\left(\gamma_{i}(o), \alpha\right) \leq r$.

The reason for this name comes from the shape of the $r$-neighborhood of the vertical geodesic $\alpha$ in the upper half-space model of $\mathbb{H}^{n+1}$ : It is a Euclidean cone with the axis $\alpha$. Equivalently, one can describe the conical limit points of nonelementary groups as follows (see [14, 39]):

$\xi \in \Lambda(\Gamma)$ is a conical limit point if and only if for every $\eta \in \Lambda(\Gamma) \backslash\{\xi\}$ there exists a point $\psi$ and a sequence $\gamma_{i} \in \Gamma$ such that:

1. $\lim _{i} \gamma_{i}(\zeta)=\xi$ for every $\zeta \in \Lambda(\Gamma) \backslash\{\psi\}$.

2. $\lim _{i} \gamma_{i}^{-1}(\xi) \neq \lim _{i} \gamma_{i}^{-1}(\eta)$.

The set of conical limit points of a Kleinian group $\Gamma$ is denoted $\Lambda_{c}(\Gamma)$.

Definition 2.4. A point $\xi \in \Lambda(\Gamma)$ is called a bounded parabolic point if it is the fixed point of a parabolic subgroup $\Pi \subset \Gamma$ and $(\Lambda(\Gamma)-\{\xi\}) / \Pi$ is compact.

Below is a dynamical characterizations of geometrically finite groups:

Theorem 2.5. (A. Beardon and B. Maskit [14], B. Bowditch [39]) A Kleinian group $\Gamma$ is geometrically finite if and only if each limit point $\xi \in \Lambda(\Gamma)$ is either a conical limit point or a bounded parabolic point.

C. Bishop [32] proved that one can drop the word bounded in the above theorem. We refer the reader to Bowditch's paper [39] for the proof of other criteria of geometric finiteness collected in Theorems 2.6, 2.7, 2.8 below. (The case $n=2$ is treated in [152] and [213].)

Theorem 2.6. $\quad$ 1. If a Kleinian subgroup $\Gamma \subset \operatorname{Mob}\left(\mathbb{S}^{n}\right)$ admits a convex fundamental polyhedron with finitely many faces then it is geometrically finite.

2. Let $\Gamma \subset \operatorname{Mob}\left(\mathbb{S}^{n}\right)$ be a geometrically finite Kleinian group so that either (a) $n \leq 2$, or (b) $\Gamma$ contains no parabolic elements, or (c) $\Gamma$ is a lattice.

Then $\Gamma$ admits a convex fundamental polyhedron with finitely many faces.

On the other hand, there are geometrically finite subgroups of $\operatorname{Mob}\left(\mathbb{S}^{3}\right)$ which do not admit a convex fundamental polyhedron with finitely many faces, see $[10]$. 
Theorem 2.7. Let $\Gamma \subset \operatorname{Mob}\left(\mathbb{S}^{n}\right)$ be a Kleinian subgroup containing no parabolic elements. Then the following are equivalent:

(a) $\Gamma$ is geometrically finite.

(b) $\operatorname{Hull}(\Lambda(\Gamma)) / \Gamma$ is compact.

(c) $\bar{M}^{n+1}(\Gamma)$ is compact.

If $\Gamma$ is geometrically finite and contains no parabolic elements, it is called convex-cocompact. We will frequently use the fact that every convex-cocompact Kleinian group is Gromov-hyperbolic, see, e.g., [44].

The criterion given in Theorem 2.7 generalizes to the case of groups with parabolic elements, although the statement becomes more complicated:

Theorem 2.8. The following are equivalent:

(a) $\Gamma$ is geometrically finite.

(b) There exists a pairwise disjoint $\Gamma$-invariant collection of open horoballs $B_{i} \subset$ $\mathbb{H}^{n+1}, i \in I$, which are centered at fixed points of parabolic subgroups of $\Gamma$, such that the quotient

$$
\left(\operatorname{Hull}(\Lambda(\Gamma)) \backslash \bigcup_{i \in I} B_{i}\right) / \Gamma
$$

is compact.

(c) Let $\Pi_{i}, i \in I$ be the collection of maximal (virtually) parabolic subgroups of $\Gamma$. For each $i$ there exists a $\Pi_{i}$-invariant convex subset $C_{i} \subset \mathbb{B}^{n+1}$, so that the quotient

$$
\left(\mathbb{H}^{n+1} \cup \Omega(\Gamma) \backslash \bigcup_{i \in I} C_{i}\right) / \Gamma
$$

is compact. If $\Omega(\Gamma)=\emptyset$, then one can take $C_{i}=B_{i}$, a horoball in $\mathbb{H}^{n+1}$.

If $n=1$, then every finitely generated Kleinian group is geometrically finite. The proof is rather elementary, see, e.g., [53]. For $n \geq 2$ this implication is no longer true. The first (implicit) examples were given by L. Bers, they are singlydegenerate groups:

Definition 2.9. A finitely generated nonelementary Kleinian subgroup of $\mathbf{M o b}\left(\mathbb{S}^{2}\right)$ is singly degenerate if its domain of discontinuity is simply-connected, i.e., homeomorphic to the 2-disk.

L. Bers [19] proved that singly degenerate Kleinian groups exist and are never geometrically finite. The first explicit examples of finitely generated geometrically infinite Kleinian subgroups $\Gamma$ of $\operatorname{Mob}\left(\mathbb{S}^{2}\right)$ were given by T. Jørgensen [104]. In Jørgensen's examples, $\Gamma$ appears as a normal subgroup of a lattice $\hat{\Gamma} \subset \operatorname{Mob}\left(\mathbb{S}^{2}\right)$ with $\hat{\Gamma} / \Gamma \cong \mathbb{Z}$. Remarkably, all known examples of finitely-generated geometrically infinite Kleinian subgroups of $\mathbf{M o b}\left(\mathbb{S}^{n}\right)$ can be traced to the 2-dimensional examples. More precisely, every known finitely-generated geometrically infinite Kleinian 
subgroup $\Gamma \subset \operatorname{Mob}\left(\mathbb{S}^{n}\right)$ admits a decomposition as the graph of groups

$$
\left(\mathcal{G}, \Gamma_{v}, \Gamma_{e}\right)
$$

where at least one of the vertex groups $\Gamma_{v}$ is either a geometrically infinite subgroup contained in $\operatorname{Mob}\left(\mathbb{S}^{2}\right)$, or is a quasiconformal deformations of such.

Problem 2.10. Construct examples of finitely-generated geometrically infinite subgroups of $\operatorname{Mob}\left(\mathbb{S}^{n}\right), n \geq 3$, which do not have the 2-dimensional origin as above.

Assumption 2.11. From now on we will assume that all Kleinian groups are finitely generated and torsion-free, unless stated otherwise.

Note that the second part of this assumption is not very restrictive because of Selberg's lemma.

Cusps and tubes. The $\Gamma$-conjugacy classes $[\Pi]$ of maximal parabolic subgroups $\Pi$ of a Kleinian group $\Gamma$ are called cusps of $\Gamma$. More geometrically, cusps of $\Gamma$ can be described using the thick-thin decomposition of the quotient manifold $M=$ $\mathbb{H}^{n+1} / \Gamma$. Given a positive number $\epsilon>0$, let $M_{(0, \epsilon]}$ denote the collection of points $x$ in $M$ such that there exists a homotopically nontrivial loop $\alpha$ based at $x$, so that the length of $\alpha$ is at most $\epsilon$. Then $M_{(\epsilon, \infty)}$ is the complement of $M_{(0, \epsilon]}$ in $M$. According to Kazhdan-Margulis lemma [130], there exists a number $\mu=\mu_{n+1}>0$ such that for every Kleinian group $\Gamma$ and every $0<\epsilon \leq \mu$, every component of $M_{(0, \epsilon]}$ has a virtually abelian fundamental group. The submanifold $M_{(0, \epsilon]}$ is called the thin part of $M$ and its complement the thick part of $M$. The compact components of $M_{(0, \epsilon]}$ are called tubes and the noncompact components are called cusps.

Then the cusps of $\Gamma$ are in bijective correspondence with the cusps in $M_{(0, \epsilon]}$ :

For every cusp $[\Pi]$ in $\Gamma$, there exists a noncompact component $C \subset M_{(0, \epsilon]}$, so that $\Pi=\pi_{1}(C)$. Conversely, for each cusp $C \subset M$, there exists a maximal parabolic subgroup $\Pi \subset \Gamma$ such that $\Pi=\pi_{1}(C)$.

Taking the $\Gamma$-conjugacy class of $\Pi$ reflects the ambiguity in the choice of the basepoint needed to identify $\pi_{1}(M)$ and $\Gamma$.

If $n \leq 2$ and the manifold $M$ is oriented, then the components $C_{i}$ of $M_{(0, \epsilon]}$ are convex: The cusps in $M$ are quotients of horoballs in $\mathbb{H}^{n+1}$, while the compact components $T_{i}$ of $M_{(0, \epsilon]}$ are metric $R_{i}$-neighborhoods of closed geodesics $\gamma_{i} \subset M$. In higher dimensions $(n \geq 3)$ convexity (in general) fails. However every tube $T_{i}$ in $M_{(0, \epsilon]}$ is a finite union of convex sets containing a certain closed geodesic $\gamma_{i} \subset T_{i}$. In particular, every tube $T_{i}$ is homeomorphic to a disk bundle over $\mathbb{S}^{1}$. A similar, although more complicated description, holds for the cusps, where one has to consider (in general) a union of infinitely many convex subsets. See, e.g., [121].

Möbius structures. In this paper we shall also discuss the subject closely related to the theory of Kleinian groups, namely Möbius structures. When $M$ is a smooth manifold of dimension $\geq 3$, a Möbius (or flat conformal) structure $K$ on a $M$ is 
the conformal class of a conformally-Euclidean Riemannian metric on $M$. Topologically, $K$ is a maximal Möbius atlas on $M$, i.e., an atlas with Möbius transition maps. Thus, for each Kleinian group $\Gamma$ and $\Gamma$-invariant subset $\Omega \subset \Omega(\Gamma)$, the standard Möbius structure on $\Omega \subset \mathbb{S}^{n}$ projects to a Möbius structure $K_{\Gamma}$ on the manifold $\Omega / \Gamma$. The Möbius structures of this type are called uniformizable.

Complex-hyperbolic Kleinian groups. Instead of considering the isometry group of the hyperbolic space, one can consider other negatively curved symmetric spaces, for instance, the complex-hyperbolic $n$-space $\mathbb{C H}^{n}$ and its group of automorphisms $P U(n, 1)$. From the analytical viewpoint, $\mathbb{C H}^{n}$ is the unit ball in $\mathbb{C}^{n}$ and $P U(n, 1)$ is the group of biholomorphic automorphisms of this ball. The Bergman metric on $\mathbb{C H}^{n}$ is a Kähler metric of negative sectional curvature. The discrete subgroups of $P U(n, 1)$ are complex-hyperbolic Kleinian groups. They share many properties with Kleinian groups. In fact, nearly all positive results stated in this survey for Kleinian subgroups of $\operatorname{Mob}\left(\mathbb{S}^{n}\right)(n \geq 3)$ are also valid for the complex-hyperbolic Kleinian groups! (One has to replace virtually abelian with virtually nilpotent in the discussion of cusps.) There exists an isometric embedding $\mathbb{H}^{n} \rightarrow \mathbb{C} \mathbb{H}^{n}$ which induces an embedding of the isometry groups and therefore complex-hyperbolic Kleinian groups $(n \geq 4)$ also inherit the pathologies of the higher-dimensional Kleinian groups. We refer the reader to $[80,81,199]$ for detailed discussion.

\section{Ways and means of Kleinian groups}

\subsection{Ways: Sources of Kleinian groups}

The following is a list of ways to construct Kleinian groups.

(a) Poincaré fundamental polyhedron theorem (see, e.g., [190] for a very detailed discussion, as well as [157]). This source is, in principle, the most general. The Poincaré fundamental polyhedron theorem asserts that given a polyhedron $\Phi$ in $\mathbb{H}^{n+1}$ and a collection of elements $\gamma_{1}, \gamma_{2}, \ldots, \gamma_{k}, \ldots$ of $\operatorname{Mob}\left(\mathbb{S}^{n}\right)$, pairing the faces of $\Phi$, under certain conditions on this data, the group $\Gamma$ generated by $\gamma_{1}, \gamma_{2}, \ldots, \gamma_{k}, \ldots$ is Kleinian and $\Phi$ is a fundamental domain for the action of the group $\Gamma$ on $\mathbb{H}^{n+1}$.

Every Kleinian group has a convex fundamental polyhedron (for example, the Dirichlet fundamental domain). However, in practice, the Poincaré fundamental polyhedron theorem is not always easy to use, especially if $\Phi$ has many faces and $n$ is large. This theorem was used, for instance, to construct non-arithmetic lattices in $\operatorname{Mob}\left(\mathbb{S}^{n}\right)$ (see $\left.[149,150,221]\right)$, as well as other interesting Kleinian groups, see, e.g., $[60,98,111,139,191]$.

(b) Klein-Maskit Combination Theorems (see, e.g., [138] and [157]). Suppose that we are given two Kleinian groups $\Gamma_{1}, \Gamma_{2} \subset \operatorname{Mob}\left(\mathbb{S}^{n}\right)$ which share a common subgroup $\Gamma_{3}$, or a single Kleinian group $\Gamma_{1}$ and a Möbius transformation $\tau \in$ $\operatorname{Mob}\left(\mathbb{S}^{\mathrm{n}}\right)$ which conjugates subgroups $\Gamma_{3}, \Gamma_{3}^{\prime} \subset \Gamma_{1}$. The Combination Theorems 
provide conditions which guarantee that the group $\Gamma \subset \operatorname{Mob}\left(\mathbb{S}^{n}\right)$ generated by $\Gamma_{1}$ and $\Gamma_{2}$ (or by $\Gamma_{1}$ and $\tau$ ) is again Kleinian and is isomorphic to the amalgam

$$
\Gamma \cong \Gamma_{1} *_{\Gamma_{3}} \Gamma_{2},
$$

or to the HNN extension

$$
\Gamma \cong \Gamma_{1} *_{\Gamma_{3}}=H N N\left(\Gamma_{1}, \tau\right) .
$$

The proofs of the Combination Theorems generalize the classical "ping-pong" argument due to Schottky and Klein. The Combination Theorems also show that the quotient manifold $M^{n}(\Gamma)$ of the group $\Gamma$ is obtained from $M^{n}\left(\Gamma_{1}\right), M^{n}\left(\Gamma_{2}\right)$ (or $\left.M\left(\Gamma_{1}\right)\right)$ via some "cut-and-paste" operation. Moreover, Combination Theorems generalize to graph of groups. There should be a generalization of Combination Theorems to complexes of groups (see, e.g., [44] for the definition); however, to the best of my knowledge, nobody worked out the general result, see [118] for a special case.

(c) Arithmetic groups and their subgroups (see, e.g., [148] and [222]). A subgroup $\Gamma \subset O(n, 1)$ is called arithmetic if there exists an embedding

$$
\iota: O(n, 1) \hookrightarrow G L(N, \mathbb{R}),
$$

such that the image $\iota(\Gamma)$ is commensurable with the intersection

$$
\iota(O(n, 1)) \cap G L(N, \mathbb{Z}) .
$$

Recall that two subgroups $\Gamma_{1}, \Gamma_{2} \subset G$ are called commensurable if $\Gamma_{1} \cap \Gamma_{2}$ has finite index in both $\Gamma_{1}$ and $\Gamma_{2}$.

Below is a specific construction of arithmetic groups. Let $f$ be a quadratic form of signature $(n, 1)$ in $n+1$ variables with coefficients in a totally real algebraic number field $K \subset \mathbb{R}$ satisfying the following condition:

(*) For every nontrivial (i.e., different from the identity) embedding $\sigma: K \rightarrow \mathbb{R}$, the quadratic form $f^{\sigma}$ is positive definite.

Without loss of generality one may assume that this quadratic form is diagonal. For instance, take

$$
f(x)=-\sqrt{2} x_{0}^{2}+x_{1}^{2}+\cdots+x_{n}^{2} .
$$

We now define discrete subgroups of $\operatorname{Isom}\left(\mathbb{H}^{n}\right)$ using the form $f$. Let $A$ denote the ring of integers of $K$. We define the group $\Gamma:=O(f, A)$ consisting of matrices with entries in $A$ preserving the form $f$. Then $\Gamma$ is a discrete subgroup of $O(f, \mathbb{R})$. Moreover, it is a lattice: its index 2 subgroup

$$
\Gamma^{\prime}=O^{\prime}(f, A):=O(f, A) \cap O^{\prime}(f, \mathbb{R})
$$

acts on $\mathbb{H}^{n}$ so that $\mathbb{H}^{n} / \Gamma^{\prime}$ has finite volume. Such groups $\Gamma$ (and subgroups of $\operatorname{Isom}\left(\mathbb{H}^{n}\right)$ commensurable to them) are called arithmetic subgroups of the simplest type in $O(n, 1)$, see [222]. 
Remark 3.1. If $\Gamma \subset O(n, 1)$ is an arithmetic lattice so that either $\Gamma$ is noncocompact or $n$ is even, then it follows from the classification of rational structures on $O(n, 1)$ that $\Gamma$ is commensurable to an arithmetic lattice of the simplest type. For odd $n$ there is another family of arithmetic lattices given as the groups of units of appropriate skew-Hermitian forms over quaternionic algebras. Yet other families of arithmetic lattices exist for $n=3$ and $n=7$. See, e.g., [222].

We refer the reader to [148] for the detailed treatment of geometry and topology of arithmetic subgroups of $\operatorname{Mob}\left(\mathbb{S}^{2}\right)$.

(d) Small deformations of a given Kleinian group. We discuss this construction in detail in Section 11.1. The idea is to take a Kleinian group $\Gamma \subset \operatorname{Mob}\left(\mathbb{S}^{n}\right)$ and to "perturb it a little bit", by modifying the generators slightly (within $\operatorname{Mob}\left(\mathbb{S}^{n}\right)$ ) and preserving the relators. The result is a new group $\Gamma^{\prime}$ which may or may not be Kleinian and even if it is, $\Gamma^{\prime}$ is not necessarily isomorphic to $\Gamma$. However if $\Gamma$ is convex-cocompact, $\Gamma^{\prime}$ is again a convex-cocompact group isomorphic to $\Gamma$, see Theorem 11.12 .

(e) Limits of sequences of Kleinian groups, see Section 11.3. Take a sequence $\Gamma_{i}$ of Kleinian subgroups of $\operatorname{Mob}\left(\mathbb{S}^{n}\right)$ and assume that it has a limit $\Gamma$ : It turns out that there are two ways to make sense of this procedure (algebraic and geometric limit). In any case, $\Gamma$ is again a Kleinian group. Even if the (algebraic) limit does not exist as a subgroup of $\operatorname{Mob}\left(\mathbb{S}^{n}\right)$, there is a way to make sense of the limiting group as a group of isometries of a metric tree. This logic turns out to be useful for proving compactness theorems for sequences of Kleinian groups.

(f) Differential-geometric constructions of hyperbolic metrics. The only (but spectacular) example where it has been used is Perelman's work on Ricci flow and proof of Thurston's geometrization conjecture. See [134, 173, 183, 184]. However applicability of this tool at the moment appears to be limited to 3-manifolds.

A beautiful example of application of (b) and (c) is the construction of M. Gromov and I. Piatetski-Shapiro [88] of non-arithmetic lattices in $\operatorname{Mob}\left(\mathbb{S}^{\mathrm{n}}\right)$. Starting with two arithmetic groups $\Gamma_{j}(j=1,2)$ they first "cut these groups in half", take "one half" $\Delta_{j} \subset \Gamma_{j}$ of each, and then combine $\Delta_{1}$ and $\Delta_{2}$ via Maskit Combination. The construction of Kleinian groups in [89] (see also Section 9.1) is an application of (b), (c) and (d). Thurston's hyperbolic Dehn surgery theorem is an example of (e). One of the most sophisticated constructions of Kleinian groups is given by Thurston's hyperbolization theorem (see, e.g., [116], [180], [181]); still, it is essentially a combination (a very complicated one!) of (b), (d) and (e).

Remark 3.2. There is potentially the sixth source of Kleinian groups in higher dimensions: monodromy of linear ordinary differential equations. However, to the best of my knowledge, the only example of its application relevant to Kleinian groups, is the construction of lattices in $P U(n, 1)$ (i.e., the isometry group of the complex-hyperbolic $n$-space) by Deligne and Mostow, see [62]. 


\subsection{Means: Tools of the theory of Kleinian groups in higher dimensions}

Several key tools of the "classical" theory of Kleinian subgroups of $\operatorname{Mob}\left(\mathbb{S}^{2}\right)$ (mainly, the Beltrami equation and pleated hypersurfaces) are missing in higher dimensions. Below is the list of main tools that are currently available.

(a) Dynamics, more specifically, the convergence property. Namely, every sequence of Möbius transformations $\gamma_{i} \in \operatorname{Mob}\left(\mathbb{S}^{n}\right)$ either contains a convergent subsequence or contains a subsequence which converges to a constant map away from a point in $\mathbb{S}^{n}$. See, e.g., [116].

(b) Kazhdan-Margulis lemma and its corollaries.

It turns out that the lion share of the general results about higher-dimensional Kleinian groups is a combination of (a) and (b), together with some hyperbolic geometry.

(c) Group actions on trees and Rips theory. This is a very potent tool for proving compactness results for families of representations of Kleinian groups, see for instance Theorem 11.16.

(d) Barycentric maps. These maps were originally introduced by A. Douady and C. Earle [65] as a tool of the Teichmüller theory of Riemann surfaces. In the hands of G. Besson, G. Courtois and S. Gallot these maps became a powerful analytic tool of the theory of Kleinian groups in higher dimensions, see, e.g., [21, 22], as well as Theorems 10.21 and 11.24 in this survey. In contrast, equivariant harmonic maps which proved so useful in the study of, say, Kähler groups, seem at the moment to be only of a very limited use in the theory of Kleinian groups in higher dimensions.

(e) Ergodic theory of the actions of $\Gamma$ on its limit set and Patterson-Sullivan measures. See for instance [177, 208] and the survey of P. Tukia [219].

(f) Conformal geometric analysis. This is a branch of (conformal) differential geometry concerned with the analysis of the conformally-flat Riemannian metrics on $M^{n}(\Gamma)=\Omega(\Gamma) / \Gamma$. This tool tends to work rather well in the case when $M^{n}(\Gamma)$ is compact. The most interesting examples of this technique are due to R. Schoen and S-T. Yau [198], S. Nayatani [176], A. Chang, J. Qing, J. and P. Yang, [54], and H. Izeki [100, 101, 102].

(g) Infinite-dimensional representation theory of the group $\operatorname{Mob}\left(\mathbb{S}^{n}\right)$. The only (but rather striking) example of its application is Y. Shalom's work [204].

(h) Topological rigidity theorems of Farrell and Jones: See Section 5.

\section{A bit of homological algebra}

Why does one need homological algebra in order to study higher-dimensional Kleinian groups? 
Essentially the only time one encounters group cohomology with twisted coefficients in the study of Kleinian subgroups of $\operatorname{Mob}\left(\mathbb{S}^{2}\right)$, is in the proof of Ahlfors' finiteness theorem, see [136]. Another (minor) encounter appears in the proof of the smoothness theorem for deformation spaces of Kleinian groups, Section 8.8 in [116]. Otherwise, homological algebra is hardly ever needed. The main reason for this, I believe, is 3-fold:

1. Solvability of the 2-dimensional Beltrami equation, which implies smoothness of the deformation spaces of Kleinian groups in the most interesting situations.

2. Scott compact core theorem [201, 202] ensures that every finitely-generated Kleinian group $\Gamma \subset \operatorname{Mob}\left(\mathbb{S}^{2}\right)$ satisfies a very strong finiteness property: Not only it is finitely-presented, it is also (canonically) isomorphic to the fundamental group of a compact aspherical 3-manifold with boundary (Scott compact core).

3. The separation between Kleinian groups of the cohomological dimension 1 , 2 and 3 comes rather easily: Free groups, "generic" Kleinian groups, and lattices. Moreover, every Kleinian group $\Gamma \subset \mathbf{M o b}\left(\mathbb{S}^{2}\right)$ which is not a lattice, splits as

$$
\Gamma \cong \Gamma_{0} * \Gamma_{1} * \cdots * \Gamma_{k}
$$

where $\Gamma_{0}$ is free and each $\Gamma_{i}, i \geq 1$, is freely indecomposable, 2-dimensional group. In the language of homological algebra, the group $\Gamma_{0}$ has cohomological dimension 1 , while the groups $\Gamma_{i}, i \geq 1$, are two-dimensional duality groups.

All this changes rather dramatically in higher dimensions:

1. Solvability of the Beltrami equation fails, which, in particular, leads to non-smoothness of the deformation spaces of Kleinian groups, Theorem 11.4. In order to study the local structure of character varieties one then needs the first and the second group cohomology with (finite-dimensional) twisted coefficients.

2. Scott compact core theorem fails for Kleinian subgroups of $\operatorname{Mob}\left(\mathbb{S}^{3}\right)$, for instance, they do not have to be finitely-presented, see Section 10. Therefore, it appears that one has to reconsider the assumption that Kleinian groups are finitely-generated. It is quite likely, that in higher dimensions, in order to get good structural results, one has to restrict to Kleinian groups of finite type, i.e., type $F P$, defined below. This definition requires homological algebra.

3 . One has to learn how to separate $k$-dimensional from $m$-dimensional in the algebraic structure of Kleinian groups. For the subgroups of $\operatorname{Mob}\left(\mathbb{S}^{2}\right)$ this separation comes in the form of the free product decomposition (4.1). It appears at the moment that "truly" $m$-dimensional groups are the $m$-dimensional duality groups. For instance, for Kleinian subgroups $\Gamma$ of $\operatorname{Mob}\left(\mathbb{S}^{n}\right)$ which are $n$-dimensional duality groups, one can prove a coarse form of the Scott compact core theorem, [124]. In particular, every such group admits the structure of an $n+1$-dimensional Poincaré duality pair $(\Gamma, \Delta)$. The latter is a homological analogue of the fundamental group of a compact aspherical $n+1$-manifold with boundary (where the boundary corresponds to the collection of subgroups $\Delta$ in $\Gamma$ ). See Section 5.3 for more details. 
4. The (co)homological dimension appears to be an integral part of the discussion of the critical exponent of higher-dimensional Kleinian groups, see Section 10.2 and Izeki's papers $[100,101]$.

(Co)homology of groups. Some of the above discussion was rather speculative; we now return to the firm ground of homological algebra. We refer the reader to $[29,47]$ for the comprehensive treatment of (co)homologies of groups.

Throughout this section we let $R$ be a commutative ring with a unit. The examples that the reader should have in mind are $R=\mathbb{Z}, \mathbb{Z} / p \mathbb{Z}$ and $R=\mathbb{R}$. The group ring $R \Gamma$ of a group $\Gamma$ consists of finite linear combinations of the form

$$
\sum_{\gamma \in \Gamma} r_{\gamma} \gamma
$$

with $r_{\gamma} \in R$ equal to zero for all but finitely many $\gamma \in \Gamma$. Let $V$ be a (left) $R \Gamma$ module. Basic examples include $V=R$ (with the trivial $R \Gamma$-module structure) and $V=R \Gamma$. If $R$ is a field, then $V$ is nothing but a vector space over $R$ equipped with a linear action of the group $\Gamma$. The very useful (for the theory of Kleinian groups) example is the following:

Let $G=\operatorname{Mob}\left(\mathbb{S}^{n}\right), \mathfrak{g}$ be the Lie algebra of $G$. Then $G$ acts on $\mathfrak{g}$ via the adjoint representation $A d=A d_{G}$. Therefore $\mathfrak{g}$ becomes an $\mathbb{R} G$-module. For every abstract group $\Gamma$ and a representation $\rho: \Gamma \rightarrow G$ we obtain the $\mathbb{R} \Gamma$-module

$$
V=\mathfrak{g}_{A d(\rho)},
$$

where the action of $\Gamma$ is given by the composition $A d \circ \rho$. We will abbreviate this module to $A d(\rho)$. From the theory of Kleinian groups viewpoint, the most important example of this module is when $\Gamma$ is a Kleinian subgroup of $G$ and $\rho$ is the identity embedding.

A projective $R \Gamma$-module, is a module $P$, such that every exact sequence of $R \Gamma$-modules

$$
Q \rightarrow P \rightarrow 0
$$

splits. For instance, every free $R \Gamma$-module is projective.

Assume now that $V$ be an $R \Gamma$-module. A resolution of $V$ is an exact sequence of $R \Gamma$-modules:

$$
\cdots \rightarrow P_{n} \rightarrow \cdots \rightarrow P_{0} \rightarrow V \rightarrow 0 .
$$

Every $R \Gamma$-module has a unique projective resolution up to a chain homotopy equivalence.

Example. Let $V=\mathbb{Z}$, be the trivial $\mathbb{Z} \Gamma$-module. Let $K$ be a cell complex which is $K(\Gamma, 1)$, i.e., $K$ is connected, $\pi_{1}(K) \cong \Gamma$ and $\pi_{i}(K)=0$ for $i \geq 2$. Let $X$ denote the universal cover of $K$. Lift the cell complex structure from $K$ to $X$. The group action $\Gamma \curvearrowright X$, determines a natural structure of a $\mathbb{Z} \Gamma$-module on the cellular chain complex $C_{*}(X)$. Since the latter is acyclic, we obtain a resolution of $\mathbb{Z}$ with

$$
P_{i}=C_{i}(X),
$$


and the homomorphism $P_{0} \rightarrow \mathbb{Z}$ given by the augmentation. Moreover, as the group $\Gamma$ acts freely on $X$, each module $P_{i}$ is a free $\mathbb{Z} \Gamma$-module:

$$
P_{i} \cong \oplus_{j \in C_{i}} \mathbb{Z} \Gamma,
$$

where $C_{i}$ is the set of $i$-cells in $K$.

A group $\Gamma$ is said to be of finite type, or $F P$ (over $R$ ), if there exists a resolution by finitely generated projective $R \Gamma$-modules

$$
0 \rightarrow P_{k} \rightarrow P_{k-1} \rightarrow \cdots \rightarrow P_{0} \rightarrow R \rightarrow 0 .
$$

For example, if there exists a finite cell complex $K=K(\Gamma, 1)$, then $\Gamma$ has finite type for an arbitrary ring $R$. Every group of finite type is finitely generated, although it does not have to be finitely-presented, see [24].

The cohomology of $\Gamma$ with coefficients in an $R \Gamma$-module $V, H^{*}(\Gamma, V)$, is defined as the homology of chain complex

$$
\operatorname{Hom}_{R \Gamma}\left(P_{*}, M\right),
$$

where $P_{*}$ is a projective resolution of the trivial $R \Gamma$-module $R$. The homology of $\Gamma$ with coefficients in $V, H_{*}(\Gamma, V)$, is the homology of the chain complex

$$
P_{*} \otimes_{R \Gamma} V .
$$

An example to keep in mind is the following. Suppose that $K$ is a manifold, or, more generally, a cell complex, which is an Eilenberg-MacLane space $K(\Gamma, 1)$. Then one can use the chain complex $C_{*}(X, R)$ as the resolution $P_{*}$. Therefore, for the trivial $\Gamma$-module $R$ we have

$$
H^{*}(\Gamma, R) \cong H^{*}(K, R), \quad H_{*}(\Gamma, R) \cong H_{*}(K, R) .
$$

For the more general modules $V$, in order to compute $H^{*}(\Gamma, V)$ and $H_{*}(\Gamma, V)$, one uses the (co)homology of $K$ with coefficients in an appropriate bundle over $K$.

Similarly, given a collection $\Pi$ of subgroups of $\Gamma$, one defines the relative (co)homology groups $H^{*}(\Gamma, \Pi ; V)$ and $H_{*}(\Gamma, \Pi ; V)$. Whenever discussing (co)homology with $R=\mathbb{Z}$, trivial $\mathbb{Z} \Gamma$-module, we will use the notation $H^{*}(\Gamma), H_{*}(\Gamma)$.

The (co)homology of groups behaves in a manner similar to the more familiar (co)homology of cell complexes. For instance, if $\Gamma$ admits an $n$-dimensional $K(\Gamma, 1)$, then $H^{i}(\Gamma, V)=H_{i}(\Gamma, V)=0$ for all $i>n$ and all $R \Gamma$-modules.

(Co)homological dimension. For a group $\Gamma$, let $c d_{R}(\Gamma)$ and $h d_{R}(\Gamma)$ denote the cohomological and homological dimensions of $\Gamma$ (over $R$ ):

$$
\begin{aligned}
& c d_{R}(\Gamma)=\sup \left\{n: \exists \text { an } R \Gamma \text {-module } V \text { so that } H^{n}(\Gamma, V) \neq 0\right\}, \\
& h d_{R}(\Gamma)=\sup \left\{n: \exists \text { an } R \Gamma \text {-module } V \text { so that } H_{n}(\Gamma, V) \neq 0\right\} .
\end{aligned}
$$

We will omit the subscript $\mathbb{Z}$ whenever $R=\mathbb{Z}$.

Using the relative (co)homology one defines the relative (co)homological dimension of $\Gamma$ with respect to a collection $\Pi$ of its subgroups, $c d_{R}(\Gamma, \Pi)$ and $h d_{R}(\Gamma, \Pi)$. 
We will use this definition in the case when $\Gamma$ is a Kleinian group as follows. Let $\mathcal{P}$ denote the set of all maximal (elementary) subgroups of $\Gamma$ which contain $\mathbb{Z}^{2}$. For every $\Gamma$-conjugacy class $\left[\Pi_{i}\right]$ in $\mathcal{P}$, choose a representative $\Pi_{i} \subset \Gamma$. Then $\Pi$ will denote the set of all these representatives $\Pi_{i}$. By abusing the notation, we will refer to the set $\Pi$ as the set of cusps of virtual rank $\geq 2$ in $\Gamma$.

If $\Gamma$ is of type $F P$, then

$$
h d_{R}(\Gamma)=c d_{R}(\Gamma), \quad \forall \operatorname{rings} R,
$$

see for instance [29]. In general,

$$
h d_{R}(\Gamma) \leq c d_{R}(\Gamma) \leq h d_{R}(\Gamma)+1 .
$$

Example. Let $\Gamma$ be a free group of finite rank $k>0$. Then $h d_{R}(\Gamma)=c d_{R}(\Gamma)$ for all rings $R$. Indeed, $\Gamma$ admits a finite $K(\Gamma, 1)$ which is the bouquet $B$ of $k$ circles. Since $B$ is 1-dimensional,

$$
h d_{R}(\Gamma)=c d_{R}(\Gamma) \leq 1 .
$$

On the other hand, by taking the trivial $R \Gamma$-module $V=R$ we obtain

$$
H_{1}(B, R)=R^{k},
$$

the direct sum of $k$ copies of $R$, and hence is nontrivial.

It turns out that the converse to this example is also true, which is an application of the famous theorem of J. Stallings on the ends of groups:

Theorem 4.1. (J. Stallings [205].) If $\Gamma$ is a finitely generated group with $c d(\Gamma)=1$, then $\Gamma$ is free.

This result was generalized by M. Dunwoody:

Theorem 4.2. (M. Dunwoody [66].) Let $R$ be an commutative ring with a unit.

1. If $\Gamma$ is a finitely generated torsion-free group with $c d_{R}(\Gamma)=1$, then $\Gamma$ is free.

2. If $\Gamma$ is finitely-presented and $c d_{R}(\Gamma)=1$ then $\Gamma$ is a free product of finite and cyclic groups with amalgamation over finite subgroups. In particular, $\Gamma$ is virtually free.

Duality groups. A group $\Gamma$ is said to be an $m$-dimensional duality group, if $\Gamma$ has type $F P$ and

$$
H^{i}(\Gamma, R \Gamma) \neq 0 \text {, for } i=m \text { and } H^{i}(\Gamma, R \Gamma)=0 \text {, for } i \neq m \text {. }
$$

For instance, a finitely-presented group $\Gamma$ is a 2-dimensional duality group (over $\mathbb{Z}$ ) if and only if $c d(\Gamma)=2$ and $\Gamma$ does not split as a nontrivial free product.

Poincaré duality groups. Poincaré duality groups are homological generalizations of the fundamental groups of closed aspherical manifolds.

Definition 4.3. A group $\Gamma$ is an (oriented) $m$-dimensional Poincaré duality group over $R$ (a $P D(m)$-group for short) if $\Gamma$ is of type $F P$ and

$$
H^{i}(\Gamma, R \Gamma) \cong R \text {, for } i=m \text { and } H^{i}(\Gamma, R \Gamma)=0 \text {, for } i \neq m \text {. }
$$


The basic examples are the fundamental groups of closed oriented aspherical $n$ manifolds.

This definition generalizes to (possibly non-oriented) $P D(n)$-groups, where we have to twist the module $V=R \Gamma$ by an appropriate orientation character $\chi: R \Gamma \rightarrow R$. The basic examples are the fundamental groups of closed aspherical $n$ manifolds $M$. The character $\chi$ in this case corresponds to the orientation character $\pi_{1}(M) \rightarrow R$.

We will need (in Section 5.3) the following relative version of the $P D(n)$ groups.

Definition 4.4. Let $\Gamma$ be an $(n-1)$-dimensional group of type $F P$, and let

$$
\Delta_{1}, \ldots, \Delta_{k} \subset \Gamma
$$

be $P D(n-1)$ subgroups of $\Gamma$. Set $\Delta:=\left\{\Delta_{1}, \ldots, \Delta_{k}\right\}$. Then, the group pair $(\Gamma, \Delta)$ is an $n$-dimensional Poincaré duality pair, or a $P D(n)$ pair, if the double of $\Gamma$ over the $\Delta_{i}$ 's is a $P D(n)$ group.

We recall that the double of $\Gamma$ over the $\Delta_{i}$ 's is the fundamental group of the graph of groups $\mathcal{G}$, where $\mathcal{G}$ has two vertices labelled by $\Gamma, k$ edges with the $i$-th edge labelled by $\Delta_{i}$, and edge monomorphisms are the inclusions $\Delta_{i} \rightarrow \Gamma$.

An alternate homological definition of $P D(n)$ pairs is the following: A group pair $(\Gamma, \Delta)$ is a $P D(n)$ pair if $\Gamma$ and each $\Delta_{i}$ has type $F P$, and

$$
H^{*}(\Gamma, \Delta ; \mathbb{Z} \Gamma) \simeq H_{c}^{*}\left(\mathbb{R}^{n}\right) .
$$

If $(\Gamma, \Delta)$ is a $P D(n)$ pair, where $\Gamma$ and each $\Delta_{i}$ admit a finite Eilenberg-MacLane space $X$ and $Y_{i}$ respectively, then the inclusions $\Delta_{i} \rightarrow \Gamma$ induce a map

$$
\sqcup_{i} Y_{i} \rightarrow X
$$

whose mapping cylinder $C$ gives a Poincaré pair $\left(C, \sqcup_{i} Y_{i}\right)$. The latter is a pair which satisfies Poincaré duality for manifolds with boundary with local coefficients, where $\sqcup_{i} Y_{i}$ serves as the boundary of $C$. The most important example of a $P D(n)$ pair is the following. Let $M$ be a compact manifold which is $K(\Gamma, 1)$. We suppose that the boundary of $M$ is the disjoint union

$$
\partial M=N_{1} \cup \cdots \cup N_{k},
$$

of $\pi_{1}$-injective components, each of which is a $K\left(\Delta_{i}, 1\right), i=1, \ldots, k$. Then the pair

$$
\left(\Gamma,\left\{\Delta_{1}, \ldots, \Delta_{k}\right\}\right)
$$

is a $P D(n)$ pair. See [30] for the details.

The following is one of the major problems in higher-dimensional topology:

Conjecture 4.5. (C.T.C. Wall, see a very detailed discussion in [131].) Suppose that $\Gamma$ is an finitely-presented $n$-dimensional Poincaré duality group over $\mathbb{Z}$. Then there exits a closed $n$-dimensional manifold $M$ which is $K(\Gamma, 1)$. 
This problem is open for all $n \geq 3$. The case $n=1$ is an easy corollary of the Stallings-Dunwoody theorem. In the case $n=2$, the positive solution is due to Eckmann, Linnel and Muller, see [67, 68]. This result was extended to the case of fields $R$ by B. Bowditch [41] and for general rings $R$ by M. Kapovich and B. Kleiner [123] and B. Kleiner [133]. Cannon's conjecture below is a special case (after Perelman's work) of Wall's problem for $n=3$ :

Conjecture 4.6 (J. Cannon). Suppose that $\Gamma$ is a Gromov-hyperbolic group whose ideal boundary is homeomorphic to $\mathbb{S}^{2}$. Then $\Gamma$ admits a cocompact properly discontinuous isometric action on $\mathbb{H}^{3}$.

\section{Topological rigidity and coarse compact core theorem}

First, few historical remarks. After the work of B. Maskit [156] and A. Marden [152], it became clear that the major developments in the 3-dimensional topology occurring at that time (in the 1960s and the early 1970s) were of extreme importance to the theory of Kleinian groups. The key topological results were:

1. Topological rigidity theorems of Stallings and Waldhausen. Under appropriate assumptions they proved that homotopy equivalence of Haken manifolds implies homeomorphism, see [95] for the detailed discussion. In the context of Kleinian groups, it meant that the (properly understood) algebraic structure of a geometrically finite Kleinian group $\Gamma \subset \operatorname{Mob}\left(\mathbb{S}^{2}\right)$ determines the topology of the associated hyperbolic 3 -manifold $\mathbb{H}^{3} / \Gamma$.

2. Dehn Lemma, Loop Theorem and their consequences. The most important (for Kleinian groups) of these consequences was the Scott compact core theorem [201, 202]. This theorem meant for (possibly geometrically infinite) Kleinian groups, that the hyperbolic 3 -manifold $M=\mathbb{H}^{3} / \Gamma$ admits a deformation retraction to an (essentially canonical) compact submanifold $M_{c} \subset M$ (the compact core of $M)$.

Remark 5.1. Of course, after W. Thurston entered the area of Kleinian groups, the theory experienced yet another radical change and became the theory of hyperbolic 3 -manifolds. However, this is another story.

We now turn to the higher dimensions.

\subsection{Results of Farrell and Jones}

The following conjecture is a natural generalization of the topological rigidity of 3-manifolds:

Conjecture 5.2 (A. Borel). Let $M, N$ be closed aspherical $n$-manifolds and $f$ : $M \rightarrow N$ is a homotopy-equivalence. Then $f$ is homotopic to a homeomorphism. (There is also a relative version of this conjecture.)

We refer the reader to [131] for a detailed discussion of Borel's Conjecture and its relation to Wall's Conjecture 4.5. Although, in full generality, Conjecture 
5.2 is expected to be false, in the last 20 years there has been a remarkable progress in proving this conjecture in the context to Kleinian groups. Most of these results appear in the works of T. Farrell and L. Jones. We collect some of them below.

Theorem 5.3. (T. Farrell and L. Jones [71].) Suppose that $\Gamma \subset \operatorname{Mob}\left(\mathbb{S}^{n}\right)$ is a convex-cocompact Kleinian group, $n \geq 4, N$ is a compact aspherical manifold (possibly with nonempty boundary $\partial N)$ and $f:\left(\bar{M}^{n+1}(\Gamma), M^{n}(\Gamma)\right) \rightarrow(N, \partial N)$ is a homotopy-equivalence which is a homeomorphism on the boundary. Then $f$ is homotopic to a homeomorphism (rel. $\left.M^{n}(\Gamma)\right)$.

Theorem 5.4. (T. Farrell and L. Jones, [72, Theorem 0.1]) Suppose that $X$ is a nonpositively curved closed Riemannian manifold, $Y$ is a closed aspherical manifold of dimension $\geq 5$ and $f: X \rightarrow Y$ is a homotopy-equivalence. Then $f$ is homotopic to a homeomorphism.

Theorem 5.5. (T. Farrell and L. Jones, [73, Proposition 0.10]) For each Kleinian group $\Gamma$ the Whitehead group $W h(\Gamma)$ is trivial.

By combining Theorem 5.5 with the $s$-cobordism theorem (see, e.g., [137, 186, 196]), one gets:

Corollary 5.6. Suppose that $W^{n+1}$ is a topological (resp. PL, smooth) h-cobordism so that $n \geq 4$ and $\pi_{1}\left(W^{n+1}\right)$ is isomorphic to a Kleinian group. Then $W$ is trivial in the topological (resp. PL, smooth) category.

\subsection{Limit sets and homological algebra}

Let $\Gamma \subset \operatorname{Mob}\left(\mathbb{S}^{n}\right)$ be a convex-cocompact subgroup with the limit set $\Lambda$ and $R$ be a ring. (One can also deal with geometrically finite groups by using relative cohomology, see [121].) The following theorem establishes a link between topology of the limit sets and the cohomology of $\Gamma$ :

Theorem 5.7. (M. Bestina, G. Mess [28].)

$$
H^{*}(\Gamma, R \Gamma) \cong H_{c}^{*}(H u l l(\Lambda), R) \cong \tilde{H}^{*-1}(\Lambda, R) .
$$

Here we are using the Chech cohomology of the limit set.

In particular, $\Gamma$ is an $m+1$-dimensional duality group over $\mathbb{Z}$ (see Section 4 ) iff $\tilde{H}^{*-1}(\Lambda)$ vanishes except in dimension $m$. In this case $\Lambda$, homologically, looks like an infinite bouquet of $m$-spheres. Moreover

$$
c d(\Gamma)=\operatorname{dim}(\Lambda),
$$

which gives a geometric interpretation of the cohomological dimension of $\Gamma$.

\subsection{Coarse compact core}

In this section we state the best (presently) available higher-dimensional generalization of the Scott compact core theorem. The main drawback of this result is that it applies only to Kleinian groups $\Gamma \subset \operatorname{Mob}\left(\mathbb{S}^{n}\right)$ which are $n$-dimensional duality groups. 
We first need some definitions. We recall that every end $e$ of a manifold $M$ admits a basic system of neighborhoods, which is a decreasing sequence $\left(E_{j}\right)$ of nested connected subsets of $M$ with compact frontier, so that

$$
\bigcap_{j \in \mathbb{N}} E_{j}=\emptyset \text {. }
$$

Given such $\left(E_{j}\right)$, we obtain the inverse system of the fundamental groups $\left(\pi_{1}\left(E_{j}\right)\right)$. Consider the image $\Gamma_{j}$ of $\pi_{1}\left(E_{j}\right)$ in $\pi_{1}(M)=\Gamma$. Then

$$
\Gamma_{1} \supset \Gamma_{2} \supset \cdots
$$

The fundamental group of $e$ (or, rather, its image in $\Gamma$ ) is defined as

$$
\pi_{1}(e)=\bigcap_{j \in \mathbb{N}} \Gamma_{j} .
$$

An end $e$ is called almost stable if every sequence $\left(\Gamma_{j}\right)$ as above is eventually constant, in which case

$$
\pi_{1}(e)=\Gamma_{j}
$$

for all sufficiently large $j$. (This notion is weaker than the notion of semistabe ends, which the reader might be familiar with.) For instance, if $M$ is an open handlebody of finite genus, then $M$ has unique end $e$, which is almost stable, whose fundamental group $\pi_{1}(e)$ is the free group $\pi_{1}(M)$. On the other hand, if $S$ is a surface of infinite genus with a unique end $e$, then $e$ is not almost stable. If $M$ is the complement to a Cantor set in $\mathbb{S}^{2}$, then no end of $M$ is almost stable.

The following is the Coarse Compact Core Theorem proved in [124] in the general context of coarse Poincaré duality spaces.

Theorem 5.8. (M. Kapovich, B. Kleiner [124].) Let $\Gamma \subset \mathbf{M o b}\left(\mathbb{S}^{\mathrm{n}}\right)$ be a Kleinian subgroup which is an $n$-dimensional duality group. Then the manifold $M=\mathbb{H}^{n+1} / \Gamma$ contains a compact submanifold $M_{c}$ (the coarse compact core) satisfying the following:

1. $\Gamma$ contains a finite collection $\Delta$ of $P D(n-1)$ subgroups $\Delta_{1}, \ldots, \Delta_{k}$.

2. The pair $(\Gamma, \Delta)$ is an $n$-dimensional Poincaré duality pair.

3. The group $\pi_{1}\left(M_{c}\right)$ maps onto $\pi_{1}(M)=\Gamma$.

4. The manifold $M$ has exactly $k$ ends $e_{1}, \ldots, e_{k}$, each of which is almost stable; the components $E_{1}, \ldots, E_{k}$ of $M \backslash M_{c}$ are basic neighborhoods of $e_{1}, \ldots, e_{k}$.

5. For every $i=1, \ldots, k, \pi_{1}\left(e_{i}\right)=\Delta_{i}$ is the image of $\pi_{1}\left(E_{i}\right)$ in $\Gamma$.

See Figure 1. In the case when $n=3$, this theorem, of course, is a special case of the Scott compact core theorem [201, 202]. More precisely, it covers the case when Scott compact core has incompressible boundary, for otherwise $\Gamma$ splits as a free product and is not a 2-dimensional duality group. If $M$ is a tame manifold, e.g., $\Gamma$ is geometrically finite, this theorem is also obvious. At the moment, all known examples of Kleinian groups in $\operatorname{Mob}\left(\mathbb{S}^{n}\right), n \geq 3$, which are $n$-dimensional duality groups, are geometrically finite. 


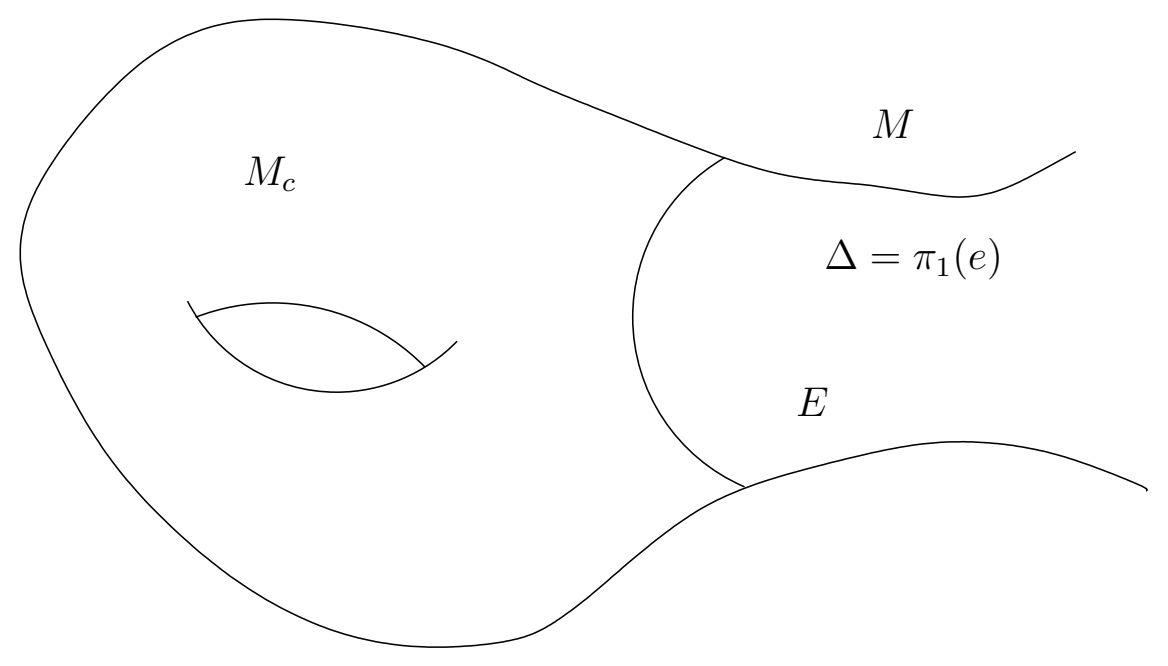

Figure 1. A coarse compact core.

Problem 5.9. Generalize Theorem 5.8 to groups of type FP which are not $n$-dimensional duality groups. (Of course, the conclusion of Part 1 of Theorem 5.8 would have to be suitably modified.)

\section{Notions of equivalence for Kleinian groups}

In this section we discuss various equivalence relations for Kleinian subgroups $\Gamma_{1}, \Gamma_{2}$ of $\operatorname{Mob}\left(\mathbb{S}^{n}\right)$. We start with the weakest one and end with the strongest.

(0) Algebraic: $\Gamma_{1}$ is isomorphic to $\Gamma_{2}$ as an abstract group.

(1) Dynamical: there exists a homeomorphism $f: \Lambda\left(\Gamma_{1}\right) \rightarrow \Lambda\left(\Gamma_{2}\right)$ such that $f \Gamma_{1} f^{-1}=\Gamma_{2}$; i.e., the groups $\Gamma_{1}$ and $\Gamma_{2}$ have the same topological dynamics on their limit sets. Thus, $\Gamma_{1}$ is geometrically finite iff $\Gamma_{2}$ is, since geometric finiteness can be stated in terms of topological dynamics of a group on its limit set (Theorem 2.5).

(2) Topological conjugation: there exists a homeomorphism $f: \mathbb{S}^{n} \rightarrow \mathbb{S}^{n}$ such that $f \Gamma_{1} f^{-1}=\Gamma_{2}$. (One can relax this by assuming that $f$ is defined only on the domain of discontinuity of $\Gamma_{1}$.)

(3) Quasiconformal conjugation: in (2) one can find a quasiconformal homeomorphism. In the case $n=1$ one should replace quasiconformal with quasisymmetric.

(4) Topological isotopy: in (2) there exists a continuous family of homeomorphisms $h_{t}: \mathbb{S}^{n} \rightarrow \mathbb{S}^{n}$ such that: $h_{0}=i d, \forall t, h_{t} \Gamma_{1} h_{t}^{-1} \subset \mathbf{M o b}\left(\mathbb{S}^{\mathrm{n}}\right)$ and $h_{1} \Gamma_{1} h_{1}^{-1}=\Gamma_{2}$. 
(5) Quasiconformal isotopy: in (4) all homeomorphisms are quasiconformal (quasisymmetric).

(6) Möbius conjugation: there is $f \in \operatorname{Mob}\left(\mathbb{S}^{n}\right)$ such that $f \Gamma_{1} f^{-1}=\Gamma_{2}$.

We refer the reader to [94, 99] for the definitions of quasisymmetric and quasiconformal homeomorphisms.

Below is a collection of facts about the relation between different notions of equivalence of Kleinian groups.

Suppose that both groups $\Gamma_{j}$ are geometrically finite and $\varphi: \Gamma_{1} \rightarrow \Gamma_{2}$ is an isomorphism which preserves the type of elements, i.e., for $\gamma \in \Gamma_{1}, \varphi(\gamma)$ is hyperbolic if and only if $\gamma$ is hyperbolic. It is clear that the above assumptions are necessary for getting the equivalence (1). The following theorem shows that these assumptions are also sufficient.

Theorem 6.1. (P. Tukia [217].) Under the above assumptions, the isomorphism $\varphi$ can be realized by the equivalence (1), i.e., there exists a (quasisymmetric) homeomorphism $f$ of the limit sets, so that $f \gamma f^{-1}=\varphi(\gamma)$ for all $\gamma \in \Gamma_{1}$. Moreover, if $f: \Omega\left(\Gamma_{1}\right) \rightarrow \Omega\left(\Gamma_{2}\right)$ is a $\varphi$-equivariant quasiconformal (quasisymmetric) homeomorphism, then $f$ admits a $\varphi$-equivariant quasiconformal (quasisymmetric) extension to the entire sphere.

Question 6.2. (Quasiconformal vs. topological.) Suppose that two Kleinian groups $\Gamma_{1}, \Gamma_{2} \subset \operatorname{Mob}\left(\mathbb{S}^{n}\right)$ are topologically conjugate by a homeomorphism $f$ (defined either on $\Omega\left(\Gamma_{1}\right)$, or on $\Lambda\left(\Gamma_{1}\right)$, or on the entire $\left.\mathbb{S}^{n}\right)$, which induces a type-preserving isomorphism $\varphi: \Gamma_{1} \rightarrow \Gamma_{2}$. Does it imply that $\varphi$ is induced by a quasiconformal (quasisymmetric) homeomorphism with the same domain as $f$ ?

Note that, for every $n$, the above question actually consists of 3 subquestions, depending on the domain of $f$. Here is what is currently known about these questions:

1. If $n=1$ then all three questions have the affirmative answer and the proof is rather elementary. It also follows for instance from Theorem 6.1.

2. If $n=2$ then the answer to all three questions is again positive, but the proof is highly nontrivial. The easiest case is when the homeomorphism $f$ is defined on $\Omega\left(\Gamma_{1}\right)$. Then we get the induced homeomorphism $\bar{f}$ of the quotient surfaces $S_{1} \rightarrow S_{2}$, where $S_{i}=\Omega\left(\Gamma_{i}\right) / \Gamma_{i}$. The existence of a diffeomorphism $S_{1} \rightarrow S_{2}$ homotopic to $\bar{f}$ follows from the uniqueness of the smooth structure on surfaces. If $S_{1}$ is compact, then this diffeomorphism lifts to an equivariant quasiconformal homeomorphism $\Omega\left(\Gamma_{1}\right) \rightarrow \Omega\left(\Gamma_{2}\right)$. Two noncompact surfaces can be diffeomorphic but not quasiconformally homeomorphic: For instance, the open disk is not quasiconformally equivalent to the complex plane. However, since $\varphi$ is type-preserving, Ahlfors Finiteness Theorem [3] in conjunction with a lemma of Bers and Maskit (see, e.g., [116, Corollary 4.85]), implies the existence of a quasiconformal homeomorphism $S_{1} \rightarrow S_{2}$.

If $f$ is defined on the limit set and $\Gamma_{1}, \Gamma_{2}$ are geometrically finite, then the positive answer is a special case of Tukia's theorem 6.1. However, if the groups 
$\Gamma_{i}$ are not geometrically finite, the proof becomes very difficult and is a corollary of the solution of the Ending Lamination Conjecture in the work of J. Brock, R. Canary and Y. Minsky in [46, 166, 167], and M. Rees [193].

Combination of the Ahlfors Finiteness Theorem with the Ending Lamination Conjecture also gives the positive answer in the case when $f$ is defined on $\mathbb{S}^{2}$.

3. If $f$ is defined on $\Omega\left(\Gamma_{1}\right)$, then the answer is positive provided that $n \neq 4$ and $M^{n}\left(\Gamma_{1}\right)$ is compact. This is a consequence of the theorem of D. Sullivan [207], who proved uniqueness of the quasiconformal structure on compact $n$-manifolds $(n \neq 4)$ : Apply Sullivan's theorem to the manifolds $M^{n}\left(\Gamma_{i}\right), i=1,2$, and lift the quasiconformal homeomorphism to the domain of discontinuity.

Remark 6.3. An alternative proof of Sullivan's theorem and its generalization was given by J. Luukkainen in [147], see also [220].

If $n=4, f$ is defined on $\Omega\left(\Gamma_{1}\right)$, and $M^{4}\left(\Gamma_{1}\right)$ is compact, then the situation is unclear but one probably should expect the negative answer since the uniqueness of quasiconformal structures in dimension 4 was disproved by S. Donaldson and D. Sullivan [64].

Question 6.4. Is there a pair of Kleinian groups $\Gamma_{1}, \Gamma_{2} \subset \operatorname{Mob}\left(\mathbb{S}^{n}\right)$ so that the manifolds $M^{n}\left(\Gamma_{1}\right), M^{n}\left(\Gamma_{2}\right)$ are homeomorphic but not diffeomorphic?

Note that in view of the examples in [70], the positive answer to the above question would not be too surprising.

If $f$ is defined on $\Omega\left(\Gamma_{1}\right)$ and we do not assume compactness of $M^{n}\left(\Gamma_{1}\right)$, then the answer to Question 6.2 is negative in a variety of ways.

(a) For instance, take singly degenerate groups $\Gamma_{1}, \Gamma_{2} \subset \operatorname{Mob}\left(\mathbb{S}^{2}\right)$, which are both isomorphic to the fundamental group of a closed oriented surface $S$, contain no parabolic elements and have distinct ending laminations. Then $\Omega\left(\Gamma_{i}\right) \subset \mathbb{S}^{2}$ are open disks $D_{i}$ for both $i$. There exists an equivariant homeomorphism $h: D_{1} \rightarrow D_{2}$, which induces an isomorphism $\varphi: \Gamma_{1} \rightarrow \Gamma_{2}$. However, since the ending laminations are different, there is no equivariant homeomorphism $\Lambda\left(\Gamma_{1}\right) \rightarrow \Lambda\left(\Gamma_{2}\right)$.

Now extend both groups to the 3 -sphere so that $\Gamma_{i} \subset \operatorname{Mob}\left(\mathbb{S}^{3}\right), i=1,2$. Then the 3-dimensional domains of discontinuity $B_{i}$ of both groups are diffeomorphic to the open 3-ball, $i=1,2$; the quotient manifolds are

$$
M^{3}\left(\Gamma_{i}\right)=B_{i} / \Gamma_{i} \cong S \times \mathbb{R}, \quad i=1,2 .
$$

Therefore there exists an equivariant diffeomorphism $f: B_{1} \rightarrow B_{2}$. We claim that this map cannot be quasiconformal. Indeed, otherwise it would extend to an equivariant homeomorphism of the limit sets (which are planar subsets of $\mathbb{R}^{3}$ ). This is a contradiction.

(b) One can construct geometrically finite examples as well. The reason is that even though all (orientation-preserving) parabolic elements of $\operatorname{Mob}\left(\mathbb{S}^{2}\right)$ are quasiconformally conjugate, the analogous assertion is false for the parabolic elements of $\operatorname{Mob}\left(\mathbb{S}^{3}\right)$. Suppose that $\tau$ is the translation in $\mathbb{R}^{3}$ by a nonzero vector $v$. 
Let $R_{\theta_{i}}, i=1,2$, denote the rotations around $v$ by the angles $\theta_{1}, \theta_{2} \in[0, \pi]$. Then the skew motions

$$
\gamma_{i}=R_{\theta_{i}} \circ \tau_{i}, \quad i=1,2
$$

are parabolic elements of $\mathbf{M o b}\left(\mathbb{S}^{3}\right)$. One can show that

Proposition 6.5. The Möbius transformations $\gamma_{1}$ and $\gamma_{2}$ are quasiconformally conjugate in $\mathbb{S}^{3}$ if and only if $\theta_{1}=\theta_{2}$.

The proof is based on a calculation of the extremal length of a certain family of curves in $\mathbb{R}^{3}$ and we will not present it here.

Note that the cyclic groups $\Gamma_{i}=\left\langle\gamma_{i}\right\rangle$ are geometrically finite, the isomorphism $\varphi: \Gamma_{1} \rightarrow \Gamma_{2}$ sending $\gamma_{1}$ to $\gamma_{2}$ is type-preserving. The quotient manifolds $M^{3}\left(\Gamma_{i}\right)$ are both diffeomorphic to $\mathbb{R}^{2} \times \mathbb{S}^{1}$, therefore there exists a $\varphi$-equivariant diffeomorphism $f: \Omega\left(\Gamma_{1}\right) \rightarrow \Omega\left(\Gamma_{2}\right)$ which, of course, extends to a homeomorphism $\mathbb{S}^{3} \rightarrow \mathbb{S}^{3}$. However, according to Proposition 6.5 , this homeomorphism cannot be made quasiconformal.

These examples do not resolve the following:

Question 6.6. Suppose that $\Gamma_{1}, \Gamma_{2} \subset \mathbf{M o b}\left(\mathbb{S}^{n}\right), n \geq 3$, are Kleinian groups and $f: \Lambda\left(\Gamma_{1}\right) \rightarrow \Lambda\left(\Gamma_{2}\right)$ is a homeomorphism which induces an isomorphism $\Gamma_{1} \rightarrow \Gamma_{2}$. Does it follow that $f$ is quasisymmetric?

If $n \leq 2$, then (in the list of equivalences between Kleinian groups) we have the implication

$$
(3) \Rightarrow(5) \text {. }
$$

Indeed, consider a quasiconformal homeomorphism $f$ conjugating Kleinian groups $\Gamma_{1}$ and $\Gamma_{2}$ and let $\mu$ denote the Beltrami differential of $f$. Then for $t \in[0,1]$ the solutions of the Beltrami equation

$$
\frac{\partial f_{t}}{\partial \bar{z}}=t \mu \frac{\partial f}{\partial z}
$$

also conjugate $\Gamma_{1}$ to Kleinian subgroups of $\operatorname{Mob}\left(\mathbb{S}^{2}\right)$, see, e.g., [20]. This gives the required quasiconformal isotopy. Since (2) is equivalent to (3) for $n \leq 2$, it follows that for $n \leq 2$ we have

$$
(2) \Longleftrightarrow(3) \Longleftrightarrow(4) \Longleftrightarrow(5)
$$

This argument however fails completely in higher dimensions, since the Beltrami equation in $\mathbb{R}^{n}$ for $n \geq 3$ is overdetermined.

Question 6.7. In the list of equivalences between Kleinian groups:

(a) Does $(2) \Rightarrow(4)$ ?

(b) Does $(3) \Rightarrow(5)$ ?

One can show (using quasiconformal stability, see Section 11.2, cf. [154, Theorem 7.2]) that for convex-cocompact groups parts (a) and (b) of the above question are equivalent. In Theorem 11.11 we give examples of convex-cocompact Kleinian groups in $\operatorname{Mob}\left(\mathbb{S}^{n}\right), n \geq 5$, for which the answer to Question 6.7 is negative. The 
situation in dimensions 3 and 4 at the moment is unclear, but we expect in these dimensions the answer to be negative as well.

The implications $(\mathrm{i}) \Rightarrow(6)$ for $\mathrm{i} \leq 5$ are, of course, extremely rare. The most celebrated example is provided by the Mostow rigidity theorem:

Theorem 6.8. Suppose that $\Gamma_{1}, \Gamma_{2} \subset \operatorname{Mob}\left(\mathbb{S}^{n}\right)$ are lattices and $n \geq 2$. Then $(0) \Rightarrow$ (6) for these groups.

See [175] for G. Mostow's original proof or [120] for a more elementary argument along the same lines which uses only the analytical properties of quasiconformal mappings. A completely different argument due to M. Gromov can be found in [18]. Yet another proof is an application of the barycentric maps [21]. Note that, presently, there are no proofs using equivariant harmonic maps.

Mostow's ergodic arguments were greatly generalized by D. Sullivan in [208], see also $[5]$ :

Theorem 6.9. (D. Sullivan [208].) Suppose that $\Gamma_{1}, \Gamma_{2} \subset \mathbf{M o b}\left(\mathbb{S}^{n}\right)$ are Kleinian groups whose limit set is the entire $\mathbb{S}^{n}$ and so that the action of $\Gamma_{1}$ on $\mathbb{S}^{n}$ is recurrent. Then $(3) \Rightarrow(6)$ for these groups.

The action of $\Gamma \subset \operatorname{Mob}\left(\mathbb{S}^{n}\right)$ on $\mathbb{S}^{n}$ is called recurrent if for every measurable subset $E \subset \mathbb{S}^{n}$ of positive Lebesgue measure, the measure of the intersection $\gamma(E) \cap E$ is positive for some $\gamma \in \Gamma \backslash\{1\}$.

\section{Groups with zero-dimensional limit sets}

In what follows, we let dim denote the covering dimension of topological spaces, see for instance [96]. Suppose that $\Gamma \subset \operatorname{Mob}\left(\mathbb{S}^{n}\right)$ is a non-elementary Kleinian subgroup of $\mathbf{M o b}\left(\mathbb{S}^{n}\right)$ and $\operatorname{dim}(\Lambda(\Gamma))=0$; hence $\Lambda(\Gamma)$ is totally disconnected (its only connected components are points). Recall that a discontinuum is a nonempty perfect totally disconnected compact topological space, see, e.g., [8]. Hence $\Lambda(\Gamma)$ is a discontinuum. It follows (see, e.g., [8]) that $\Lambda(\Gamma)$ is homeomorphic to the standard Cantor set $K \subset[0,1]$. Below is a couple of examples of Kleinian groups whose limit sets are totally disconnected.

Example. (A Schottky group, see, e.g., $[138,157]$.) Let $n, k \geq 1$. Suppose that we are given a collection of disjoint closed topological $n$-balls

$$
B_{1}^{+}, \ldots, B_{k}^{+}, B_{1}^{-}, \ldots, B_{k}^{-} \subset \mathbb{S}^{n}
$$

and Möbius transformations $\gamma_{j} \in \mathbf{M o b}\left(\mathbb{S}^{n}\right)$ so that $\gamma_{j}\left(\operatorname{int}\left(B_{j}^{+}\right)\right)=\operatorname{ext}\left(B_{j}^{-}\right)$. We assume that for each pair $B_{j}^{+}, B_{j}^{-}$there exists a diffeomorphism of $\mathbb{S}^{n}$ which carries 
these balls to the round balls. ${ }^{1}$ Then

$$
\Phi:=\mathbb{S}^{n}-\bigcup_{j=1}^{k}\left(B_{j}^{+} \cup \operatorname{int}\left(B_{j}^{-}\right)\right)
$$

is a fundamental domain for the group $\Gamma$ generated by $\gamma_{1}, \ldots, \gamma_{k}$. The group $\Gamma$ is called a Schottky group. It is isomorphic to a free group of rank $k$, and the limit set of $\Gamma$ is a discontinuum provided that $k \geq 2$. Every nontrivial element of $\Gamma$ is hyperbolic.

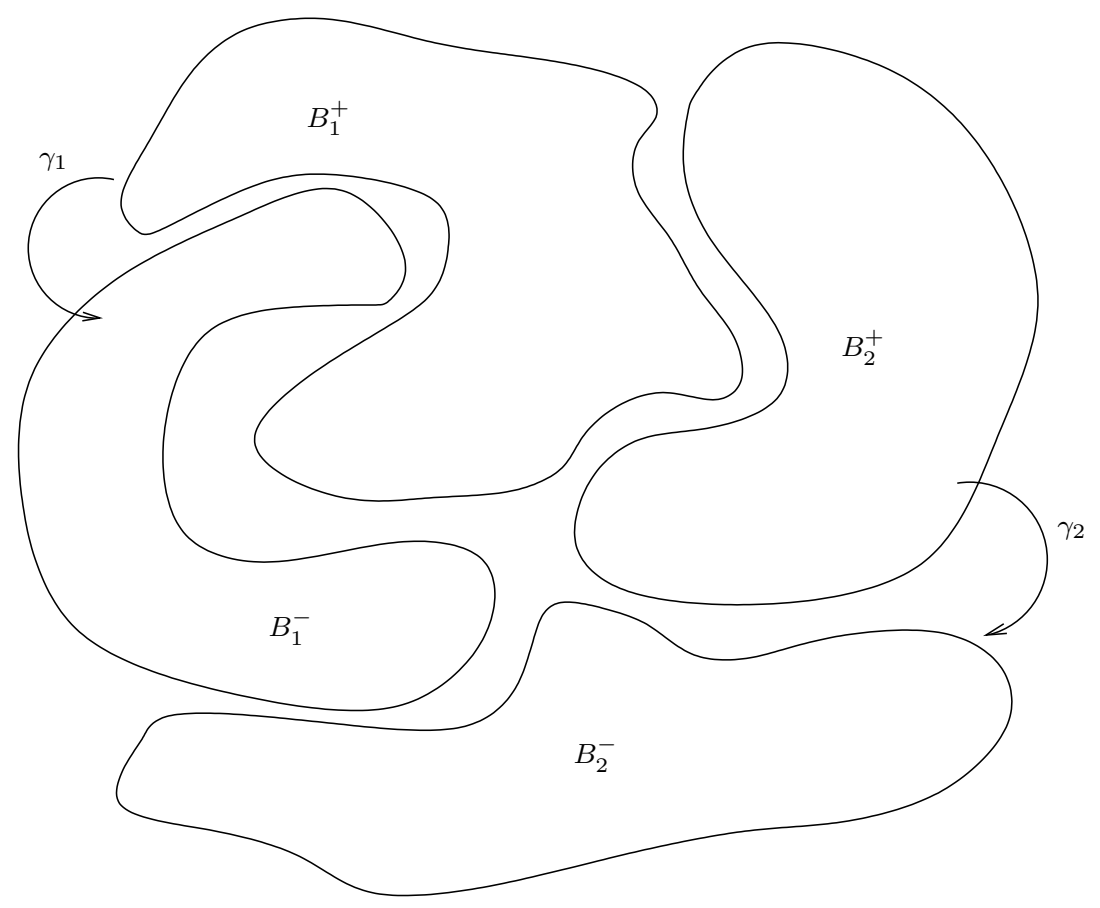

FiguRE 2. A Schottky group.

Before giving the next example we need a definition. Suppose that $\Gamma \subset$ $\operatorname{Mob}\left(\mathbb{S}^{n}\right)$ is a nontrivial elementary subgroup. Then, after conjugating $\Gamma$ if necessary, we can assume that either:

1. $\Gamma$ fixes $0, \infty \in \overline{\mathbb{R}^{n}}=\mathbb{S}^{n}$ and therefore is generated by $\gamma(x)=A x$, where $A$ is the product of a scalar $c>1$ by an orthogonal matrix.

2. Or $\Gamma$ acts on $\mathbb{R}^{n} \subset \mathbb{S}^{n}$ by Euclidean isometries.

\footnotetext{
${ }^{1}$ By the smooth Schoenflies theorem, for $n \neq 4$ it suffices to assume that the balls $B_{j}^{+}$have smooth boundary.
} 
In the first case we take the fundamental domain $\Phi$ for the action of $\Gamma$ on $\mathbb{S}^{n}$ to be an annulus bounded by two disjoint round spheres. In the second case we take a Dirichlet fundamental domain $\Phi \subset \mathbb{R}^{n}$ for $\Gamma$.

We refer to the fundamental domains $\Phi$ as standard fundamental domains. A fundamental domain for $\Gamma$ is topologically standard if it is the image of a standard fundamental domain of $\Gamma$ under a diffeomorphism of $\Omega(\Gamma)$ commuting with $\Gamma$. For instance, the fundamental domain for a rank 1 Schottky group is topologically standard. Therefore, the fundamental domain $\Phi$ for the Schottky group satisfies the property that it is the intersection of topologically standard fundamental domains

$$
\Phi_{j}=\mathbb{S}^{n}-\left(B_{j}^{+} \cup \operatorname{int}\left(B_{j}^{-}\right)\right)
$$

for the groups $\Gamma_{j}=\left\langle\gamma_{j}\right\rangle$.

Given a domain $\Phi \subset \mathbb{S}^{n}$, we let $\Phi^{c} \subset \mathbb{S}^{n}$ denote the closure of the complement of $\Phi$. We are now ready for the second example which is a generalization of the first.

Example. (Schottky-type groups, see, e.g., $[138,157]$.) Start with a collection of elementary Kleinian groups $\Gamma_{i} \subset \operatorname{Mob}\left(\mathbb{S}^{n}\right), i=1, \ldots, k$. Let $\Phi_{i} \subset \mathbb{S}^{n}$ be topologically standard fundamental domains for these groups. Assume that

$$
\Phi_{i}^{c} \cap \Phi_{j}^{c}=\emptyset
$$

for all $i \neq j$. Let $\Gamma \subset \operatorname{Mob}\left(\mathbb{S}^{n}\right)$ be the group generated by $\Gamma_{1}, \ldots, \Gamma_{k}$. Then:

1. As an abstract group, $\Gamma$ is isomorphic to the free product $\Gamma_{1} * \cdots * \Gamma_{k}$.

2. $\Phi:=\Phi_{1} \cap \cdots \cap \Phi_{k}$ is a fundamental domain for the group $\Gamma$.

3. The limit set of $\Gamma$ is totally disconnected.

The groups $\Gamma$ obtained in this fashion are called Schottky-type groups.

A Schottky-type group is called classical if it admits a fundamental domain $\Phi:=\Phi_{1} \cap \ldots \cap \Phi_{k}$, so that each $\Phi_{i}$ is geometrically standard. It is not difficult to see that Schottky-type groups are geometrically finite. For instance, consider the case of a Schottky group $\Gamma$ of rank $k$, for $n \geq 2$. We have the map

$$
j: \mathbb{Z}=H_{n}\left(M^{n}(\Gamma)\right) \rightarrow H_{n}\left(\bar{M}^{n+1}(\Gamma)\right)
$$

induced by the inclusion of manifolds. Since the manifold $\bar{M}=\bar{M}^{n+1}(\Gamma)$ is $K(\Gamma, 1)$, it follows that

$$
H^{n}(\bar{M})=H^{n}(\Gamma)=H^{n}(B)=0,
$$

where $B$ is the bouquet of $k$ circles. Therefore $j=0$. Hence $\bar{M}$ is compact and hence $\Gamma$ is convex-cocompact. A similar argument works for Schottky-type groups, provided that one uses cohomology relative to the cusps.

The quotient manifolds of the Schottky-type groups $\Gamma$ have a rather simple topology, as it follows from the explicit description of their fundamental domains. Namely, let $M_{i}=M^{n}\left(\Gamma_{i}\right), i=1, \ldots, k$. Then we get the smooth connected sum decomposition

$$
M^{n}(\Gamma)=M_{1} \# \cdots \# M_{k} .
$$

By combining this with Theorem 6.1 we obtain 
$\Phi_{2}^{c}$

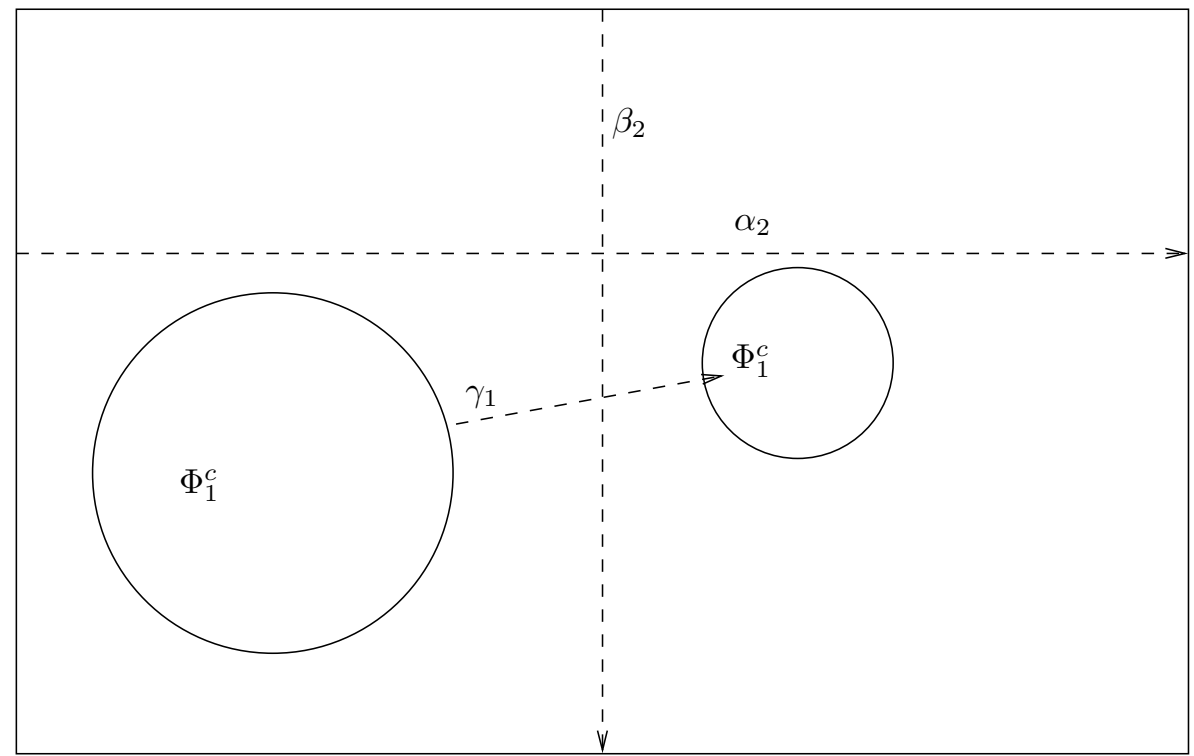

Figure 3. A classical Schottky-type group isomorphic to $\mathbb{Z} * \mathbb{Z}^{2}$ with $\mathbb{Z}=\left\langle\gamma_{1}\right\rangle, \mathbb{Z}^{2}=\left\langle\alpha_{2}, \beta_{2}\right\rangle$.

Proposition 7.1. 1. Suppose that $\Gamma, \Gamma^{\prime}$ are Schottky groups of the same rank. Then there exists a quasiconformal homeomorphism $f: \mathbb{S}^{n} \rightarrow \mathbb{S}^{n}$ which conjugates $\Gamma$ to $\Gamma^{\prime}$, i.e., $f \Gamma f^{-1}=\Gamma^{\prime}$.

2. Suppose that $\Gamma, \Gamma^{\prime}$ are Schottky-type groups and $\varphi: \Gamma \rightarrow \Gamma^{\prime}$ is a typepreserving isomorphism, so that for every free factor $\Gamma_{i}$ in $\Gamma$, the restriction $\varphi: \Gamma_{i} \rightarrow \Gamma_{i}^{\prime} \subset \Gamma^{\prime}$ is induced by a quasiconformal homeomorphism of $\mathbb{S}^{n}$. Then there exists a quasiconformal homeomorphism $f: \mathbb{S}^{n} \rightarrow \mathbb{S}^{n}$ which induces the isomorphism $\varphi$.

Question 7.2. Let $n \geq 3$. Is there a quasiconformal isotopy between $\Gamma$ and $\Gamma^{\prime}$ in the above theorem (either part 1 or part 2)?

In the case when $\Gamma$ and $\Gamma^{\prime}$ are both classical, the positive answer follows rather easily. In the non-classical case the above question is open even if $n=3$ and $\Gamma$ is a Schottky group.

Schottky subgroups of $\operatorname{Mob}\left(\mathbb{S}^{2}\right)$ can be characterized as follows:

Theorem 7.3. (B. Maskit [155].) A Kleinian subgroup $\Gamma \subset \mathbf{M o b}\left(\mathbb{S}^{2}\right)$ is a Schottky group if and only if $\Gamma$ is free, has nonempty domain of discontinuity in $\mathbb{S}^{2}$ and consists only of hyperbolic elements. 
This result was generalized by N. Gusevskii and N. Zindinova [92]:

Theorem 7.4. Let $\Gamma \subset \operatorname{Mob}\left(\mathbb{S}^{2}\right)$ be a Kleinian subgroup, which has nonempty domain of discontinuity in $\mathbb{S}^{2}$ and is isomorphic to a Schottky-type group $\Gamma^{\prime}$ via a type-preserving isomorphism $\Gamma \rightarrow \Gamma^{\prime}$. Then $\Gamma$ is a Schottky-type group.

Both theorems are easy under the assumption that $\Gamma$ is geometrically finite, the key point here is that (in dimension 2) one can prove geometric finiteness under the above mild assumptions.

If $\Gamma$ is a Kleinian subgroup of $\operatorname{Mob}\left(\mathbb{S}^{3}\right)$, then the above results are not longer true, moreover, $\Gamma$ can be geometrically infinite. For instance, take a free finitely generated purely hyperbolic discrete subgroup of $\operatorname{PSL}(2, \mathbb{C})$, whose limit set is the 2 -sphere (the existence of such groups was first established by V. Chuckrow [56]). The Möbius extension of this group to $\mathbb{S}^{3}$ has nonempty domain of discontinuity, but is not geometrically finite.

Tameness of limit sets. Below we address the following:

Question 7.5. Suppose that $\Gamma \subset \operatorname{Mob}\left(\mathbb{S}^{n}\right)$ is a Kleinian group, whose limit set is a discontinuum. What can be said about the embedding $\Lambda(\Gamma) \subset \mathbb{S}^{n}$ ?

A discontinuum $D \subset \mathbb{S}^{n}$ is called tame if there exists a homeomorphism $f: \mathbb{S}^{n} \rightarrow \mathbb{S}^{n}$ which carries $D$ to the Cantor set $K \subset[0,1]$ and is called wild otherwise. It is a classical (and easy) result that every discontinuum in $\mathbb{S}^{2}$ is tame, see, e.g., [31]. The (historically) first example of a wild discontinuum was the Antoine's necklace $A \subset \mathbb{S}^{3}$ :

$$
\pi_{1}\left(\mathbb{S}^{3} \backslash A\right) \neq\{1\},
$$

which explains why $A$ is wild, see [31]. D. DeGryse and R. Osborne [61] constructed for every $n \geq 3$ examples of wild discontinua $D_{n} \subset \mathbb{S}^{n}$, such that

$$
\pi_{1}\left(\mathbb{S}^{n} \backslash D_{n}\right)=\{1\} .
$$

See also [77] for infinitely many inequivalent 3-dimensional examples of this type.

The algebraic structure of Kleinian groups with totally disconnected limit sets is given by

Theorem 7.6. (R. Kulkarni [141].) Suppose that a Kleinian group $\Gamma \subset \mathbf{M o b}\left(\mathbb{S}^{\mathrm{n}}\right)$ has a totally disconnected limit set. Then $\Gamma$ is isomorphic to a Schottky-type group.

One can even describe (to some extent) fundamental domains of such groups:

Theorem 7.7. (N. Gusevskii [90].) Suppose that the limit set of $\Gamma \subset \operatorname{Mob}\left(\mathbb{S}^{n}\right)$ is totally disconnected. Then $\Gamma$ admits a fundamental domain $\Phi$ of the same shape as in Example 7, only the fundamental domains $\Phi_{i}$ for $\Gamma_{i}$ 's are not required to be topologically standard.

The proof of Theorem 7.7 is based on the following 
Theorem 7.8. (M.Brin [45].) Let $\tilde{M}$ be a smooth oriented $n$-manifold of dimension $>2$, so that $H^{1}(\tilde{M})=0$. Let $\Gamma \curvearrowright \tilde{M}$ is a smooth properly discontinuous free action.

Then, for every smooth oriented compact hypersurface $\Sigma$ in $\tilde{M}$ and an open neighborhood $U$ of $\Gamma \cdot \Sigma$, there exists a smooth compact connected oriented hypersurface $\Sigma^{*} \subset U$ such that for every $\gamma \in \Gamma$ either $\gamma \Sigma^{*} \cap \Sigma^{*}=\emptyset$ or $\gamma \Sigma^{*}=\Sigma^{*}$.

This theorem allows one to split (inductively) the Kleinian group $\Gamma$ as a free product in a "geometric fashion": Start with a compact hypersurface in $\Omega(\Gamma)$ which separates components of $\Lambda(\Gamma)$. Find $\Sigma^{*}$ as in Brin's theorem which still separates. Then cut open the manifold $M^{n}(\Gamma)$ along the projection of $\Sigma^{*}$. This decomposition yields a free product decomposition $\Gamma=\Gamma^{\prime} * \Gamma^{\prime \prime}$ so that $\Gamma$ is a Klein combination of the groups $\Gamma^{\prime}, \Gamma^{\prime \prime}$. Continue inductively. Finite generation of $\Gamma$ implies that the decomposition process will terminate and the terminal groups must be elementary. Note that if all $\Sigma^{*}$ were spheres, then this decomposition would be of Schottky-type.

Corollary 7.9. Every Kleinian group with a totally disconnected limit set is geometrically finite.

Proof. Repeat the arguments which we used to establish geometric finiteness of Schottky groups.

Problem 7.10. Suppose that $\Gamma \subset \operatorname{Mob}\left(\mathbb{S}^{n}\right)$ is such that $\Lambda(\Gamma)$ is a tame discontinuum. Does is follow that $\Gamma$ is a Schottky-type group?

If $n=2$ then the affirmative answer to this question follows for instance from Maskit's theorem. If $n=3$ then the answer is again positive; moreover,

Proposition 7.11. Suppose that $\Gamma \subset \operatorname{Mob}\left(\mathbb{S}^{3}\right)$ is such that $\Lambda(\Gamma)$ is totally disconnected and $\pi_{1}(\Omega(\Gamma))=1$. Then $\Gamma$ is a Schottky-type group

Proof. Under the above assumptions, $\pi_{2}(\Omega(\Gamma)) \neq\{0\}$; hence, by the Sphere Theorem (see, e.g., [95]), we can find a smooth hypersurface $\Sigma^{*}$ as in Brin's theorem, so that $\Sigma^{*}$ is diffeomorphic to $\mathbb{S}^{2}$. Therefore, as we saw above, it follows that $\Gamma$ is a Schottky-type group.

This argument however fails for $n \geq 4$, where Problem 7.10 is still open. On the other hand, there are Kleinian subgroups of $\operatorname{Mob}\left(\mathbb{S}^{3}\right)$ with wild discontinua as limit sets. The first such example was given by M. Bestvina and D. Cooper:

Theorem 7.12. (M. Bestvina, D. Cooper [25].) There exists a Kleinian group $\Gamma \subset$ $\operatorname{Mob}\left(\mathbb{S}^{3}\right)$ which contains parabolic elements, so that $\Lambda(\Gamma)$ is a wild discontinuum.

The proof that $\pi_{1}(\Omega(\Gamma)) \neq 1$ presented in [25] was incomplete; however the gap was filled several years later by S. Matsumoto:

Theorem 7.13. (S. Matsumoto [158, 159], see also [91].) There are Kleinian groups $\Gamma$ in $\operatorname{Mob}\left(\mathbb{S}^{3}\right)$ without parabolic elements whose limit sets are wild discontinua. 


\section{Groups with one-dimensional limit sets}

The simplest examples of 1-dimensional limit sets of Kleinian groups are topological circles. For instance, the limit set of a lattice $\Gamma \subset \operatorname{Isom}\left(\mathbb{H}^{2}\right)$ is the round circle. Of course, even if the limit set of $\Gamma \subset \operatorname{Mob}\left(\mathbb{S}^{n}\right)$ is a topological circle, its embedding in $\mathbb{S}^{n}$ can be complicated. We will discuss this issue later on. For now, we are only interested in the topology of the limit set itself.

Given convex-cocompact Kleinian groups $\Gamma_{1}, \Gamma_{2} \subset \mathbf{M o b}\left(\mathbb{S}^{n}\right)$ with 1-dimensional limit sets, one can use Klein-Maskit Combination theorems in order to get convex-cocompact Kleinian groups $\Gamma \subset \operatorname{Mob}\left(\mathbb{S}^{\mathrm{n}}\right)$ isomorphic to

$$
\Gamma_{1} *_{\Delta} \Gamma_{2},
$$

where $\Delta$ is either trivial or infinite cyclic. The limit sets of the resulting groups are again 1-dimensional. For instance, if $\Lambda\left(\Gamma_{i}\right)$ is a topological circle for $i=1,2$ then the limit set of $\Gamma=\Gamma_{1} * \Gamma_{2}$ will be disconnected: The connected components of $\Lambda(\Gamma)$ are topological circles and points. Similarly, if $\Delta=\mathbb{Z}$, then the limit set of $\Gamma=\Gamma_{1} *_{\Delta} \Gamma_{2}$ will have cut pairs: The complement to the 2 -point set $\Lambda(\Delta)$ in $\Lambda(\Gamma)$ is disconnected.

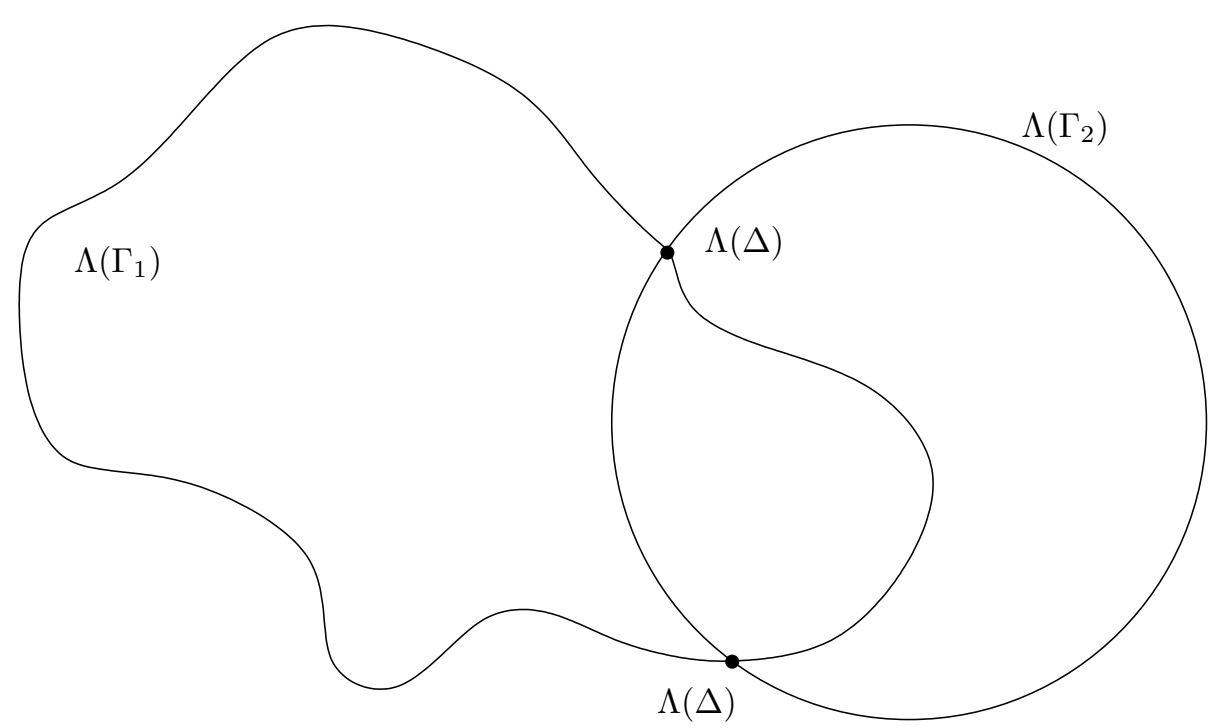

FiguRE 4. Combination of two 1-quasifuchsian groups: $\Gamma=\Gamma_{1} *_{\mathbb{Z}} \Gamma_{2}$.

These constructions are, of course, not very illuminating. Therefore we are interested in examples of 1-dimensional limit sets which are connected and which do not contain cut-pairs. It turns out that there are only two such examples: 
1. The Sierpinski carpet $\mathcal{S}$. Start with the unit square $S=I \times I$. Subdivide this square into 9 squares of the size $\frac{1}{3} \times \frac{1}{3}$ and then remove from $S$ the open middle square $\left(\frac{1}{3}, \frac{2}{3}\right) \times\left(\frac{1}{3}, \frac{2}{3}\right)$. Repeat this for each of the remaining $\frac{1}{3} \times \frac{1}{3}$ sub-squares in $S$ and continue inductively. After removing a countable collection of open squares we are left with a compact subset $\mathcal{S} \subset \mathbb{R}^{2}$, called the Sierpinski carpet.

2. The Menger curve $\mathcal{M}$. Start with the unit cube $Q=I \times I \times I$. Each face $F_{i}$ of $Q$ contains a copy of the Sierpinski carpet $\mathcal{S}_{i}$. Let $p_{i}: Q \rightarrow F_{i}$ denote the orthogonal projection. Then

$$
\mathcal{M}:=\bigcap_{i} p_{i}^{-1}\left(\mathcal{S}_{i}\right)
$$

is called the Menger curve.

Example. There exists a convex-cocompact subgroup $G \subset \operatorname{Mob}\left(\mathbb{S}^{2}\right)$ whose limit set is homeomorphic to the Sierpinski carpet $\mathcal{S}$.

To construct such an example start with a compact hyperbolic manifold $M^{3}$ with nonempty totally-geodesic boundary. Thus we get an embedding of $\Gamma=$ $\pi_{1}\left(M^{3}\right)$ into $\mathbf{M o b}\left(\mathbb{S}^{2}\right)$ as a convex-cocompact Kleinian subgroup. The limit set of $\Gamma$ is homeomorphic to the Sierpinski carpet. To see this note that the convex hull $\operatorname{Hull}(\Lambda(\Gamma))$ in $\mathbb{H}^{3}$ is obtained by removing from $\mathbb{H}^{3}$ a countable collection of disjoint open half-spaces $H_{j} \subset \mathbb{H}^{3}$. The ideal boundary of each $H_{j}$ is the open round disk $D_{j} \subset \mathbb{S}^{2}$. Thus

$$
\Lambda(\Gamma)=\mathbb{S}^{2} \backslash \bigcup_{j} \operatorname{int}\left(D_{j}\right)
$$

Clearly, $D_{j} \cap D_{i}=\emptyset$, unless $i=j$; since $\Lambda(\Gamma)$ has empty interior. See Figure 5 . According to Claytor's theorem [57], it follows that $\Lambda(\Gamma)$ is homeomorphic to $\mathcal{S}$. Moreover, it is easy to see that this homeomorphism extends to the 2 -sphere, since it sends the boundary circles of $\Lambda(\Gamma)$ to the boundary squares of $\mathcal{S}$.

The construction of Kleinian groups whose limit sets are homeomorphic to $\mathcal{M}$ is more complicated:

Example. (M. Bourdon [37]; see also [118].) There exists a convex-cocompact subgroup $\Gamma \subset \operatorname{Mob}\left(\mathbb{S}^{3}\right)$ whose limit set is homeomorphic to the Menger curve $\mathcal{M}$.

The following theorem is proved in [122] in the more general context of Gromov-hyperbolic groups:

Theorem 8.1. (M. Kapovich, B. Kleiner [122].) Suppose that $\Gamma \subset \mathbf{M o b}\left(\mathbb{S}^{\mathrm{n}}\right)$ is a (torsion-free) nonelementary convex-cocompact subgroup such that:

(a) $\Gamma$ does not split as a free product,

(b) $\Gamma$ does not split as an amalgam over $\mathbb{Z}$,

(c) $\operatorname{dim}(\Lambda(\Gamma))=1$.

Then $\Lambda(\Gamma)$ is either homeomorphic to the Sierpinski carpet or to the Menger curve. 


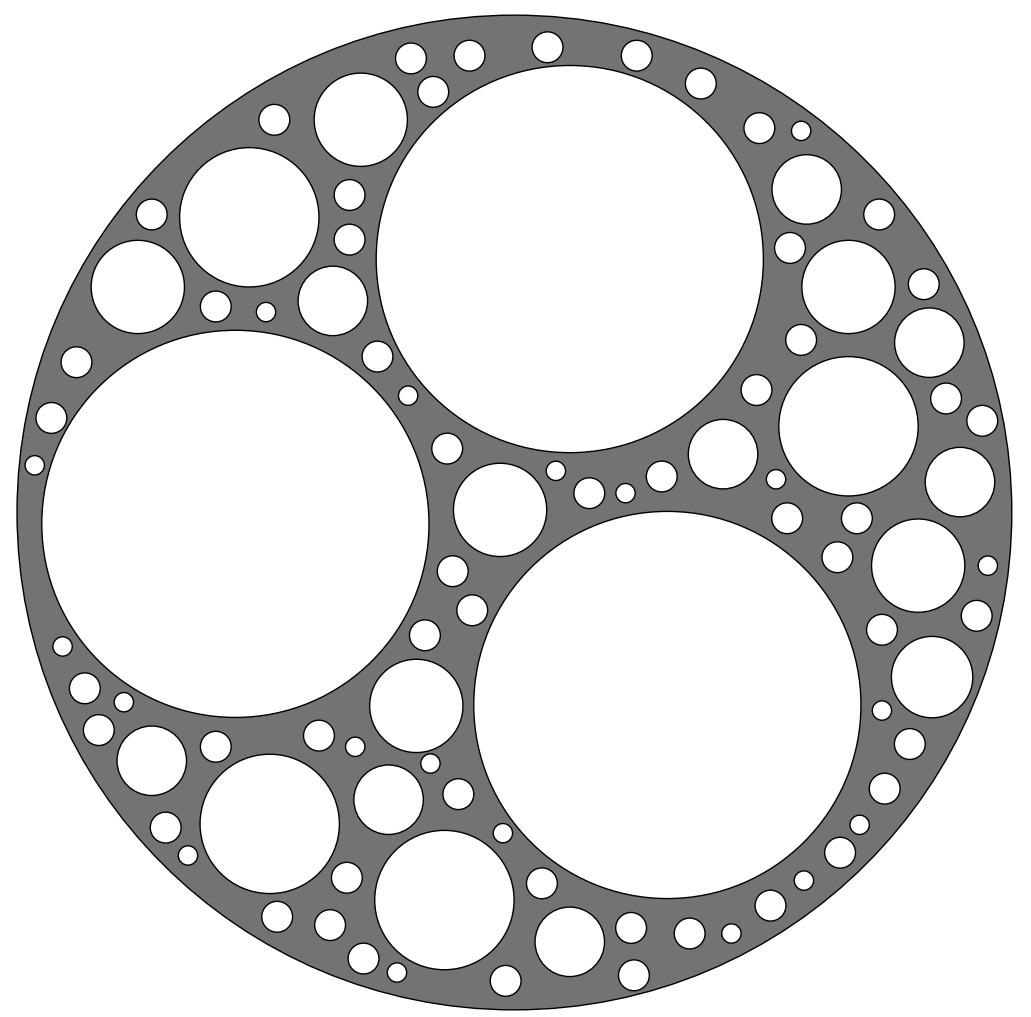

Figure 5. A limit set homeomorphic to the Sierpinski carpet.

Conjecture 8.2. (M. Kapovich, B. Kleiner [122].) If $\Gamma \subset \operatorname{Mob}\left(\mathbb{S}^{\mathrm{n}}\right)$ is a (torsionfree) convex-cocompact Kleinian group whose limit set is homeomorphic to the Sierpinski carpet, then $\Gamma$ is isomorphic to a convex-cocompact subgroup in $\operatorname{Mob}\left(\mathbb{S}^{2}\right)$.

It was proved in [122] that this conjecture would follow either from the positive solution of the 3-dimensional Wall's problem (Problem 4.5) or from Cannon's conjecture (Conjecture 4.6).

Topology of the limit sets of geometrically infinite Kleinian groups can be more complicated. A dendroid is a compact locally connected simply-connected 1-dimensional topological space.

Theorem 8.3. (J. Cannon and W. Thurston [51], see also [1] and [58].) There exist singly-degenerate Kleinian groups whose limit sets are dendroids.

Conjecturally, limit sets of all singly-degenerate Kleinian groups are dendroids and the following problem is open even for $n=2$ : 
Problem 8.4. Suppose that $\Gamma \subset \operatorname{Mob}\left(\mathbb{S}^{\mathrm{n}}\right)$ is a Kleinian group whose limit set is connected and 1-dimensional. Is it true that $\Lambda(\Gamma)$ is locally connected?

See [163], [165] and [168, 169] for partial results in dimension 2.

\section{Groups whose limit sets are topological spheres}

Definition 9.1. A Kleinian group $\Gamma \subset \operatorname{Mob}\left(\mathbb{S}^{n}\right)$ is called $i$-fuchsian ${ }^{2}$ if $\Lambda(\Gamma)$ is a round $i$-dimensional sphere in $\mathbb{S}^{n}$.

To construct examples of $i$-fuchsian groups start with a lattice $\Gamma \subset \operatorname{Mob}\left(\mathbb{S}^{i}\right)$. The limit set of $\Gamma$ is the round sphere $\mathbb{S}^{i}$. Define the canonical embedding

$$
\iota: \operatorname{Mob}\left(\mathbb{S}^{i}\right) \hookrightarrow \operatorname{Mob}\left(\mathbb{S}^{n}\right)
$$

induced by the embedding of the Lorentz groups

$$
\begin{gathered}
O(i+1,1) \hookrightarrow O(n+1,1) \\
A \mapsto\left[\begin{array}{cc}
A & 0 \\
0 & I
\end{array}\right],
\end{gathered}
$$

where $I$ is the identity matrix. Therefore we get the canonical embedding

$$
\iota: \Gamma \hookrightarrow \operatorname{Mob}\left(\mathbb{S}^{\mathrm{n}}\right) .
$$

One can modify this construction as follows. Note that the stabilizer of $\mathbb{S}^{i}$ in $\operatorname{Mob}\left(\mathbb{S}^{\mathrm{n}}\right)$ contains $\operatorname{Mob}\left(\mathbb{S}^{i}\right) \times S O(n-i)$. Choose a homomorphism $\phi: \Gamma \rightarrow S O(n-$ $i)$. For instance, being residually finite, the group $\Gamma$ will have many epimorphisms to finite groups, which then can be embedded in $S O(n-i)$ if $n-i$ is sufficiently large. Alternatively, in many cases the group $\Gamma$ will have infinite abelianization $\Gamma^{a b}$. The abelian group $\Gamma^{a b}$ admits many embeddings into $S O(n-i)$ provided that $n-i \geq 2$. Then the image of

$$
\rho=\iota \times \phi: \Gamma \rightarrow \operatorname{Mob}\left(\mathbb{S}^{i}\right) \times S O(n-i) \subset \operatorname{Mob}\left(\mathbb{S}^{\mathrm{n}}\right)
$$

is also an $i$-fuchsian group, since $\rho(\Gamma)$ preserves $\mathbb{S}^{i}$ and the action of $\rho(\Gamma)$ on $\mathbb{S}^{i}$ is the same as the action of $\Gamma$.

Definition 9.2. A Kleinian group $\Gamma \subset \operatorname{Mob}\left(\mathbb{S}^{n}\right)$ is called $i$-quasifuchsian if its limit set is a topological $i$-dimensional sphere.

We will refer to the number $n-i$ as the codimension of a (quasi)fuchsian group $\Gamma$.

Example. Suppose that $\Gamma$ is an $i$-fuchsian subgroup of $\mathbf{M o b}\left(\mathbb{S}^{n}\right)$ and $\Gamma^{\prime} \subset \operatorname{Mob}\left(\mathbb{S}^{n}\right)$ is another group which is topologically conjugate to $\Gamma$ (with a homeomorphism $f$ defined on the entire $n$-sphere). Then $\Gamma^{\prime}$ is $i$-quasifuchsian. However, as we will see, there are $i$-quasifuchsian groups (for $n \geq 3$ ) which cannot be obtained in this fashion.

${ }^{2}$ Our definition is somewhat different from the classical: fuchsian subgroups of $\operatorname{PSL}(2, \mathbb{C})$ are usually required to preserves a round disk in $\mathbb{S}^{2}$. 
The (quasiconformal) homeomorphisms $f$ as in the previous example exist in abundance if $i=1, n=2$, due to the solvability of the Beltrami equation. If $i \geq 2$, the situation is very different and it is not so easy to construct nontrivial examples of $i$-quasifuchsian groups which are not fuchsian. Some of these examples will be discussed below.

The following result was proved by M. Bestvina and G. Mess [28] in the context of Gromov-hyperbolic groups:

Theorem 9.3. Each convex-cocompact $i$-quasifuchsian group is a Poincaré duality group (over $\mathbb{Z}$ ) of dimension $i+1$. Conversely, if $\Gamma \subset \operatorname{Mob}\left(\mathbb{S}^{\mathrm{n}}\right)$ is a convexcocompact Poincaré duality group, then $\Lambda(\Gamma)$ is a homology manifold which is a homology sphere.

Question 9.4. Is it true that each convex-cocompact quasifuchsian group is isomorphic to the fundamental group of a closed aspherical manifold?

This is, of course, a special case of Wall's problem (Problem 4.5).

Question 9.5. Is there a convex-cocompact group $\Gamma \subset \operatorname{Mob}\left(\mathbb{S}^{\mathrm{n}}\right)$ whose limit set is a homology manifold which is homology sphere, so that $\Lambda(\Gamma)$ is not homeomorphic to a sphere?

\subsection{Quasifuchsian groups of codimension 1}

The situation in the case of $n=2$ is completely understood due to the following:

Theorem 9.6. (B. Maskit [156], see also [152].) Let $\Gamma \subset \mathbf{M o b}\left(\mathbb{S}^{2}\right)$ be a Kleinian group whose domain of discontinuity $\Omega(\Gamma)$ consists of precisely two components. Then:

1. $\Gamma$ is 1-quasifuchsian and geometrically finite.

2. $\Gamma$ is quasiconformally conjugate to a 1-fuchsian group.

3. $\bar{M}^{3}(\Gamma)=\left(\mathbb{H}^{3} \cup \Omega(\Gamma)\right) / \Gamma$ is homeomorphic to an interval bundle over a surface $S$, which is 2-fold covered by $\Omega(\Gamma) / \Gamma$.

Our goal is to compare the higher-dimensional situation with this theorem. Suppose that $\Gamma \subset \operatorname{Mob}\left(\mathbb{S}^{n}\right)$ is a codimension 1 quasifuchsian group. Then $\Omega(\Gamma)$ consists of two components, $\Omega_{1}, \Omega_{2}$. After replacing $\Gamma$ by an appropriate index 2 subgroup, we can assume that each $\Omega_{i}$ is $\Gamma$-invariant; hence $M^{n}(\Gamma)=M_{1} \cup M_{2}$, where $M_{i}:=\Omega_{i} / \Gamma$. Then, by the Alexander duality, $H_{*}\left(\Omega_{i}\right) \cong H_{*}($ point $), i=1,2$. Therefore, if $\Omega_{i}$ is simply-connected, then $\Omega_{i}$ is contractible. Below we discuss what is currently known about such quasifuchsian groups for $n \neq 4$.

Theorem 9.7. Suppose that both $M_{i}$ are compact and both $\Omega_{i}$ are simply-connected. Then $\bar{M}^{n+1}(\Gamma)$ is diffeomorphic to $M_{1} \times[0,1]$ provided that $n \geq 5$.

Proof. Note that, for homological reasons, $W=\bar{M}^{n+1}(\Gamma)$ is compact, hence $\Gamma$ is convex-cocompact, see Theorem 2.7. Since both $\Omega_{1}, \Omega_{2}, \mathbb{H}^{n+1}$ are contractible, the inclusions

$$
M_{i} \hookrightarrow W, \quad i=1,2
$$


are homotopy-equivalences. Therefore $W$ defines a smooth h-cobordism between the aspherical manifolds $M_{1}$ and $M_{2}$. According to Corollary 5.6, this h-cobordism is smoothly trivial.

Suppose that $n=3$ and both $\Omega_{1}, \Omega_{2}$ are contractible. Then

$$
\pi_{1}\left(M_{1}\right) \cong \pi_{1}\left(M_{2}\right) \cong \Gamma,
$$

the manifolds $M_{1}$ and $M_{2}$ are both irreducible and have infinite fundamental groups. If $\Gamma$ were to contain a subgroup $\Pi$ isomorphic to $\mathbb{Z}^{2}$, the subgroup $\Pi$ would be parabolic. This would contradict compactness of $W$. Therefore, according to Perelman's solution of Thurston's hyperbolization conjecture, there exists a closed hyperbolic 3-manifold $M_{0}$ which is homeomorphic to $M_{1}$ and $M_{2}$. Since $M_{0}$ is hyperbolic, its fundamental group $\Gamma_{0}$ acts as a 2 -fuchsian group on $\mathbb{S}^{3}$. Therefore, according to our discussion in Section 6 , the group $\Gamma$ is quasiconformally conjugate to $\Gamma_{0}$. It is not difficult to see that passage to the index 2 subgroup which we used above does no harm and we obtain:

Proposition 9.8. Suppose that $\Gamma \subset \mathbf{M o b}\left(\mathbb{S}^{3}\right)$ is a codimension 1 quasifuchsian subgroup, so that both components of $\Omega(\Gamma)$ are simply-connected and $M^{3}(\Gamma)$ is compact. Then $\Gamma$ is quasiconformally conjugate to a 2 -fuchsian group $\Gamma_{0} \subset \operatorname{Mob}\left(\mathbb{S}^{3}\right)$.

On the other hand, we do not know if the 4-dimensional manifold $\bar{M}(\Gamma)$ is homeomorphic (or diffeomorphic) to an interval bundle over a 3-manifold.

Proposition 9.8 fails for $n \geq 4$ :

Theorem 9.9. For every $n \geq 4$ there are codimension 1 convex-cocompact quasifuchsian subgroups $\Gamma \subset \operatorname{Mob}\left(\mathbb{S}^{n}\right)$, so that both components of $\Omega(\Gamma)$ are simplyconnected, but $\Gamma$ is not isomorphic to a fuchsian group.

Proof. We give only a sketch of the proof, the details will appear elsewhere. Fix $n \geq 4$. M. Gromov and W. Thurston in [89] construct examples of negatively curved compact conformally-flat $n$-manifolds $M^{n}$, so that $M^{n}$ is not homotopyequivalent to any closed hyperbolic $n$-manifold $N^{n}$. (See also [119] for a review of the Gromov-Thurston examples and for a construction of a convex projective structure on $M^{n}$.)

By choosing parameters in the construction of [89] more carefully, one can construct an example of a uniformizable flat conformal manifold $M^{n}$ with the same properties. Moreover, $M^{n}=\Omega_{1} / \Gamma, \Gamma \subset \operatorname{Mob}\left(\mathbb{S}^{n}\right)$ is convex-cocompact, and $\Omega(\Gamma)=\Omega_{1} \cup \Omega_{2}$ is the union of two simply-connected components. Then $\Lambda(\Gamma)$ is homeomorphic to $\mathbb{S}^{n-1}$, since the limit set of $\Gamma$ is homeomorphic to the ideal boundary of the universal cover of the negatively curved manifold $M^{n}$. If $\Gamma$ were isomorphic to an $n$-1-fuchsian group $\Gamma^{\prime}$, then $\Gamma^{\prime}$ would be isomorphic to the fundamental group of a closed hyperbolic $n$-manifold $N^{n}$, which is a contradiction.

The above examples have another interesting property. Let $\Omega^{n+1}$ denote the domain of discontinuity of the group $\Gamma$ (regarded as a subgroup of $\operatorname{Mob}\left(\mathbb{S}^{n+1}\right)$ ). 
Note that $\Omega^{n+1}$ is connected and $\pi_{1}\left(\Omega^{n+1}\right) \cong \mathbb{Z}$. Since both $\Omega_{1}, \Omega_{2}$ are contractible, it follows that

$$
\pi_{i}\left(\Omega^{n+1}\right)=0, \quad i \geq 2 .
$$

Set $M^{n+1}=\Omega^{n+1} / \Gamma$. We have the short exact sequence

$$
1 \rightarrow \mathbb{Z}=\pi_{1}\left(\Omega^{n+1}\right) \rightarrow \pi_{1}\left(M^{n+1}\right) \rightarrow \Gamma \rightarrow 1 .
$$

The embedding $M_{1} \rightarrow M^{n+1}$ determines a splitting of this sequence. Hence the manifolds $M^{n+1}$ and $\mathbb{S}^{1} \times M^{n}$ are homotopy-equivalent. Given the existence of a metric of negative curvature on $M^{n}$, we obtain a metric of nonpositive curvature on $\mathbb{S}^{1} \times M^{n}$. Therefore, by Theorem 5.4, the manifolds $M^{n+1}$ and $\mathbb{S}^{1} \times M^{n}$ are homeomorphic.

Let $k \mathbb{Z} \subset \mathbb{Z} \subset \pi_{1}\left(M^{n+1}\right)$ be the index $k$ subgroup in the center of $\pi_{1}\left(M^{n+1}\right)$. Then we obtain the $k$-fold covering $X_{k} \rightarrow X_{1}=M^{n+1}$, where

$$
\pi_{1}\left(X_{k}\right)=k \mathbb{Z} \times \Gamma \subset \pi_{1}\left(X_{1}\right) .
$$

Since the manifolds $X_{k}$ have isomorphic fundamental groups and $\pi_{i}\left(X_{k}\right)=0$ for all $i \geq 2, k \in \mathbb{N}$, these manifolds are all homeomorphic to the smooth manifold $X_{1}$ by Theorem 5.4. By [132, Essay IV], there only finitely many smooth structures on the manifold $X_{1}$. Therefore we obtain an infinite family of diffeomorphic manifolds

$$
M_{j}^{n+1}:=X_{k_{j}}, j \in \mathbb{N}
$$

and smooth covering maps $p_{j}: M_{j}^{n+1} \rightarrow M^{n+1}$.

The $(n+1)$-manifold $M^{n+1}=\Omega^{n+1} / \Gamma$ has the flat conformal structure $K_{1}$ uniformized by the group $\Gamma$. Let $K_{j}$ denote the flat conformal structure on $M^{n+1}$, which is the lift of $K_{1}$ via $p_{j} .{ }^{3}$ We thus obtain an infinite family of diffeomorphic flat conformal manifolds

$$
\left(M_{j}^{n+1}, K_{j}\right), j=1,2, \ldots
$$

Question 9.10. Suppose that $M$ is a closed hyperbolic n-manifold. Is there a finite cover $f: M^{\prime} \rightarrow M$ such that the pull-back map $f^{*}: H^{3}(M, \mathbb{Z} / 2) \rightarrow H^{3}\left(M^{\prime}, \mathbb{Z} / 2\right)$ is trivial? (Recall $[132]$ that the group $H^{3}(M, \mathbb{Z} / 2)$ classifies $P L$ structures on $M$ if $n \geq 5$.)

We regard the structures $K_{j}$ as elements of $\mathfrak{M}(X)$, the moduli space of the flat conformal structures on a fixed smooth manifold $X$. The proof of the following claim is similar to [108], where it was proved in the context of 3-manifolds:

Proposition 9.11. For different $i, j$ the structures $K_{i}, K_{j}$ lie in different connected components of the moduli space $\mathfrak{M}(X)$. Thus $\mathfrak{M}(X)$ consists of infinitely many connected components.

We note that K. Scannell in [197] constructed examples of hyperbolic 3manifolds $X$ for which $\mathfrak{M}(X)$ consists of infinitely many connected components.

\footnotetext{
${ }^{3}$ The structures $K_{j}$ are obtained via grafting of $\left(M^{n+1}, K_{1}\right)$ along the hypersurface $M^{n}$.
} 
To get the same phenomenon in dimension 4 consider one of the hyperbolic manifolds $M^{3}$ obtained by Dehn surgery on a 2-bridge knot, so that the natural embedding

$$
\Gamma=\pi_{1}(M) \hookrightarrow \operatorname{Mob}\left(\mathbb{S}^{3}\right)
$$

is (locally) rigid (see Example 11.1.2). Then the natural embedding of $\Gamma \rightarrow$ $\operatorname{Mob}\left(\mathbb{S}^{4}\right)$ is also rigid and hence the manifold $M^{4}=M^{4}(\Gamma) \cong M \times \mathbb{S}^{1}$ has rigid flat conformal structure. Taking $k$-fold covers of this manifold we obtain infinitely many rigid flat conformal structures on $M^{4}$. By combining these results we obtain

Theorem 9.12. For every $n \geq 3$ there exists a smooth compact $n$-manifold $X^{n}$ such that $\mathfrak{M}\left(X^{n}\right)$ consists of infinitely many connected components.

We now return to our discussion of Kleinian groups, restricting to $n=3$. Suppose that $\Gamma$ is a convex-cocompact 2-quasifuchsian group, such that both components of $\Omega(\Gamma)$ are simply-connected. Then, by proposition 9.8 , the limit set of $\Gamma$ is tame, i.e., there is a homeomorphism of $\mathbb{S}^{3}$ which maps $\Lambda(\Gamma)$ to the round sphere.

Theorem 9.13. (B.Apanasov and A. Tetenov [11].) There exists a convex-cocompact 2-quasifuchsian group $\Gamma \subset \operatorname{Mob}\left(\mathbb{S}^{3}\right)$ whose limit set is a wild 2-sphere, i.e., there is no homeomorphism of $\mathbb{S}^{3}$ which maps $\Lambda(\Gamma)$ to the round sphere. Moreover, one component of $\Omega(\Gamma)$ is simply-connected.

\subsection{1-quasifuchsian subgroups of $\operatorname{Mob}\left(\mathbb{S}^{3}\right)$}

Given a Kleinian subgroup $\Gamma \subset \operatorname{Mob}\left(\mathbb{S}^{3}\right)$ whose limit set is a topological circle $C$, we would like to analyze the embedding $C \hookrightarrow \mathbb{S}^{3}$. It is clear that $C$ could be an unknot in $\mathbb{S}^{3}$ (i.e., there exists a homeomorphism of $\mathbb{S}^{3}$ which maps $C$ to a round circle), take for instance any 1-fuchsian subgroup of $\mathbf{M o b}\left(\mathbb{S}^{3}\right)$.

A topological circle $C$ in $\mathbb{S}^{3}$ is called tame if it is isotopic to a polygonal knot in $\mathbb{S}^{3}$; if $C$ is not tame, it is called wild.

Proposition 9.14. 1. If $\Gamma$ is a 1-quasifuchsian subgroup of $\operatorname{Mob}\left(\mathbb{S}^{3}\right)$, then either $\Lambda(\Gamma)$ is an unknot or it is a wild knot $K$ such that $\pi_{1}\left(\mathbb{S}^{3} \backslash K\right)$ is infinitely generated.

2. Each 1-quasifuchsian group is geometrically finite.

Proof. Since $\Gamma$ is a 1-quasifuchsian subgroup of $\operatorname{Mob}\left(\mathbb{S}^{3}\right)$, this group is nonelementary. The fundamental group of $M=M^{3}(\Gamma)$ is finitely generated (since $\Gamma$ is) and we have the exact sequence:

$$
1 \rightarrow \pi_{1}(\Omega(\Gamma)) \rightarrow \pi_{1}(M) \rightarrow \Gamma \rightarrow 1 .
$$

Suppose that $\pi_{1}(\Omega(\Gamma))$ is finitely generated. Then, according to Jaco-Hempel's Theorem $[95], \pi_{1}(\Omega(\Gamma)) \cong \mathbb{Z}$. This immediately excludes tame nontrivial knots (the result proved by R. Kulkarni [140]). It remains to exclude wild knots with

$$
\Delta:=\pi_{1}(\Omega(\Gamma)) \cong \mathbb{Z} .
$$


Without loss of generality (after passing to an index 2 subgroup in $\Gamma$ ), we can assume that $\Delta$ is contained in the center of $\pi_{1}(M)$. Note that $M$ is a Seifert manifold since its fundamental group has infinite center. Hence $M$ admits an $\mathbb{S}^{1}$-action. Lift this action to $\Omega(\Gamma)$ and then extend it to the entire 3-sphere (so that the fixed point set is the limit set). Raymond's classification [192] of topological $\mathbb{S}^{1}$-actions on $\mathbb{S}^{3}$ implies that this $\mathbb{S}^{1}$-action is topologically conjugate to the orthogonal action, hence $\Lambda(\Gamma)$ is an unknot. This proves (1).

To prove (2) note that the group $\Gamma$ acts as a convergence group on $\mathbb{S}^{1}=\Lambda(\Gamma)$ (see [218]). Hence, according to [218], there exists a homeomorphism $f: \Lambda(\Gamma) \rightarrow \mathbb{S}^{1}$ such that

$$
f \Gamma f^{-1}=\Gamma^{\prime} \subset P S L(2, \mathbb{R}) .
$$

Since finitely generated discrete subgroups of $\operatorname{PSL}(2, \mathbb{R})$ are geometrically finite, it follows that $\Gamma^{\prime}$ is geometrically finite. As geometric finiteness is an invariant of the topological dynamics on the limit set (see Theorem 2.5), the group $\Gamma$ is geometrically finite as well.

On the other hand, even if $\Lambda(\Gamma)$ is an unknot, the 3 -manifold $\Omega(\Gamma) / \Gamma$ is not necessarily a product:

Theorem 9.15. (M. Gromov, B. Lawson, W. Thurston [87], N. Kuiper [139], and M. Kapovich $[107,112]$.) There are 1-quasifuchsian groups $\Gamma \subset \mathbf{M o b}\left(\mathbb{S}^{3}\right)$ such that $\Lambda(\Gamma)$ are unknotted but $\Gamma$ are not topologically conjugate to 1-fuchsian groups.

In the examples constructed in this theorem, the manifolds $M^{3}(\Gamma)$ are nontrivial oriented circle bundles over orientable surfaces. On the other hand, for every 1-fuchsian group $\Gamma_{0} \subset \operatorname{Mob}\left(\mathbb{S}^{3}\right)$, the manifold $M^{3}\left(\Gamma_{0}\right)$ is a 3-dimensional Seifert manifold with the zero Euler number, since it admits a natural $\mathbb{H}^{2} \times \mathbb{R}$-structure. Hence, in this case, $M^{3}\left(\Gamma_{0}\right)$ admits a finite cover which is homeomorphic to the product of $\mathbb{S}^{1}$ and a surface.

Theorem 9.16. (B. Apanasov [10], B. Maskit [157], see also [87].) There are 1quasifuchsian groups $\Gamma \subset \operatorname{Mob}\left(\mathbb{S}^{3}\right)$ such that $\Lambda(\Gamma)$ are wild knots.

Proof. (Sketch.) Start with a necklace of round balls

$$
B_{0}, B_{1}, \ldots, B_{m-1} \subset \mathbb{S}^{3},
$$

so that $B_{i}$ is tangent to $B_{j}$, if $j=i+1 \in \mathbb{Z} / m \mathbb{Z}$ and is disjoint otherwise. Assume that this necklace is knotted, i.e., the polygonal knot obtained by connecting the consecutive points of tangency is a nontrivial knot $K \subset \mathbb{S}^{3}$. See Figure 6 .

Let $\gamma_{i} \in \operatorname{Mob}\left(\mathbb{S}^{3}\right)$ denote the inversion in the sphere $\partial B_{i}, i=0,1, \ldots, m-1$. Let $\Gamma \subset \operatorname{Mob}\left(\mathbb{S}^{n}\right)$ be the group generated by these inversions. By the Poincaré fundamental polyhedron theorem,

$$
\Phi=\mathbb{S}^{3} \backslash \bigcup_{i=0}^{m-1} B_{i}
$$




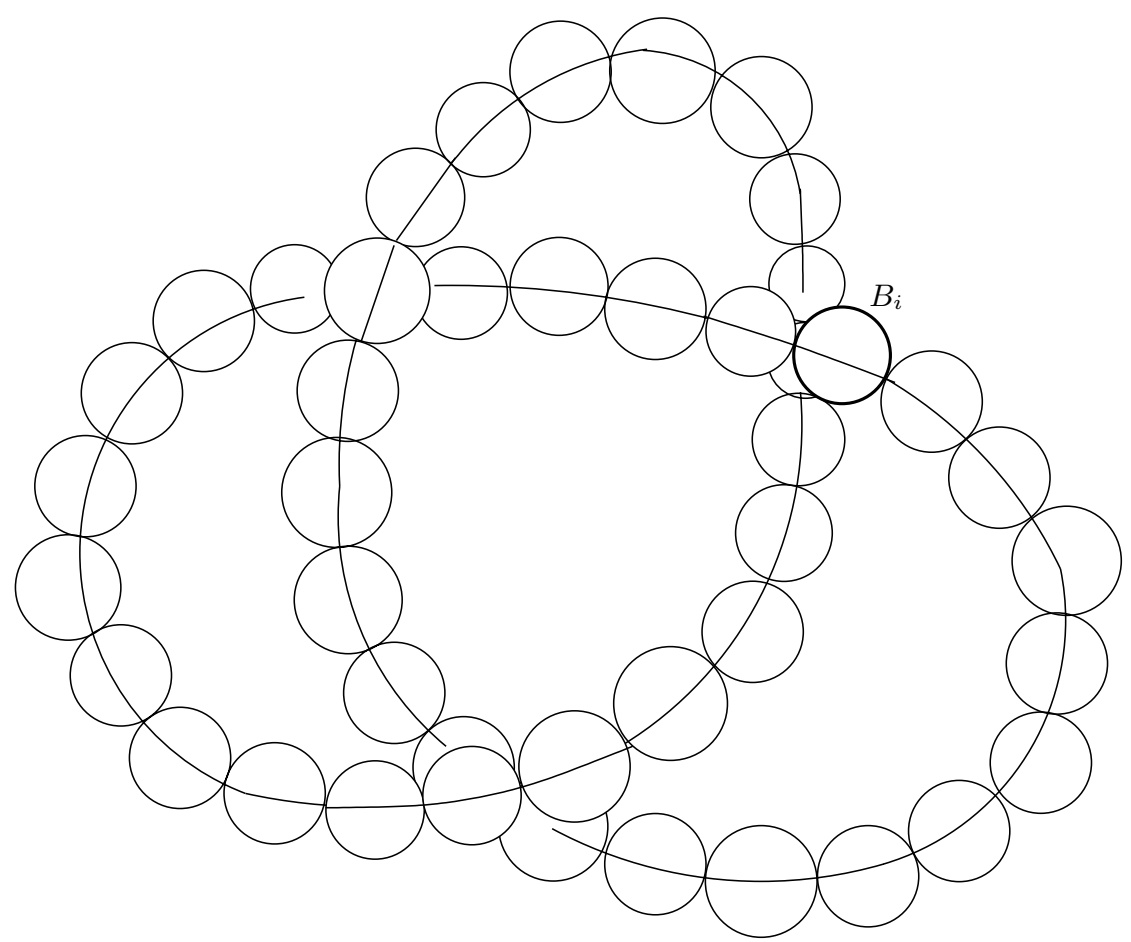

FigURE 6

is a fundamental domain for $\Gamma$, the group $\Gamma$ is geometrically finite and is isomorphic to a 1-fuchsian group $\Gamma^{\prime}$. Therefore, Tukia's theorem 6.1 applied to the isomorphism $\Gamma \rightarrow \Gamma^{\prime}$, implies that $\Gamma$ is 1-quasifuchsian. By Seifert-van Kampen Theorem, $\pi_{1}\left(\mathbb{S}^{3} \backslash K\right)$ embeds in $\pi_{1}(\Omega(\Gamma))$. Therefore $\pi_{1}(\Omega(\Gamma))$ is not isomorphic to $\mathbb{Z}$ and, hence, the limit set of $\Gamma$ is a wild knot.

By modifying the above construction, S. Hwang proved

Theorem 9.17. (S. Hwang [97].) Let $L$ be a polygonal link in $\mathbb{S}^{3}$. Then there exists a (torsion-free) convex-cocompact Kleinian group $\Gamma \subset \operatorname{Mob}\left(\mathbb{S}^{3}\right)$ with a fundamental domain $\Phi \subset \mathbb{S}^{3}$ such that the complement $\mathbb{S}^{3} \backslash \Phi$ is isotopic to a regular neighborhood of $L$.

The above theorem is the key for proving

Theorem 9.18. (S. Hwang [97].) Let $M^{3}$ be a closed oriented 3-manifold. Then there exists a closed oriented 3 -manifold $N^{3}$ such that the connected sum $M^{3} \# N^{3}$ admits a Möbius structure. 
Results similar to Theorems 9.17 and 9.18 hold in dimension 4, see [117], although one has to assume that $M^{4}$ is a Spin-manifold. One of the key ingredients in [117] is the following:

Theorem 9.19. Let $Q \subset \mathbb{R}^{4} \subset \mathbb{S}^{4}$ be a finite 2-dimensional subcomplex in the standard cubulation of $\mathbb{R}^{4}$. Then there exists a convex-cocompact Kleinian subgroup $\Gamma \subset \operatorname{Mob}\left(\mathbb{S}^{4}\right)$ (generated by reflections) with a fundamental domain $\Phi \subset \mathbb{S}^{4}$, such that the complement $\mathbb{S}^{4} \backslash \Phi$ is isotopic to a regular neighborhood of $Q$.

Very little is known about quasifuchsian groups in $\mathbf{M o b}\left(\mathbb{S}^{n}\right)$ whose limit sets have dimension between 2 and $n-2$. Perhaps the most interesting result here is obtained by I. Belegradek [15] who used the construction from [87] to get

Theorem 9.20. There exist convex-cocompact 2-quasifuchsian subgroups $\Gamma_{1}, \Gamma_{2} \subset$ $\operatorname{Mob}\left(\mathbb{S}^{4}\right)$ so that:

1) $\Lambda\left(\Gamma_{1}\right)$ is a wild 2-sphere in $\mathbb{S}^{4}$.

2) $\Lambda\left(\Gamma_{2}\right)$ is tame but the group $\Gamma_{2}$ is not topologically conjugate to a 2-fuchsian group: $M^{4}\left(\Gamma_{2}\right)$ is a nontrivial circle bundle over a hyperbolic 3-manifold.

Similar results probably hold for codimension 2 quasifuchsian subgroups in $\operatorname{Mob}\left(\mathbb{S}^{\mathrm{n}}\right), n \geq 5$.

\section{Ahlfors finiteness theorem in higher dimensions: Quest for the holy grail}

\subsection{The holy grail}

One of the most fundamental results of the theory of Kleinian subgroups of $\operatorname{Mob}\left(\mathbb{S}^{2}\right)$ is the Ahlfors Finiteness Theorem (the "Holy Grail"), which we state here together with its companions:

Theorem 10.1. Suppose that $\Gamma \subset P S L(2, \mathbb{C})$ is a Kleinian group ${ }^{4}$ which may have torsion. Then the following hold:

1. (L. Ahlfors [3], L. Greenberg [83].) The group $\Gamma$ is analytically finite, i.e., the quotient $O:=\Omega(\Gamma) / \Gamma$ is a complex orbifold of finite conformal type ${ }^{5}$. In particular, $O$ is homotopy-equivalent to a finite $C W$ complex.

2. (D. Sullivan [209].) $\Gamma$ has only finitely many cusps.

3. (M. Feighn and G. Mess [75].) $\Gamma$ has only finitely many $\Gamma$-conjugacy classes of finite order elements.

4. (P.Scott $[201,202].) \Gamma$ is finitely presentable and the orbifold $\mathbb{H}^{3} / \Gamma$ is finitely covered by a manifold $\mathbb{H}^{3} / \Gamma^{\prime}$, which is homotopy-equivalent to a compact 3 manifold.

${ }^{4}$ Recall that all Kleinian groups are assumed to be finitely generated.

${ }^{5}$ I.e., as a Riemann surface it biholomorphic to a compact Riemann surface with a finite subset removed; as an orbifold it has only finitely many singular cone-points. 
5. (L. Ahlfors [4].) The action of $\Gamma$ on $\Lambda(\Gamma)$ is recurrent with respect to the Lebesgue measure $\mu$.

Alternative analytical proofs of Part 1 (i.e., the original Ahlfors' finiteness theorem) are given for instance in [116, Section 8.14] and [153]. A geometric proof (valid even in the context of manifolds of negative curvature) follows from the solution of Tameness Conjecture, see [2]

Corollary 10.2. If $\Gamma$ is as above, then:

(a) For each component $\Omega_{0}$ of $\Omega(\Gamma)$, the limit set of the stabilizer of $\Omega_{0}$ in $\Gamma$ equals $\partial \Omega_{0}$ (follows directly from Part 1 of Theorem 10.1). In particular, no component of $\Omega(\Gamma)$ has trivial stabilizer.

(b) Kleinian subgroups $\Gamma$ of $\operatorname{Mob}\left(\mathbb{S}^{2}\right)$ are coherent, i.e., each finitely generated subgroup of $\Gamma$ is also finitely presented (follows from Part 4 of Theorem 10.1).

(c) (W. Thurston, see [170].) If $\Gamma \subset \operatorname{Mob}\left(\mathbb{S}^{2}\right)$ is geometrically finite with $\Omega(\Gamma) \neq$ $\emptyset$, then each finitely generated subgroup $\Delta \subset \Gamma$ is geometrically finite as well.

We also now fully understand the topology of the manifold (orbifold) $\mathbb{H}^{3} / \Gamma$ :

Theorem 10.3 (Former tameness conjecture). The quotient $\mathbb{H}^{3} / \Gamma$ is tame, i.e., it is homeomorphic to the interior of a compact manifold (orbifold) with boundary.

The above theorem was proved for freely indecomposable groups $\Gamma$ by $\mathrm{F}$. Bonahon [34] and by I. Agol [2], D. Calegari and D. Gabai [49] in the general case.

The next theorem is a combination of a result by Thurston [213], who proved ergodicity for tame Kleinian subgroups of $\operatorname{Mob}\left(\mathbb{S}^{2}\right)$, and the proof of the tameness conjecture:

Theorem 10.4. If $\Gamma$ is as above, then the action of $\Gamma$ on $\Lambda(\Gamma)$ is ergodic with respect to the Lebesgue measure: each measurable $\Gamma$-invariant function on $\Lambda(\Gamma)$ is constant a.e.

Note, that the conglomerate of assertions presented above contains statements of different nature: algebraic, topological, dynamical. For a while it was hoped that a theorem analogous to Theorem 10.1 can be proved for Kleinian groups in higher dimensions; an attempt to develop analytical technique to achieve this was made by Ahlfors in [6] (see also [179]).

Nearly all algebraic and topological assertions of Theorem 10.1 and the Corollary 10.2 have been disproved in the case of Kleinian groups acting in higher dimensions (M. Kapovich and L. Potyagailo [114], [127], [128], [187], [188]):

Theorem 10.5. There exist Kleinian subgroups $\Gamma_{1}, \ldots, \Gamma_{5} \subset \mathbf{M o b}\left(\mathbb{S}^{3}\right)$ so that:

1. The group $\Gamma_{1}$ is not finitely presentable.

2. For each $i$, the manifold $M\left(\Gamma_{i}\right)=\Omega\left(\Gamma_{i}\right) / \Gamma_{i}$ contains a component with infinitely generated fundamental group.

3. $\Gamma_{2}$ is free and has infinitely many cusps (of rank 1 ). 
4. $\Gamma_{3}$ is not torsion-free and has infinitely many conjugacy classes of finite order elements.

5. $\Gamma_{4}$ is a normal subgroup of a convex-cocompact group $\widehat{\Gamma}_{4} \subset \operatorname{Mob}\left(\mathbb{S}^{3}\right)$ and satisfies (1), (2) and (4).

6. (B. Bowditch, G. Mess [42].) The group $\Gamma_{5}$ satisfies (1) and (2) and is contained in a cocompact lattice $\widehat{\Gamma}_{5} \subset \operatorname{Mob}\left(\mathbb{S}^{3}\right)$.

7. Groups $\Gamma_{i}, i=1, \ldots, 4$ are normal subgroups of geometrically finite groups $\widehat{\Gamma}_{i}$ so that $\widehat{\Gamma}_{i} / \Gamma_{i} \cong \mathbb{Z}$.

Remark 10.6. By modifying $\Gamma_{3}$ one can also construct an example $\Gamma_{6} \subset \operatorname{Mob}\left(\mathbb{S}^{3}\right)$ such that $\Omega\left(\Gamma_{6}\right) / \Gamma_{6}$ has infinitely many connected components.

At the time when the above examples were constructed, they were regarded as a "rare pathology". It appears however that such examples are rather common:

Conjecture 10.7. (M. Kapovich, L. Potyagailo, E. Vinberg [129].) Suppose that $\Gamma \subset \operatorname{Mob}\left(\mathbb{S}^{\mathrm{n}}\right)$ is an arithmetic lattice, where $n \geq 3$. Then $\Gamma$ is noncoherent, i.e., it contains a finitely generated subgroup $\Delta$ which is not finitely presentable.

This conjecture was proved in [129] in a number of special cases, e.g., for all non-cocompact arithmetic lattices provided that $n \geq 5$.

All the examples $\Gamma_{i}$ in the above theorem are based upon the existence of hyperbolic 3-manifolds $M^{3}$ of finite volume which fiber over the circle: the groups $\Gamma_{i}$ are obtained by manipulating with the normal surface subgroups in $\pi_{1}\left(M^{3}\right)$.

Problem 10.8. Find examples similar to $\Gamma_{i}$ 's without using hyperbolic 3-manifolds fibering over the circle.

Problem 10.9. Construct a finitely generated Kleinian subgroup $\Gamma \subset \operatorname{Mob}\left(\mathbb{S}^{\mathrm{n}}\right)$ such that Part (a) of Corollary 10.2 fails for $\Gamma$.

Problem 10.10. (G. Mess.) Construct a finitely-presented Kleinian subgroup of $\operatorname{Mob}\left(\mathbb{S}^{n}\right)(n \geq 3)$ which contains no parabolic elements and for which any of the assertions of Theorem 10.1 fail. (In Part (a) one would need to replace analytical finiteness with finiteness of the homotopy type.)

Problem 10.11. Construct a finitely generated Kleinian subgroup $\Gamma \subset \operatorname{Mob}\left(\mathbb{S}^{3}\right)$ such that $\Omega(\Gamma)$ contains a contractible component $\Omega_{0}$ so that:

The stabilizer $\Gamma_{0}$ of $\Omega_{0}$ in $\Gamma$ is finitely-generated, but the manifold $\Omega_{0} / \Gamma_{0}$ is not tame.

Note however that although algebra and topology fail, the assertions of dynamical nature (part 5 of Theorem 10.1, part (a) of Corollary 10.2, and Theorem 10.4) remain open in higher dimensions. Moreover, an attempt to construct a higher-dimensional counter-example to Theorem 10.1 (part 5) along the lines of the examples $\Gamma_{i}$, is doomed to failure: 
Theorem 10.12. (K. Matsuzaki [160].) Let $\widehat{\Gamma}$ be a geometrically finite subgroup ${ }^{6}$ in $\operatorname{Mob}\left(\mathbb{S}^{\mathrm{n}}\right)$. Suppose that $\Gamma \subset \widehat{\Gamma}$ is a normal subgroup (which does not have to be finitely generated). Then the action of $\Gamma$ on its limit set is recurrent.

Ergodicity fails however for discrete subgroups of $P U(2,1)$ (it probably also fails for Kleinian groups in higher dimensions but an example would be difficult to construct):

Theorem 10.13. There exists a finitely generated (but not finitely presentable!) discrete group $\Gamma$ of isometries of complex-hyperbolic 2 -plane $\mathbb{C H}^{2}$ so that the limit set of $\Gamma$ is the 3-sphere and the action of $\Gamma$ on $\mathbb{S}^{3}$ is not ergodic.

Proof. There are examples (the first was constructed by R. Livne in his thesis [145], see also [62]) of cocompact torsion-free discrete subgroups $\widehat{\Gamma} \subset P U(2,1)$ such that the complex 2-manifold $M=\mathbb{C H}^{2} / \widehat{\Gamma}$ admits a nonconstant holomorphic map $f: M \rightarrow S$ to a Riemann surface $S$ of genus $\geq 2$. The fundamental group of the generic fiber of $f$ maps onto a normal subgroup $\Gamma$ in $\widehat{\Gamma}$, so that $\Gamma$ is finitely generated but is not finitely presentable [115]. By lifting $f$ to the universal covers we get a nonconstant holomorphic $\Gamma$-invariant function

$$
\tilde{f}: \mathbb{C H}^{2} \rightarrow \mathbb{H}^{2} \text {. }
$$

Then the bounded harmonic function $\operatorname{Re}(\tilde{f})$ is also $\Gamma$-invariant and nonconstant. This harmonic function admits a measurable extension $h$ to $\mathbb{S}^{3}$, the boundary of the complex ball $\mathbb{C H}^{2}$, so that $h$ is $\Gamma$-invariant and not a.e. constant.

\subsection{Groups with small limit sets}

So far, our quest for the holy grail mostly resembled Monty Python's: We are not sure what to look for in higher dimensions. Nevertheless, there is a glimmer of hope.

Recall that the Hausdorff dimension $\operatorname{dim}_{H}$ of a subset $E \subset \mathbb{R}^{n}$ is defined as follows. For each $\alpha>0$ consider the $\alpha$-Hausdorff measure of $E$ :

$\operatorname{mes}_{\alpha}(E)=\lim _{\rho \rightarrow 0} \inf \left\{\sum_{i} r_{i}^{\alpha}: \quad r_{i} \leq \rho, E\right.$ is contained in the union of $r_{i}$-balls $\}$.

The Hausdorff dimension of $E$ is

$$
\operatorname{dim}_{H}(E)=\inf _{\alpha}\left\{\alpha: \operatorname{mes}_{\alpha}(E)=0\right\} .
$$

According to [96], for every bounded subset $E \subset \mathbb{R}^{n}$ one has the inequality

$$
\operatorname{dim}(E) \leq \operatorname{dim}_{H}(E)
$$

between topological and Hausdorff dimensions. In particular, if $\Gamma$ is a Kleinian group with $\operatorname{dim}_{H}(\Lambda(\Gamma))<1$, then $\Gamma$ is geometrically finite and is isomorphic to a Schottky-type group, see Theorem 7.7.

Conjecture 10.14. If $\operatorname{dim}_{H}(\Lambda(\Gamma))<1$, then $\Gamma$ is a Schottky-type group. Moreover, $\Gamma$ is classical.

${ }^{6}$ Actually, the proof also works for subgroups of any rank 1 Lie group. 
The critical exponent of a Kleinian group $\Gamma \subset \operatorname{Mob}\left(\mathbb{S}^{n}\right)$ is

$$
\delta(\Gamma):=\inf \left\{s>0: \sum_{\gamma \in \Gamma} e^{-s d(x, \gamma x)}<\infty\right\},
$$

where $d$ is the hyperbolic metric in $\mathbb{H}^{n+1}$. The following theorem is the result of combined efforts of a large number of mathematicians, including P. Tukia, D. Sullivan and P. Nicholls, we refer to [177], [33] for the proofs:

Theorem 10.15. For every Kleinian subgroup $\Gamma \subset \operatorname{Mob}\left(\mathbb{S}^{\mathrm{n}}\right)$,

$$
\delta(\Gamma)=\operatorname{dim}_{H}\left(\Lambda_{c}(\Gamma)\right) .
$$

Recall that $\Lambda_{c}(\Gamma)$ is the conical limit set of $\Gamma$.

The critical exponent relates to $\lambda_{0}$, the bottom of the spectrum of Laplacian on the hyperbolic manifold $\mathbb{H}^{n+1} / \Gamma$, by the following

Theorem 10.16. (D. Sullivan [211])

$$
\begin{gathered}
\lambda_{0}=\left(\frac{n}{2}\right)^{2}, \text { if } \quad 0 \leq \delta(\Gamma) \leq \frac{n}{2}, \\
\lambda_{0}=\delta(\Gamma)(n-\delta(\Gamma)), \text { if } \quad \frac{n}{2}<\delta(\Gamma) \leq n .
\end{gathered}
$$

The expectation is that Kleinian groups in $\operatorname{Mob}\left(\mathbb{S}^{n}\right)$ with small limit sets behave analogously to the Kleinian subgroups of $\mathbf{M o b}\left(\mathbb{S}^{2}\right)$.

Conjecture 10.17. Suppose that $\Gamma$ is a (finitely generated) subgroup of $\mathbf{M o b}\left(\mathbb{S}^{\mathrm{n}}\right)$ so that $\Lambda(\Gamma)$ has Hausdorff dimension $<2$. Then $\Gamma$ is geometrically finite.

For $n=2$, this conjecture is a theorem of C. Bishop and P. Jones [33]. A partial generalization of [33] was proved by A. Chang, J. Qing, J. and P. Yang [54]:

Theorem 10.18. Suppose that $\Gamma$ is a (finitely generated) conformally finite ${ }^{7}$ subgroup of $\mathbf{M o b}\left(\mathbb{S}^{n}\right)$ such that $\operatorname{dim}_{H}(\Lambda(\Gamma))<n$. Then $\Gamma$ is geometrically finite.

The converse to the above theorem was proved earlier by P. Tukia [216].

Theorem 10.19. (Y.Shalom [204].) Suppose that $\Gamma$ is a geometrically finite subgroup of $\operatorname{Mob}\left(\mathbb{S}^{n}\right)$ such that $\operatorname{dim}_{H}(\Lambda(\Gamma))<2$ and $\Delta \subset \Gamma$ is a finitely generated normal subgroup. Then $\Delta$ has finite index in $\Gamma$. In particular, $\Delta$ is geometrically finite as well.

Thus, attempts to construct geometrically infinite groups using normal subgrooups in geometrically finite Kleinian groups with small limit sets, are doomed to failure. On the other hand, the assumption that $\delta(\Gamma)$ is small should impose strong restrictions on the algebraic properties of the group $\Gamma$.

Conjecture 10.20. Suppose that $\Gamma$ is a Kleinian group in $\operatorname{Mob}\left(\mathbb{S}^{\mathrm{n}}\right)$ which does not contain parabolic elements. Then:

${ }^{7}$ I.e., $M^{n}(\Gamma)=\Omega(\Gamma) / \Gamma$ is compact modulo cusps. 
1. $c d(\Gamma)-1 \leq \delta(\Gamma)$.

2. In the case of equality, $\Gamma$ is an $i$-fuchsian convex-cocompact group, $i=\delta(\Gamma)$.

Recall that $c d$ and $h d$ stand for the cohomological and homological dimensions of a group. A partial confirmation of Part 1 of this conjecture is obtained in

Theorem 10.21. (M. Kapovich [121].) Suppose that $\Gamma \subset \operatorname{Mob}\left(\mathbb{S}^{n}\right)$ is a Kleinian group. Then for every ring $R$,

$$
h d_{R}(\Gamma, \Pi)-1 \leq \delta(\Gamma),
$$

where $\Pi \subset \Gamma$ is the set of virtually abelian subgroups of $\Gamma$ of (virtual) rank $\geq 2$ and $h d_{R}(\Gamma, \Pi)$ is the relative homological dimension.

We refer the reader to the series of papers by H. Izeki $[100,101,102]$ for the related results.

Corollary 10.22. (M. Kapovich [121].) Suppose that the group $\Gamma$ is finitely-presented and $\delta(\Gamma)<1$. Then $\Gamma$ is free.

Proof. Since $\delta(\Gamma)<1$, it follows that $\Gamma$ contains no rank 2 abelian subgroups. Then we have the inequalities

$$
c d(\Gamma) \leq 1+h d(\Gamma) \leq \delta(\Gamma)+2<3 .
$$

Combined with finite presentability of $\Gamma$, the inequality $\operatorname{cd}(\Gamma) \leq 2$ implies that $\Gamma$ has finite type; therefore

$$
c d(\Gamma)=h d(\Gamma) \leq \delta(\Gamma)+1<2 .
$$

Hence $\Gamma$ is free by Stallings' Theorem 4.1.

An inequality similar to Conjecture 10.20 was proved by A. Reznikov: For a (finitely-generated) group $\Gamma$ define

$$
\alpha(\Gamma):=\inf \left\{p \in[1, \infty]: \ell_{p} H^{1}(\Gamma) \neq 0\right\} .
$$

Here $\ell_{p} H^{1}$ is the first $\ell_{p}$-cohomology of the group $\Gamma$, see [38] for the precise definition. Then

Theorem 10.23. (A. Reznikov [194], see also [38] for the detailed proof in the case of isometries of $C A T(-1)$ spaces.) For every Kleinian group $\Gamma \subset \operatorname{Mob}\left(\mathbb{S}^{n}\right)$,

$$
\alpha(\Gamma) \leq \max (\delta(\Gamma), 1) .
$$

Question 10.24. What can be said about $\Gamma$ in the case of equality in Reznikov's theorem?

In the case of geometrically finite groups, Part 2 of Conjecture 10.20 holds:

Theorem 10.25. $\quad$ 1. (Chenbo Yue [228], see also [22] and [35].) Suppose that $\Gamma$ is convex-cocompact and $i=\delta(\Gamma)=c d(\Gamma)-1$. Then $\Gamma$ is $i$-fuchsian. 
2. (M. Kapovich [121].) Suppose that $\Gamma$ is geometrically finite. Then the following three conditions are equivalent:

$$
\begin{aligned}
& i=\delta(\Gamma)=c d(\Gamma, \Pi)-1, \\
& i=\operatorname{dim}(\Lambda(\Gamma))=\operatorname{dim}_{H}(\Lambda(\Gamma)), \\
& \Gamma \text { is } i \text {-fuchsian. }
\end{aligned}
$$

Conjecture 10.26. Suppose that $\Gamma$ is a Kleinian group in $\operatorname{Mob}\left(\mathbb{S}^{\mathrm{n}}\right)$ whose limit set is not totally disconnected and has Hausdorff dimension 1 . Then $\Gamma$ is 1-fuchsian.

This conjecture is known to be true for $n=2$, see [50].

Problem 10.27. (The gap problem, L. Bowen, cf. [206].)

1. Is there a number $d_{n}<n$ such that for every Schottky subgroup $\Gamma \subset \operatorname{Mob}\left(\mathbb{S}^{n}\right)$, $n \geq 3$, we have:

$$
\delta(\Gamma)<d_{n}
$$

2. More generally, consider a sequence $\Gamma_{j} \subset \operatorname{Mob}\left(\mathbb{S}^{\mathrm{n}}\right)$ of convex-cocompact groups isomorphic to a fixed group $\Gamma$ so that: $\Lambda\left(\Gamma_{j}\right) \neq \mathbb{S}^{n}$ for each $j$. Is it true that

$$
\lim \sup _{j \rightarrow \infty} \delta\left(\Gamma_{j}\right)<n ?
$$

By the work of R. Phillips and P. Sarnak [185], the answer to the Part 1 of this question is positive in the class of classical Schottky groups.

\section{Representation varieties of Kleinian groups}

For a finitely-generated $\Gamma$ consider the representation variety of $\Gamma$ :

$$
R_{n}(\Gamma):=\operatorname{Hom}\left(\Gamma, \operatorname{Mob}\left(\mathbb{S}^{\mathrm{n}}\right)\right) .
$$

If $\Gamma$ has the presentation

$$
\Gamma=\left\langle x_{1}, \ldots, x_{m} \mid r_{1}, \ldots, r_{k}, \ldots\right\rangle,
$$

the representation variety is given by

$$
\left\{\left(g_{1}, \ldots, g_{m}\right) \in\left(\operatorname{Mob}\left(\mathbb{S}^{n}\right)\right)^{m}: r_{1}\left(g_{1}, \ldots, g_{m}\right)=1, \ldots r_{k}\left(g_{1}, \ldots, g_{m}\right)=1, \ldots\right\} .
$$

The group $\operatorname{Mob}\left(\mathbb{S}^{n}\right)$ acts on $R_{n}(\Gamma)$ via conjugation:

$$
\theta \cdot \rho(\gamma)=\theta \rho(\gamma) \theta^{-1}, \quad \theta \in \operatorname{Mob}\left(\mathbb{S}^{n}\right) .
$$

Given this action, one can form the quotient variety

$$
X_{n}(\Gamma):=R_{n}(\Gamma) / / \operatorname{Mob}\left(\mathbb{S}^{\mathrm{n}}\right),
$$

called the character variety. Roughly speaking, the elements of $X_{n}(\Gamma)$ are represented by conjugacy classes of representations $\rho: \Gamma \rightarrow \operatorname{Mob}\left(\mathbb{S}^{n}\right)$. This is literally true for "most" representations, the ones for which $\rho(\Gamma)$ does not contain a normal parabolic subgroup, see [103]. In general, the representations $\rho_{1}, \rho_{2}$ project to the same point in $X_{n}(\Gamma)$ iff the closures of their $\operatorname{Mob}\left(\mathbb{S}^{n}\right)$-orbits have nonempty intersection. We let $[\rho]$ denote the projection of $\rho \in R_{n}(\Gamma)$ to $X_{n}(\Gamma)$. 
A trivial deformation of a representation $\rho_{0} \in R_{n}(\Gamma)$ is a connected curve $\rho_{t} \in R_{n}(\Gamma)$ which projects to a point in $X_{n}(\Gamma)$. A representation $\rho_{0}$ is called rigid if it admits no nontrivial deformations.

We will be mostly interested in representations $\rho \in R_{n}(\Gamma)$ which have discrete, nonelementary image, however much of our discussion is more general.

In this section we address the following issues related to the character varieties:

(i) Local structure of $X_{n}(\Gamma)$ and existence of small deformations of a given Kleinian group (rigidity vs. flexibility).

(ii) Connectedness of the subspace $\mathcal{D}_{n}(\Gamma)$ of discrete and faithful representations in $X_{n}(\Gamma)$.

(iii) Structural stability: What happens to a Kleinian group in $\mathbf{M o b}\left(\mathbb{S}^{\mathrm{n}}\right)$ if we deform it a little bit? Does it stay Kleinian?

(iv) Compactness of $\mathcal{D}_{n}(\Gamma)$ and estimates on various natural continuous functionals on $\mathcal{D}_{n}(\Gamma)$.

(v) Difficulties in constructing "truly higher-dimensional" geometrically infinite Kleinian groups.

\subsection{Local theory}

We start by considering the local structure of $X_{n}(\Gamma)$. Given an abstract group $\Gamma$ and a representation $\rho \in R_{n}(\Gamma)$, we have the adjoint action of $\rho(\Gamma)$ on the Lie algebra $\mathfrak{g}$ of $\operatorname{Mob}\left(\mathbb{S}^{n}\right)$ and the associated first cohomology group

$$
H^{1}(\Gamma, A d(\rho))=Z^{1}(\Gamma, A d(\rho)) / B^{1}(\Gamma, A d(\rho)),
$$

see Section 4. It was first observed by A. Weil [226] (in the general context of representations to Lie groups) that if $X_{n}(\Gamma)$ is smooth at $[\rho] \in X_{n}(\Gamma)$ then $H^{1}(\Gamma, A d(\rho))$ is isomorphic to the tangent space to $X_{n}(\Gamma)$ at $[\rho]$. Moreover, Weil proved that if $H^{1}(\Gamma, A d(\rho))=0$ then $[\rho]$ is an isolated point on $X_{n}(\Gamma)$, i.e., $\rho$ is rigid.

Therefore, the elements of $H^{1}(\Gamma, A d(\rho))$ can be regarded as infinitesimal deformations of the representation $\rho$. An infinitesimal deformation $[\xi] \in H^{1}(\Gamma, A d(\rho))$ is called integrable if it is tangent to a smooth curve in $X_{n}(\Gamma)$. The obstructions to integrability are cohomological in nature, they are certain elements of $H^{2}(\Gamma, A d(\rho))$, called Massey products. However, in practice, these cohomology classes are very difficult to compute. The first such obstruction is the cup-product:

$$
\phi([\xi])=[\xi] \cup[\xi] \in H^{2}(\Gamma, A d(\rho)),
$$

see for instance [82]. Here $\phi([\xi])$ is represented by the 2-cocycle

$$
\tau(x, y)=[\xi(x), A d \circ \rho(x) \xi(y)],
$$

where [,] is the Lie bracket on the Lie algebra $\mathfrak{g}$. If the first obstruction vanishes and $\Gamma$ is the fundamental group of a surface, then $X_{n}(\Gamma)$ is smooth at $\rho$, see [82], where a much more general result is proved. 
We will be mostly interested in the case where $\rho: \Gamma \hookrightarrow \operatorname{Mob}\left(\mathbb{S}^{n}\right)$ is a discrete embedding, whose image we will identify with $\Gamma$. Then, by abusing the terminology, we will talk of small deformations of $\rho$ in $R_{n}(\Gamma)$ as small deformations of $\Gamma$ itself.

11.1.1. Small deformations of 1-quasifuchsian groups. Recall that $\operatorname{Mob}\left(\mathbb{S}^{n}\right)$ has dimension $d=(n+2)(n+1) / 2$. Suppose that $\Gamma \subset \mathbf{M o b}\left(\mathbb{S}^{n}\right)$ is 1 -quasifuchsian; in this subsection we allow $\Gamma$ to have nontrivial finite order elements. We assume however that $\Gamma$ contains no elements fixing the circle $C=\Lambda(\Gamma)$ pointwise. Therefore we obtain the injective map

$$
\Gamma \rightarrow \operatorname{Isom}\left(\mathbb{H}^{2}\right)
$$

given by the restriction of the elements of $\Gamma$ to the round circle $C$. To simplify the discussion we assume that $\Gamma$ preserves the orientation on $C$. Then $\Gamma$ embeds as a lattice in $P S L(2, \mathbb{R})$.

If $\Gamma$ contains no parabolic elements then it has the presentation: $\left\langle a_{1}, b_{1}, \ldots, a_{q}, b_{q}, c_{1}, \ldots, c_{k} \mid\left[a_{1}, b_{1}\right] \cdots\left[a_{q}, b_{q}\right] \cdot c_{1} \cdots \cdots c_{k}=1, c_{j}^{r_{j}}=1, j=1, \ldots, k\right\rangle$. For a representation $\rho: \Gamma \rightarrow \operatorname{Mob}\left(\mathbb{S}^{n}\right)$ we let

$$
e_{j}:=d-\operatorname{dim}\left\{\xi \in \mathfrak{g}: A d \circ \rho\left(c_{j}\right)(\xi)=\xi\right\} ;
$$

in other words, $e_{j}$ is the codimension of the centralizer of $\rho\left(c_{j}\right)$ in $\operatorname{Mob}\left(\mathbb{S}^{\mathrm{n}}\right)$. Let $s$ denote the dimension of the centralizer of $\rho(\Gamma)$ in $\operatorname{Mob}\left(\mathbb{S}^{n}\right)$.

Theorem 11.1. (A. Weil [226].)

$$
h=\operatorname{dim} H^{1}(\Gamma, A d(\rho))=(2 q-2) d+2 s+e_{1}+\cdots+e_{k} .
$$

Moreover, if $s=0$, then $X_{n}(\Gamma)$ near $[\rho]$ is a smooth $h$-dimensional manifold.

For instance, if $n=1, \Gamma \subset P S L(2, \mathbb{R}) \subset \operatorname{Mob}\left(\mathbb{S}^{1}\right)$; therefore $d=3$, we get $e_{i}=1$ for each $i=1, \ldots, k, s=0$. Hence

$$
h=6 q-6+k,
$$

which is the familiar formula for the dimension of the Teichmüller space of the orbifold $O=\mathbb{H}^{2} / \Gamma$. If $n=2$, we, of course, obtain $h=2(6 q-6+k)$ which is the (real) dimension of the space of the quasifuchsian deformations of $\Gamma$ in $P S L(2, \mathbb{C})$.

To better understand the difficulties which one encounters in the case of $i$-fuchsian groups for $i \geq 2$, we consider the hyperbolic triangle groups $\Gamma$. The reason for considering these groups is that they are rigid in $\operatorname{PSL}(2, \mathbb{R})$ (similarly to rigidity of lattices in $\left.\operatorname{Mob}\left(\mathbb{S}^{n}\right), n \geq 2\right)$.

The triangle groups are the 1 -fuchsian groups with $q=0, k=3$; every such $\Gamma$ has the presentation

$$
\left\langle c_{1}, c_{2}, c_{3} \mid c_{1} \cdot c_{2} \cdot c_{3}=1, c_{j}^{r_{j}}=1, j=1,2,3\right\rangle,
$$

where $r_{1}^{-1}+r_{2}^{-1}+r_{3}^{-1}<1$. Such group embeds discretely into $P S L(2, \mathbb{R})$ and we will denote the image of this embedding by $\Delta=\Delta\left(r_{1}, r_{2}, r_{3}\right)$

As a subgroup of $\mathbf{M o b}\left(\mathbb{S}^{2}\right)$, the group $\Delta$ is rigid (which follows from vanishing of $\left.H^{1}\right)$. Moreover, triangle groups are "strongly rigid" in $\operatorname{Mob}\left(\mathbb{S}^{2}\right)$, i.e., every 
discrete embedding of $\Gamma$ into $\operatorname{Mob}\left(\mathbb{S}^{2}\right)$ is induced by conjugation of the identity embedding, see [84] for the complete description of $X_{2}(\Gamma)$.

The situation changes somewhat if we consider representations into $\operatorname{Mob}\left(\mathbb{S}^{3}\right)$. First, let $\rho_{0}: \Delta \rightarrow \Gamma^{\prime} \subset \operatorname{Mob}\left(\mathbb{S}^{3}\right)$ be the embedding obtained as the composition

$$
\Delta \subset \operatorname{Mob}\left(\mathbb{S}^{1}\right) \hookrightarrow \operatorname{Mob}\left(\mathbb{S}^{2}\right) \hookrightarrow \operatorname{Mob}\left(\mathbb{S}^{3}\right)
$$

of natural embeddings. Then $\operatorname{dim} H^{1}\left(\Delta, A d\left(\rho_{0}\right)\right)=0$ and hence $\rho_{0}$ is still rigid in $\operatorname{Mob}\left(\mathbb{S}^{3}\right)$. The easiest way to see this is to use Weil's formula (11.1):

$$
d=10, s=1, e_{j}=6, \quad \text { for } j=1,2,3
$$

and hence

$$
h=-20+2+6+6+6=0 .
$$

However, instead of $\rho_{0}$ we can take a twisted extension. Suppose that we can find numbers $m_{j} \in \mathbb{Z}, 1<\left|m_{j}\right|<r_{j}-1(j=1,2,3)$ such that:

$$
m_{1}^{-1}+m_{2}^{-1}+m_{3}^{-1}=0, \text { and } \forall j, m_{j} \text { divides } r_{j} .
$$

(This is satisfied for instance by $m_{1}=m_{2}=4, m_{3}=-2$ and $r_{j}=8$ for all $j$.)

Define a homomorphism $\theta: \Delta \rightarrow S O(2)$ by sending $c_{i}$ to the rotation by $2 \pi / m_{i}$. Then define $\rho: \Delta \rightarrow \operatorname{Mob}\left(\mathbb{S}^{1}\right) \times S O(2) \subset \operatorname{Mob}\left(\mathbb{S}^{3}\right)$ by twisting $\rho_{0}$ via $\theta$ :

$$
\rho(\gamma)=\rho_{0}(\gamma) \times \theta(\gamma), \quad \gamma \in \Delta
$$

It is clear that $\rho(\Delta)$ is again a 1-fuchsian subgroup in $\operatorname{Mob}\left(\mathbb{S}^{3}\right)$. If $r_{j}>3$ for each $j$, then $e_{j}=8, s=1$ and the formula (11.1) gives the dimension $h=6$ for $H^{1}(\Delta, A d(\rho))$. I do not know if any of these infinitesimal deformations is integrable. To decide this one has to analyze the quadratic form

$$
\phi: H^{1}(\Delta, A d(\rho)) \rightarrow H^{2}(\Delta, A d(\rho))=\mathbb{R},
$$

given by the cup-product. According to [82], the quadratic cone $\{\phi=0\}$ is analytically isomorphic to a neighborhood of $[\rho]$ in $X_{3}(\Delta)$; hence it suffices to find a nontrivial 1-cocycle $\xi$ for which $\phi([\xi])=0$ to get nontrivial deformations of the representation $\rho$.

On the other hand, one can use (11.1) to show that every representation $\rho$ of the group $\Delta=\Delta\left(2,3, r_{3}\right)$ into $\mathbf{M o b}\left(\mathbb{S}^{3}\right)$ has zero cohomology $H^{1}(\Delta, A d(\rho))$. Therefore $X_{3}(\Delta)$ is a zero-dimensional algebraic variety and, hence, is a finite set.

This situation is somewhat typical for representations of lattices in $\operatorname{Mob}\left(\mathbb{S}^{n}\right)$ $(n \geq 2)$ into $\operatorname{Mob}\left(\mathbb{S}^{n+1}\right)$ : In a number of cases we can prove rigidity by making cohomological computations; in some cases we can only conclude that $H^{1}$ is nonzero, without being able to make a definitive conclusion about existence of nontrivial deformations. 
11.1.2. Small deformations of $i$-quasifuchsian groups for $i \geq 2$. In the case of $(n-1)$-quasifuchsian groups $\Gamma(n \geq 2)$, the existence of nontrivial deformations of $\Gamma$ in $\operatorname{Mob}\left(\mathbb{S}^{n}\right)$ is not at all clear. Suppose that $\Gamma \subset \operatorname{Isom}\left(\mathbb{H}^{n}\right)$ is a cocompact lattice. Then the identity embedding

$$
\iota: \Gamma \hookrightarrow \operatorname{Isom}\left(\mathbb{H}^{n}\right),
$$

is rigid by Mostow's theorem.

Remark 11.2. Actually, (local) rigidity of $\iota$ was known prior to the work of Mostow; it was first established by E. Calabi [48], whose proof was later generalized by A. Weil $[224,225]$. These arguments were based on proving that $H^{1}(\Gamma, A d(\iota))=0$.

Consider now the composition of $\iota$ with the natural embedding:

$$
\rho: \Gamma \rightarrow \operatorname{Isom}\left(\mathbb{H}^{n}\right) \hookrightarrow \operatorname{Isom}\left(\mathbb{H}^{n+1}\right) .
$$

Then

$$
H^{1}(\Gamma, A d(\iota)) \cong H^{1}\left(\Gamma, V_{n}\right),
$$

where $V_{n}=\mathbb{R}^{n, 1}$ and $\Gamma$ acts on the Lorentz space $\mathbb{R}^{n, 1}$ via the usual embedding $\Gamma \hookrightarrow O(n, 1)$.

It turns out that $\rho$ may or may not be rigid, even if $\Gamma$ is torsion-free: Rigidity depends on the lattice $\Gamma$. One has the following list ((a) through (e)) of constructions of deformations and infinitesimal deformations of $[\rho]$ in $X_{n}(\Gamma)$ :

(a) Bending, see [103], [135]. Given a connected properly embedded totallygeodesic hypersurface $S \subset M=\mathbb{H}^{n} / \Gamma$, one associates with $S$ a smooth curve through $[\rho]$ in $X_{n}(\Gamma)$, called the bending deformation of $[\rho]$. More generally, given a disjoint collections of such hypersurfaces $S_{1}, \ldots, S_{k}$, one obtains a $k$-dimensional smooth submanifold in $X_{n}(\Gamma)$ containing $[\rho]$. This construction is completely analogous to bending deformations of 1-fuchsian subgroups in $\operatorname{Mob}\left(\mathbb{S}^{2}\right)$ defined by W. Thurston in [213]. We let $\left[\xi_{S}\right]$ denote the element of $H^{1}(\Gamma, A d(\rho))$ corresponding to a connected totally-geodesic hypersurface $S \subset M$.

There are numerous groups $\Gamma$ satisfying assumptions of the bending construction. Namely, start with an arithmetic group $O^{\prime}(f, A)$ of the simplest type (see Section 2), where

$$
f=a_{0} x_{0}^{2}+a_{1} x_{1}^{2}+\cdots+a_{n} x_{n}^{2},
$$

and $a_{0}<0, a_{i}>0, i=1, \ldots, n$. Identify $\mathbb{H}^{n}$ with a component of the hyperboloid $\{f(x)=-1\}$. Then the stabilizer of the hyperplane $P=\left\{x_{n}=0\right\}$ in $O^{\prime}(f, A)$ is an arithmetic lattice in $\operatorname{Isom}\left(\mathbb{H}^{n-1}\right)$. The intersection $H=P \cap \mathbb{H}^{n}$ is a hyperplane in $\mathbb{H}^{n}$. After passing to an appropriate finite-index subgroup $\Gamma$ in $O^{\prime}(f, A)$, one obtains a totally-geodesic embedding of the hypersurface $S=H / \Gamma^{\prime}$ into $H / \Gamma$, where $\Gamma^{\prime}=\Gamma \cap O^{\prime}(f, A)$. We refer the reader to [164] for the details.

Problem 11.3 (I. Rivin). Construct examples of hyperbolic $n$-manifolds $M$ of finite volume $(n \geq 4)$ such that $M$ contains a separating properly embedded totallygeodesic hypersurface $S \subset M$. Note that the main objective of [164] was to construct nonseparating hypersurfaces. 
The idea of bending deformations of representations is quite simple and has nothing to do with the hyperbolic space. Below is a general description of bending as defined by D. Johnson and J. Millson in [103]. Suppose that we are given a graph of groups $\mathcal{G}$ with the vertex groups $\Gamma_{v}$ and the edge groups $\Gamma_{e}$. Let $\Gamma=\pi_{1}(\mathcal{G})$ be the fundamental group of $\mathcal{G}$. For instance, the amalgam

$$
\Gamma=\Gamma_{v_{1}} *_{\Gamma_{e}} \Gamma_{v_{2}}
$$

is the fundamental group of a graph of groups which is a single edge $e$ with two vertices $v_{1}, v_{2}$. Let $\rho_{0}: \Gamma \rightarrow G$ be a representation of $\Gamma$ to a Lie group $G$. A bending deformation of $\rho_{0}$ is a curve of representations $\rho_{t}: \Gamma \rightarrow G, t \in[-1,1]$, such that for each vertex group $\Gamma_{v}$, we have

$$
\rho_{t} \mid \Gamma_{v}=g_{v, t}\left(\rho_{0} \mid \Gamma_{v}\right) g_{v, t}^{-1},
$$

for some curve $g_{v, t}$ of elements of $G$.

Therefore, the restriction of $\rho_{t}$ to each vertex group determines a trivial deformation of the representation of this group. The trick is that the deformation of the representation of the entire group $\Gamma$ may be still nontrivial. For instance, in the case of the amalgam (11.2), let $g_{t} \in G, g_{0}=1$, be a curve of elements centralizing $\rho\left(\Gamma_{e}\right)$, but not $\rho\left(\Gamma_{v_{1}}\right), \rho\left(\Gamma_{v_{2}}\right)$. Define the family of representations

$$
\rho_{t}: \Gamma \rightarrow G, \quad \rho_{t}\left|\Gamma_{v_{1}}=\rho_{0}\right| \Gamma_{v_{1}}, \quad \rho_{t} \mid \Gamma_{v_{2}}=g_{t}\left(\rho_{0} \mid \Gamma_{v_{1}}\right) g_{t}^{-1} .
$$

In the case of the HNN extension

$$
\Gamma=\Gamma_{v_{1}} *_{e}
$$

generated by $\Gamma_{v_{1}}$ and $\tau \in \Gamma \operatorname{such} \tau \Gamma_{e} \tau^{-1}=\Gamma_{e}^{\prime} \subset \Gamma_{v_{1}}$, we take

$$
\rho_{t}\left|\Gamma_{v_{1}}=\rho_{0}\right| \Gamma_{v_{1}}, \quad \rho_{t}(\tau)=\rho_{0}(\tau) g_{t} .
$$

This is a nontrivial deformation of the representation $\rho_{0}$.

We now return to the case when $\Gamma=\pi_{1}(M), M$ is a hyperbolic $n$-manifold containing pairwise disjoint totally geodesic hypersurfaces $S_{i}, i=1, \ldots, k$. Then the group $\Gamma$ splits as the graph of groups $\mathcal{G}$, so that the vertex subgroups $\Gamma_{v_{j}}$ are the fundamental groups of the components of $M \backslash\left(S_{1} \cup \cdots \cup S_{k}\right)$ and the edge groups $\Gamma_{e_{i}}$ are the fundamental groups $\pi_{1}\left(S_{i}\right)$. Therefore

1. The centralizer of each $\Gamma_{e_{i}}=\pi_{1}\left(S_{i}\right)$ in $\operatorname{Mob}\left(\mathbb{S}^{n}\right)$ is 1-dimensional (the group of elliptic rotations around the limit set of $\Gamma_{e_{i}}$ ).

2. The centralizer in $\operatorname{Mob}\left(\mathbb{S}^{n}\right)$ of the fundamental group of each $\Gamma_{v_{j}}$ is zerodimensional (i.e., $\mathbb{Z}_{2}$ ).

Hence one obtains nontrivial bending deformations $\rho_{t}$ of the identity embedding of $\rho: \Gamma \hookrightarrow \operatorname{Mob}\left(\mathbb{S}^{n}\right)$. The set of bending parameters $t=\left(t_{1}, \ldots, t_{k}\right)$ can be identified with $\left(\mathbb{S}^{1}\right)^{k}$, as the centralizer of each $\Gamma_{e_{i}}$ in $\operatorname{Mob}\left(\mathbb{S}^{n}\right)$ is the circle $S O(2)$.

Theorem 11.4. (D. Johnson and J. Millson [103].) For every $n \geq 4$ there exists a uniform lattice $\Gamma \subset \operatorname{Isom}\left(\mathbb{H}^{n}\right)$ and intersecting hypersurfaces $S_{1}, S_{2} \subset \mathbb{H}^{n} / \Gamma$, so that

$$
\left[\xi_{S_{1}}\right] \cup\left[\xi_{S_{2}}\right] \in H^{2}(\Gamma, A d(\rho)) \neq 0 .
$$


In particular, $X_{n}(\Gamma)$ is not smooth at $[\rho]$.

In contrast with this result, the character varieties $X_{2}(\Gamma)$ tend to be smooth:

Theorem 11.5. (M. Kapovich [116, Theorem 8.44].) Let $\Gamma \subset \operatorname{Mob}\left(\mathbb{S}^{2}\right)$ be a discrete subgroup. Then the identity embedding $\iota: \Gamma \rightarrow \operatorname{Mob}\left(\mathbb{S}^{2}\right)$ determines a smooth point on $X_{2}(\Gamma)$.

On the other hand, there are cocompact lattices $\Gamma \subset \operatorname{Mob}\left(\mathbb{S}^{2}\right)$ and (nondiscrete) representations $\rho: \Gamma \rightarrow \operatorname{Mob}\left(\mathbb{S}^{2}\right)$ for which $X_{2}(\Gamma)$ has nonquadratic singularity at $[\rho]$, see $[126]$.

(b) Generalized bending associated with a collection of compact totally-geodesic submanifolds with boundary in $M^{n}$, see [12] ${ }^{8}$, [125], [161], [13].

The idea of the generalized bending is that instead of considering fundamental groups of graphs of groups, one looks at the more general complexes of groups. The only examples which had been worked out are 2-dimensional complexes of groups. Let $\mathcal{X}$ be such a complex with the vertex groups $\Gamma_{v}$. Let $\pi_{1}(\mathcal{X})=\Gamma$ and $\rho_{0}: \Gamma \rightarrow G$ be a representation to a Lie group. Then, as in the definition of bending, a generalized bending of $\rho_{0}$ is a curve of representations $\rho_{t}: \Gamma \rightarrow G, t \in[-1,1]$, whose restrictions to each vertex subgroup $\Gamma_{v}$ are trivial deformations of $\rho_{0} \mid \Gamma_{v}$.

(c) Suppose that a lattice $\Gamma \subset \operatorname{Isom}\left(\mathbb{H}^{n}\right)$ is a reflection group, i.e., it is generated by reflections in the faces of a convex acute polyhedron $\Phi \subset \mathbb{H}^{n}$ of finite volume (the fundamental domain of $\Gamma$ ). If $f$ is the number of facets of $\Phi$, then one can show that

$$
\operatorname{dim} H^{1}(\Gamma, A d(\rho))=f-n-1,
$$

see [113]. The facets of $\Phi$ correspond to vectors spanning $H^{1}$. If $n \geq 4$, it is unclear which (if any) of these infinitesimal deformations are integrable. Of course, in some examples some of these infinitesimal deformations are integrable, since they appear as infinitesimal bending deformations. If $n=3$, then $X_{3}(\Gamma)$ is smooth near $[\rho]$ and has dimension $f-4$, see [113].

And that's all for $n \geq 3$.

Problem 11.6 (P. Storm). Let $M$ be a compact hyperbolic $(n+1)$-dimensional manifold with nonempty totally-geodesic boundary, $n \geq 3$. Let $\Gamma:=\pi_{1}(M) \subset$ $\operatorname{Mob}\left(\mathbb{S}^{\mathrm{n}}\right)$. Is it true that $\Gamma$ is rigid in $\operatorname{Mob}\left(\mathbb{S}^{\mathrm{n}}\right)$ ?

Note that (by Mostow rigidity) rigidity of $\Gamma$ would follow if we knew that for each component $S$ of $\partial M$, the fundamental group $\Gamma_{S}:=\pi_{1}(S)$ is rigid in $\mathbf{M o b}\left(\mathbb{S}^{n}\right)$. At the moment, we do not have results in either direction of this problem:

1. It is unclear if any of the rigid hyperbolic 3-manifolds, or their disjoint union (see Example 11.1.2), bounds a compact hyperbolic 4-manifold.

2. Even if some $\Gamma_{S}$ is not rigid, it is unclear if its deformations extend to deformations of $\Gamma$.

${ }^{8}$ Some of the theorems stated in this paper are probably incorrect since they do not take into account the restrictions on the angles between the totally-geodesic submanifolds. 
The only known example of a rigid group $\Gamma$ (as in Problem 11.6) is the fundamental group of a 4-dimensional hyperbolic orbifold. Moreover, in this example the group $\Gamma_{S}$ is not rigid:

Consider the 120-cell $D^{4} \subset \mathbb{H}^{4}$ which appears in [60]. Pick a facet $F \subset D^{4}$. Let $\Gamma \subset \operatorname{Mob}\left(\mathbb{S}^{3}\right)$ be the Kleinian group generated by reflections in all facets of $D^{4}$ except for $F$. Then $\Gamma$ is the fundamental group of a right-angled 4-dimensional reflection orbifold $\mathcal{O}$ with boundary (the boundary $S=\partial \mathcal{O}$ corresponds to the facet $F$ ). The subgroup $\Gamma_{S}:=\pi_{1}(S)$ is the Coxeter group generated by reflections in the facets of the regular right-angled hyperbolic dodecahedron. In particular, $X_{3}(\Gamma)$ is a smooth 8-dimensional manifold near $[\iota]$, where $\iota: \Gamma_{S} \hookrightarrow \operatorname{Mob}\left(\mathbb{S}^{2}\right) \hookrightarrow$ $\operatorname{Mob}\left(\mathbb{S}^{3}\right)$ is the identity embedding.

Theorem 11.7 (M. Kapovich). $\Gamma$ is rigid in $\operatorname{Mob}\left(\mathbb{S}^{3}\right)$.

Assume now that $n=3$.

(d) The first obstruction to integrability of infinitesimal deformations is always zero, see [126].

Question 11.8. Suppose that $\Gamma \subset \operatorname{Mob}\left(\mathbb{S}^{2}\right)$ is a cocompact lattice. It it true that the character variety $X_{3}(\Gamma)$ is smooth at the point $[\rho]$ ?

(e) Finally, there are several constructions which work for specific examples of lattices $\Gamma \subset \operatorname{Mob}\left(\mathbb{S}^{2}\right)$, e.g., stumping deformations [9], generalized in [212].

We recall the following

Conjecture 11.9. Suppose that $\Gamma \subset \operatorname{Mob}\left(\mathbb{S}^{n}\right)$ is a lattice. Then $\Gamma$ contains a finiteindex subgroup $\Gamma^{\prime}$ such that $\Gamma^{\prime}$ has infinite abelianization, i.e., $H^{1}\left(\Gamma^{\prime}, \mathbb{R}\right) \neq 0$.

We refer the reader to $[144,146,164,189,200]$ for various results towards this conjecture in the case of arithmetic lattices in $\operatorname{Isom}\left(\mathbb{H}^{n}\right)$. The methods used in these papers for proving virtual nonvanishing of the first cohomology group usually also apply to the cohomology groups $H^{1}(\Gamma, A d(\rho))$, where $\rho: \Gamma \rightarrow \operatorname{Isom}\left(\mathbb{H}^{n+1}\right)$ is the natural embedding. On the other hand, the proofs of special cases of Conjecture 11.9 for hyperbolic 3-manifolds which use the methods of 3-dimensional topology (see, e.g., [142]), usually provide no information about rigidity of $\Gamma$ in $\operatorname{Isom}\left(\mathbb{H}^{4}\right)$.

Conjecture 11.10. Suppose that $\Gamma \subset \operatorname{Isom}\left(\mathbb{H}^{n}\right)$ is a lattice. Then there exists a finite-index subgroup $\Gamma^{\prime} \subset \Gamma$ so that $H^{1}\left(\Gamma^{\prime}, A d(\rho)\right) \neq 0$.

On the other hand, some uniform torsion-free lattices in $\operatorname{Mob}\left(\mathbb{S}^{2}\right)$ are rigid in $\operatorname{Mob}\left(\mathbb{S}^{3}\right)$ :

Example. In [113] we constructed examples of (torsion-free) cocompact lattices $\Gamma$ in $\operatorname{Mob}\left(\mathbb{S}^{2}\right)$ for which $H^{1}(\Gamma, A d(\rho))=0$, where $\rho: \Gamma \rightarrow \operatorname{Mob}\left(\mathbb{S}^{3}\right)$ is the natural embedding. The quotient manifolds $\mathbb{H}^{3} / \Gamma$ in these examples are non-Haken. K. Scannell [197] constructed analogous examples with Haken quotients $\mathbb{H}^{3} / \Gamma$. 
More specifically, it was proved in [113] that for every hyperbolic 2-bridge knot $K \subset \mathbb{S}^{3}$, there are infinitely many (hyperbolic) Dehn surgeries on $K$, so that for the resulting manifolds $M_{j}, j \in \mathbb{N}$, we have

$$
H^{1}\left(\Gamma_{j}, A d(\rho)\right)=0, \text { where } \Gamma_{j}=\pi_{1}\left(M_{j}\right) .
$$

11.1.3. Failure of quasiconformal isotopy. The goal of this section is to construct examples of Kleinian groups which are quasiconformally conjugate, but cannot be deformed to each other. As the reader will see, the tools for constructing such examples were available 12 years ago. I realized that such examples exist only recently, while working on this survey.

Theorem 11.11. There exists a pair of convex-cocompact Kleinian groups $\Delta_{1}, \Delta_{2} \subset$ $\operatorname{Mob}\left(\mathbb{S}^{5}\right)$ and a quasiconformal homeomorphism $f: \mathbb{S}^{5} \rightarrow \mathbb{S}^{5}$ conjugating $\Delta_{1}$ to $\Delta_{2}$, which is not isotopic to the identity through homeomorphisms $h_{t}: \mathbb{S}^{5} \rightarrow \mathbb{S}^{5}$ such that

$$
h_{t} \Delta_{1} h_{t}^{-1} \subset \operatorname{Mob}\left(\mathbb{S}^{5}\right) .
$$

Proof. We begin with a lattice $\Gamma=\pi_{1}(N)$, where $N=M_{j}$ is as in the discussion of Example 11.1.2 and $K \subset \mathbb{S}^{3}$ is the figure 8 knot. Consider the representation

$$
\rho_{1}: \Gamma \hookrightarrow \operatorname{Mob}\left(\mathbb{S}^{2}\right) \hookrightarrow \operatorname{Mob}\left(\mathbb{S}^{5}\right)
$$

obtained by the composition of natural embeddings. Then

$$
H^{1}\left(\Gamma, A d\left(\rho_{1}\right)\right)=H^{1}\left(\Gamma, V_{3} \oplus V_{3} \oplus V_{3} \oplus \mathbb{R}^{3}\right),
$$

where $V_{3}=\mathbb{R}^{3,1}$ and $\mathbb{R}^{3}$ is the trivial 3-dimensional $\mathbb{R} \Gamma$-module. Since $H^{1}\left(\Gamma, V_{3}\right)=$ 0 by [113] and $H^{1}\left(\Gamma, \mathbb{R}^{3}\right)=0$ since $N$ is a rational homology sphere, we obtain

$$
H^{1}\left(\Gamma, A d\left(\rho_{1}\right)\right)=0 .
$$

Therefore $\rho_{1}$ is rigid. If $N$ is an integer homology 3-sphere, then nonvanishing of the Casson invariant of $K$ implies that $\Gamma$ admits a nontrivial homomorphism

$$
\theta: \Gamma \rightarrow S O(3),
$$

which lifts to $S U(2)$, see [7]. If $M_{j}$ is not an integer homology sphere, then $\Gamma$ has nontrivial abelianization and hence we also obtain a nontrivial homomorphism $\theta: \Gamma \rightarrow S O(3)$ with cyclic image. In any case, we twist the representation $\rho_{1}$ by $\theta$ :

$$
\rho_{2}=\rho_{1} \times \theta: \Gamma \rightarrow \operatorname{Mob}\left(\mathbb{S}^{2}\right) \times S O(3) \subset \operatorname{Mob}\left(\mathbb{S}^{5}\right) .
$$

It is clear that $\left[\rho_{1}\right],\left[\rho_{2}\right]$ are distinct points of $X_{5}(\Gamma)$. The images of $\rho_{1}$ and $\rho_{2}$ are 2 -fuchsian, convex-cocompact groups $\Delta_{1}, \Delta_{2} \subset \operatorname{Mob}\left(\mathbb{S}^{5}\right)$. We obtain the isomorphism

Clearly,

$$
\rho:=\rho_{2} \circ \rho_{1}^{-1}: \Delta_{1} \rightarrow \Delta_{2}
$$

$$
M^{5}\left(\Delta_{1}\right)=N \times \mathbb{S}^{2}
$$

while $M^{5}\left(\Delta_{2}\right)$ is the 2 -sphere bundle over $N$ associated with $\theta$. It is easy to see that the latter bundle is (smoothly) trivial. Therefore we obtain a diffeomorphism

$$
h: M^{5}\left(\Delta_{1}\right) \rightarrow M^{5}\left(\Delta_{2}\right)
$$


which lifts to a $\rho$-equivariant diffeomorphism $f: \Omega\left(\Delta_{1}\right) \rightarrow \Omega\left(\Delta_{2}\right)$. The latter extends to a quasiconformal homeomorphism $f: \mathbb{S}^{5} \rightarrow \mathbb{S}^{5}$ by Theorem 6.1. If there was a continuous family of homomorphisms $\rho_{t}$ connecting $\rho$ to the identity embedding $\Delta_{1} \rightarrow \Delta_{2}$, then the representation $\rho_{1}$ would not be rigid in $X_{5}(\Gamma)$. Contradiction.

By embedding naturally the groups $\Delta_{1}, \Delta_{2}$ to $\operatorname{Mob}\left(\mathbb{S}^{\mathrm{n}}\right)$ for $n \geq 6$ one obtains higher-dimensional examples.

\subsection{Stability theorem}

Let $\Gamma \subset \operatorname{Mob}\left(\mathbb{S}^{\mathrm{n}}\right)$ be a geometrically finite Kleinian group. Consider the set of cusps in $\Gamma$ :

$$
\left[\Pi_{1}\right], \ldots,\left[\Pi_{m}\right],
$$

where $\Pi_{i}$ are maximal parabolic subgroups of $\Gamma$. We define the (topologically) relative representation variety

$$
R_{n}^{t o p}(\Gamma)=\left\{\rho: \Gamma \rightarrow \operatorname{Mob}\left(\mathbb{S}^{n}\right): \rho\left(\Pi_{i}\right) \text { is topologically conjugate to } \Pi_{i} \text { in } \mathbb{S}^{n}, \forall i\right\}
$$

and the (quasiconformally) relative representation variety

$$
R_{n}^{q c}(\Gamma)=\left\{\rho: \Gamma \rightarrow \operatorname{Mob}\left(\mathbb{S}^{n}\right): \rho\left(\Pi_{i}\right) \text { is quasiconformally conjugate to } \Pi_{i} \text { in } \mathbb{S}^{n}\right\} .
$$

Let Homeo $\left(\mathbb{S}^{n}\right)$ and $Q C\left(\mathbb{S}^{n}\right)$ be the groups of homeomorphisms and quasiconformal homeomorphisms of $\mathbb{S}^{n}$ with the topology of uniform convergence. Let $X_{n}^{t o p}(\Gamma), X_{n}^{q c}(\Gamma)$ be the projections of $R_{n}^{t o p}(\Gamma), R_{n}^{q c}(\Gamma)$ to $X_{n}(\Gamma)$. Let $\iota: \Gamma \rightarrow$ $\operatorname{Mob}\left(\mathbb{S}^{n}\right)$ be the identity embedding. Then the Stability Theorem for geometrically finite groups states that every homomorphism $\rho$ of $\Gamma$ sufficiently close to $\iota$ is induced by a (quasiconformal) homeomorphism $h_{\rho}$ close to the identity and depending continuously on $\rho$. More precisely:

Theorem 11.12. (Stability theorem, see $[40,69,79,108,152,210]$.) There exist neighborhoods $U^{\text {top }}, U^{q c}$ of $\iota$ in $R_{n}^{\text {top }}(\Gamma), R_{n}^{q c}(\Gamma)$ respectively, and continuous maps

$$
L^{\text {top }}: U^{\text {top }} \rightarrow \operatorname{Homeo}\left(\mathbb{S}^{n}\right), \quad L^{q c}: U^{q c} \rightarrow Q C\left(\mathbb{S}^{n}\right)
$$

so that

$$
L^{t o p}(\iota)=L^{q c}(\iota)=I d,
$$

and for every $\rho \in U^{\text {top }}$, resp. $\rho \in U^{q c}$, the homeomorphism $L^{\text {top }}(\rho)$, resp. $L^{q c}(\rho)$ is $\rho$-equivariant.

This theorem was first proved by A. Marden in [152] in the case $n=3$. Marden was using convex finitely-sided fundamental domains with simplicial links of vertices: Such polyhedra are generic among the Dirichlet fundamental domains, see [106]. Marden then argued that a small perturbation of such fundamental domain is again a fundamental domain (by the Poincaré fundamental polyhedron theorem). Moreover, the simplicial assumption implies that the combinatorics of the fundamental domain does not change under a small perturbation. This allowed Marden to construct an equivariant quasiconformal homeomorphism close to the identity. This argument does not readily generalize to higher dimensions, mainly 
because finiteness of the number of faces is not equivalent to geometric finiteness. (Otherwise, the same argument goes through.)

D. Sullivan [210] considered the case of general $n$, but assumed that $\Gamma$ is convex-cocompact. Then he proved the existence of a homeomorphism $h_{\rho}$ defined on the limit set of $\Gamma$ and the fact that it depends continuously on $\rho$. The fact that

$$
h_{\rho}: \Lambda(\Gamma) \rightarrow \Lambda(\rho(\Gamma))
$$

is necessarily quasi-symmetric, then follows from Tukia's theorem 6.1 . One then has to show existence of a $\rho$-equivariant diffeomorphism of the domains of discontinuity

$$
f_{\rho}: \Omega(\Gamma) \rightarrow \Omega(\rho(\Gamma))
$$

smoothly depending on $\rho$. This is achieved by appealing to Thurston's holonomy theorem (see $[69,79]$ ) for the Möbius structures on the manifold $M^{n}(\Gamma)$, as it is done in $[101,108]$. The homeomorphisms $h_{\rho}$ and $f_{\rho}$ yield a $\rho$-equivariant quasiconformal homeomorphism of the $n$-sphere by Theorem 6.1 .

The proof in [69] is a good alternative to the above argument; it is also sufficiently flexible to handle the case of geometrically finite Kleinian groups with cusps. Namely, instead of working with the $n$-dimensional manifold $M^{n}(\Gamma)$ one works with the convex hyperbolic $(n+1)$-manifold

$$
H(\Gamma):=\operatorname{Hull}_{\epsilon}(\Lambda(\Gamma)) / \Gamma .
$$

An analogue of Thurston's holonomy theorem for manifolds with boundary applies in this case. Thus, for $\rho \in U^{t o p}$, there exists a hyperbolic structure $s(\rho)$ (with the holonomy $\rho$ ) on the thick part

$$
H(\Gamma)_{[\mu, \infty)}
$$

of the manifold $H(\Gamma)$. Moreover, convexity of the boundary for the new hyperbolic structures (away from the cusps) persists under small perturbations of the hyperbolic structure. Therefore, if $\Gamma$ is convex-cocompact, $\Gamma^{\prime}:=\rho(\Gamma)$ is again convex-cocompact and $\rho: \Gamma \rightarrow \Gamma^{\prime}$ is an isomorphism. If $\Gamma$ is merely geometrically finite, because $\rho$ belongs to the relative representation variety, it follows that the hyperbolic structure $s(\rho)$ extends to a convex complete hyperbolic structure on the cusps. This argument also yields a $\rho$-equivariant diffeomorphism

$$
\operatorname{Hull}_{\epsilon}(\Lambda(\Gamma)) \rightarrow \operatorname{Hull}_{\epsilon}\left(\Lambda\left(\Gamma^{\prime}\right)\right)
$$

depending continuously on $\rho$. To get from the convex hulls to the domain of discontinuity one uses the existence of the canonical equivariant diffeomorphisms ("the nearest-point projections")

$$
\Omega(\Gamma) \rightarrow \partial H u l l_{\epsilon}(\Lambda(\Gamma)), \quad \Omega\left(\Gamma^{\prime}\right) \rightarrow \partial H u l l_{\epsilon}\left(\Lambda\left(\Gamma^{\prime}\right)\right) .
$$

We refer the reader to [69] for the details.

Sullivan also had a converse to the Stability Theorem for (finitely-generated) subgroups on $\operatorname{Mob}\left(\mathbb{S}^{2}\right)$ : 
Theorem 11.13. (D. Sullivan, [210, Theorem A']) If a (finitely-generated) Kleinian subgroup of $\operatorname{Mob}\left(\mathbb{S}^{2}\right)$ is stable in the sense of Theorem 11.12 , then it is geometrically finite or its identity embedding in $\operatorname{Mob}\left(\mathbb{S}^{2}\right)$ is rigid in $X_{2}(\Gamma)$.

It was proved in [116] that every rigid $\Gamma$ in the above theorem has to be geometrically finite. Now it, of course, follows from the positive solution of the Bers-Thurston density conjecture (geometrically finite groups are dense among Kleinian subgroups of $\operatorname{Mob}\left(\mathbb{S}^{2}\right)$ ).

Question 11.14. Does Theorem 11.13 hold for subgroups of $\mathbf{M o b}\left(\mathbb{S}^{n}\right), n \geq 3$ ?

We expect the answer to be negative.

\subsection{Space of discrete and faithful representations}

Let $\mathcal{D}_{n}(\Gamma) \subset X_{n}(\Gamma)$ denote the subset corresponding to discrete, injective and nonelementary representations of $\Gamma$.

Theorem 11.15 (Chuckrow-Jørgensen-Wielenberg). $\mathcal{D}_{n}(\Gamma) \subset X_{n}(\Gamma)$ is closed. See for instance $[227,154]$.

It turns out that there exists another way to construct limits of sequences of Kleinian groups, by regarding them as closed subsets of $\mathbf{M o b}\left(\mathbb{S}^{\mathrm{n}}\right)$. This leads to the topology of geometric convergence of Kleinian groups. With few exceptions, the space of Kleinian groups is again closed in this topology (see, e.g., $[215,116]$ ). In general, $\mathcal{D}_{n}(\Gamma)$ is not compact. Nevertheless, this space can be compactified by projective classes of nontrivial $\Gamma$-actions on real trees. This compactification generalizes Thurston's compactification of the Teichmüller space. The compactification by actions on trees was first defined by J. Morgan and P. Shalen [172] and J. Morgan [171] using algebraic geometry. More flexible, geometric, definitions of this compactification were introduced by M. Bestvina [23] and F. Paulin [182]. See also [116] for the construction of this compactification using ultralimits of metric spaces.

This viewpoint provides a powerful tool for proving compactness of $\mathcal{D}_{n}(\Gamma)$ for certain classes of groups: If $\mathcal{D}_{n}(\Gamma)$ is non-compact then $\Gamma$ admits nontrivial action on a certain $\mathbb{R}$-tree. One then proves that such action cannot exist. The tools for proving such non-existence theorems are originally due to Morgan and Shalen (but limited to the fundamental groups of 3-manifolds, see [172]); a much more general method is due to E. Rips (Rips theory), see [27]. One then obtains the following (see, e.g., [116]):

Theorem 11.16 (Rips-Thurston Compactness theorem). Suppose that $\Gamma$ is a finitely-presented group which does not split as an amalgam over a virtually abelian group. Then $\mathcal{D}_{n}(\Gamma)$ is compact.

Remark 11.17. W. Thurston [214] proved this theorem for a certain class of 3manifold groups in the case $n=2$.

Unfortunately, none of the known proofs of Theorem 11.16 gives an explicit bound on the "size" of $\mathcal{D}_{n}(\Gamma)$. 
Problem 11.18. Find a "constructive" proof of Theorem 11.16. More precisely, consider a group $\Gamma$ with a finite presentation $\left\langle g_{1}, . ., g_{k} \mid R_{1}, . ., R_{m}\right\rangle$. Given $[\rho] \in$ $\mathcal{D}_{n-1}(\Gamma)$ define

$$
B_{n-1}([\rho]):=\inf _{x \in \mathbb{H}^{n}} \max _{i=1, \ldots, k} d\left(x, \rho\left(g_{i}\right)(x)\right) .
$$

Find an explicit constant $C$, which depends on $n, k, m$ and the lengths of the words $R_{i}$, so that the function $B_{n-1}: \mathcal{D}_{n-1}(\Gamma) \rightarrow \mathbb{R}$ is bounded from above by $C$.

Remark 11.19. In the case of Coxeter groups $\Gamma$, such explicit bound was obtained by Y. Lai [143]: The constant $C$ depends only on the rank of the Coxeter group and $n$.

Theorem 11.16 suggests that one should also look for geometric bounds on $[\rho] \in$ $\mathcal{D}_{n}(\Gamma)$ : Even if $\mathcal{D}_{n}(\Gamma)$ is noncompact (or its "size" is unknown), one can still try to find some natural functionals on $\mathcal{D}_{n}(\Gamma)$ and obtain explicit bounds (from below and from above) on these functionals.

Definition 11.20 (Diameter of a representation). Given a discrete embedding $\rho$ : $\Gamma \rightarrow \Gamma^{\prime}=\rho(\Gamma) \subset \operatorname{Mob}\left(\mathbb{S}^{n}\right)$, consider the set $S$ of connected subgraphs $\sigma \subset$ $\mathbb{H}^{n+1} / \Gamma^{\prime}$ with the property: The map $\pi_{1}(\sigma) \rightarrow \pi_{1}(M)$ is surjective.

Then the diameter of $\rho$ is

$$
\operatorname{diam}(\rho):=\inf \{\operatorname{length}(\sigma): \sigma \in S\} .
$$

Problem 11.21. Given a group $\Gamma$ as in Theorem 11.16, find explicit bounds on $\operatorname{diam}(\rho)$ (in terms of the presentation of $\Gamma$ ) for representations $[\rho] \in \mathcal{D}_{n}(\Gamma)$.

Note that the positive lower bound on $\operatorname{diam}(\rho)$ is an easy corollary of the Kazhdan-Margulis lemma.

Definition 11.22. (Volumes of a representation) Fix a homology class $[\zeta] \in Z_{p}(\Gamma)$, $2 \leq p \leq c d(\Gamma)$. For a representation $\rho \in \mathcal{D}_{n}(\Gamma)$ consider the quotient manifold $M=\mathbb{H}^{n} / \rho(\Gamma)$. Define the set $E(\zeta)$ of singular $p$-cycles $\zeta^{\prime} \in Z_{p}(M)$ which represent the homology class $[\zeta]$ under the isomorphism

$$
H_{p}(\Gamma) \rightarrow H_{p}(M)
$$

induced by the isomorphism $\rho: \Gamma \rightarrow \pi_{1}(M)$. Lastly, define the $\rho$-volume of the class $[\zeta]$ by

$$
\operatorname{Vol}_{\rho}(\zeta):=\inf \left\{\operatorname{Vol}\left(\zeta^{\prime}\right): \zeta^{\prime} \in E(\zeta)\right\}
$$

Let $\|\zeta\|$ denote the Gromov-norm of the class $[\zeta]$ and let $c_{p}$ denote the volume of the regular ideal geodesic $p$-simplex in $\mathbb{H}^{p}$. Then an easy application of Thurston's "chain-straightening" is the inequality

$$
\operatorname{Vol}_{\rho}(\zeta) \leq c_{p}\|\zeta\|
$$

for all $\rho,[\zeta]$ and $p \geq 2$. However good lower bounds on the volume are considerably more difficult to get. 
Given a hyperbolic manifold $M$ define $H_{p}^{p a r}(M)$ to be the image in $H_{p}(M)$ of the $p$-th homology group of the union of all cusps of $M$. Then for every parabolic class $[\zeta] \in H_{p}^{p a r}(M)$ and every $\rho$, we clearly have

$$
\operatorname{Vol}_{\rho}(\zeta)=0 .
$$

However there exists a positive constant $\epsilon=\epsilon(p, n)$ such that for every $p>0$, every non-cuspidal class $[\zeta]$ and every $\rho$, we obtain

$$
\operatorname{Vol}_{\rho}(\zeta) \geq \epsilon,
$$

see [121]. Below are some more interesting lower bounds on the volume:

Theorem 11.23. (Follows directly from $[86 \text {, Theorem } 5.38]^{9}$ ). Let $\Gamma$ be isomorphic to the fundamental group of a compact aspherical $k$-manifold $N$ and $[\zeta]=[N]$ be the fundamental class of $M$. Then there exists a universal (explicit) constant $c(p, n)>0$ depending only on $p$ and $n$, such that

$$
\operatorname{Vol}_{\rho}(\zeta) \geq c(p, n)\|N\| \text {. }
$$
10:

One gets better estimates using the work of Besson, Courtois and Gallot [21]

Theorem 11.24. Fix a representation $[\phi] \in \mathcal{D}_{n}(\Gamma)$. Then for every $[\rho] \in \mathcal{D}_{n}(\Gamma)$ and $p \geq 3$ we obtain

$$
\left(\frac{p}{n}\right)^{p+1} \operatorname{Vol}_{\phi}(\zeta) \leq \operatorname{Vol}_{\rho}(\zeta)
$$

For instance, if $\Gamma^{\prime}:=\phi(\Gamma)$ happens to be a uniform lattice in $\operatorname{Isom}\left(\mathbb{H}^{p}\right)$, we obtain

Corollary 11.25. For every $[\rho] \in \mathcal{D}_{n}(\Gamma)$ and $p \geq 3$ we have

$$
\left(\frac{p}{n}\right)^{p+1} \operatorname{Vol}\left(M^{\prime}\right) \leq \operatorname{Vol}_{\rho}(\zeta)
$$

where $M^{\prime}=\mathbb{H}^{p} / \Gamma^{\prime}$ and $[\zeta]$ is the fundamental class.

\subsection{Why is it so difficult to construct higher-dimensional geometrically infinite Kleinian groups?}

(i). The oldest trick for proving existence of geometrically infinite groups is due to L. Bers [19]:

Start with (say) a convex-cocompact subgroup $\Gamma \subset \operatorname{Mob}\left(\mathbb{S}^{n}\right)$. Let $Q(\Gamma) \subset$ $\mathcal{D}_{n}(\Gamma)$ be the (open) subset of representations induced by quasiconformal conjugation. Let $Q_{0}(\Gamma)$ denote the component of $Q(\Gamma)$ containing the (conjugacy class of) identity representation $\left[\rho_{0}\right]$. We assume that the closure of $Q_{0}(\Gamma)$ is not open in $X_{n}(\Gamma)$. Then there exists a curve $\left[\rho_{t}\right] \in X_{n}(\Gamma), t \in[0,1]$, so that $\rho_{1}$ is either nondiscrete or non-injective. Since $\mathcal{D}_{n}(\Gamma)$ is closed, it follows that there exists $s \in(0,1)$ such that $\left[\rho_{s}(\Gamma)\right]$ belongs to $\mathcal{D}_{n}(\Gamma)$ but $\rho_{s}(\Gamma)$ is not convex-cocompact.

${ }^{9} \mathrm{I}$ am grateful to A. Nabutovsky for this reference.

${ }^{10} \mathrm{I}$ am grateful to J. Souto for pointing this out. 
If $\Gamma^{\prime}=\rho_{s}(\Gamma)$ contains no parabolic elements, it would follow that $\Gamma^{\prime}$ is isomorphic to $\Gamma$ and is not geometrically finite. However, it could happen that the frontier of $Q_{0}(\Gamma)$ consists entirely of the classes $[\rho]$ for which $\rho(\Gamma)$ contains parabolic elements.

The latter cannot occur if $n=2$ for dimension reasons: The set of parabolic elements of $\operatorname{PSL}(2, \mathbb{C})$ has real codimension 2 and, hence, does not separate. However for all $n \neq 2$, the set of parabolic elements has real codimension 1 and this argument is inconclusive.

One can try to apply the above argument in the case of a codimension 1 fuchsian group $\Gamma \subset \operatorname{Mob}\left(\mathbb{S}^{n}\right)$ which acts as a cocompact lattice on $\mathbb{H}^{n} \subset \mathbb{H}^{n+1}$. Suppose that $M=\mathbb{H}^{n} / \Gamma$ contains a totally-geodesic compact hypersurface $S$. Then we have the circle $\mathbb{S}^{1}$ worth of bending deformations $\rho_{t}$ along $S$. As $t=\pi$, the image of $\rho_{t}$ is again contained in $\operatorname{Mob}\left(\mathbb{S}^{n-1}\right)$. Therefore $\rho_{\pi}$ is either nondiscrete or non-injective. However, conceivably, in all such cases, for $\left[\rho_{s}\right] \in \partial Q_{0}(\Gamma)$ the representation $\rho_{s}$ is geometrically finite (because its image may contain parabolic elements). It happens, for instance, if $\Gamma$ is a reflection group.

Note that even when $n=2$ and we are bending a 1 -fuchsian group $\Gamma$, it is hard to predict which simple closed geodesics $\alpha \subset \mathbb{H}^{2} / \Gamma$ yield geometrically infinite groups (via bending along $\alpha$ ).

(ii). One can try to construct explicit examples of fundamental domains, following, say, T. Jørgensen [104] or A. Marden and T. Jørgensen [105].

The trouble is that constructing fundamental polyhedra with infinitely many faces in $\mathbb{H}^{4}$ is quite a bit harder than in $\mathbb{H}^{3}$. One can try to find a lattice $\widehat{\Gamma} \subset$ $\operatorname{Mob}\left(\mathbb{S}^{3}\right)$ which contains a nontrivial finitely-generated normal subgroup $\Gamma$ of infinite index. ${ }^{11}$ This is, probably, the most promising approach, since it works for complex-hyperbolic lattices in $P U(2,1)$, cf. [115]. One can try to imitate Livne's examples, by constructing $\Gamma \subset \widehat{\Gamma}$ such that $\widehat{\Gamma} / \Gamma$ is isomorphic to a surface group. This would require coming up with a specific compact convex polyhedron in $\mathbb{H}^{4}$ such that the associated 4-manifold appears as a (singular) fibration over a surface.

(iii). One can try to use combinatorial group theory. Note that there are plenty of examples of (mostly 2-dimensional) Gromov-hyperbolic groups $\widehat{\Gamma}$ which contain nontrivial finitely-generated normal subgroups $\Gamma$ of infinite index. See, e.g., $[26,43,174,195]$ for the examples which are not 3-manifold groups. However embedding a given hyperbolic group $\widehat{\Gamma}$ in $\operatorname{Mob}\left(\mathbb{S}^{n}\right)$ is a nontrivial task, cf. [37, 118] and discussion in Section 12. The groups considered in [118], probably provide the best opportunity here, since most of them do not pass the perimeter test of J. McCammond and D. Wise [162]. (If a geometrically finite group $\widehat{\Gamma}$ satisfies the perimeter test, then every finitely-generated subgroup of $\widehat{\Gamma}$ is geometrically finite.)

(iv). What would geometrically infinite examples look like? Let $\Gamma \subset \operatorname{Mob}\left(\mathbb{S}^{2}\right)$ be a singly-degenerate group; assume for simplicity that the injectivity radius of

${ }^{11}$ If $\widehat{\Gamma}$ is a Kleinian group containing a nontrivial normal subgroup $\Gamma$ of infinite index, then $\Gamma$ is necessarily geometrically infinite. 
$\mathbb{H}^{3} / \Gamma$ is bounded away from zero. Let $S$ denote the boundary of

$$
\operatorname{Hull}(\Lambda(\Gamma)) / \Gamma
$$

and $\lambda \subset S$ be the ending lamination of $\Gamma$. Then every leaf of $\lambda$ lifts to an exponentially distorted curve $\kappa$ in $\mathbb{H}^{3}$ : Given points $x, y \in \kappa$, their extrinsic distance $d(x, y)$ in $\mathbb{H}^{3}$ is roughly the logarithm of their intrinsic distance along $\kappa$.

One would like to imitate this behavior in dimension 4 . Let $M$ be a closed hyperbolic 3-manifold containing an embedded compact totally-geodesic surface $S \subset M$. Let $\lambda \subset S$ be an ending lamination from the above example. One would like to construct a complete hyperbolic 4-manifold $N$ homotopy-equivalent to $M$, so that under the (smooth) homotopy-equivalence $f: M \rightarrow N$ we have:

For every leaf $L$ of $\lambda, f(L)$ lifts to an exponentially distorted curve in $\mathbb{H}^{4}$. Then $\pi_{1}(N)$ will necessarily be a geometrically infinite subgroup $\Gamma$ of $\operatorname{Mob}\left(\mathbb{S}^{4}\right)$. At the moment it is not even clear how to make this work with a hyperbolic metric on $N$ replaced by a complete Riemannian metric of negatively pinched sectional curvature, although constructing a Gromov-hyperbolic metric with this behavior is not that difficult. (Recall that a Riemannian metric is said to be negatively pinched if its sectional curvature varies between two negative numbers.) An example $\Gamma$ of this type is likely to have two components of $\Omega(\Gamma)$ : One contractible and one not.

More ambitiously, one can try to get a singly degenerate group $\Gamma \subset \operatorname{Mob}\left(\mathbb{S}^{3}\right)$ (so that $\Omega(\Gamma)$ is contractible and $M^{3}(\Gamma)$ is compact). How would such an example look like? One can imagine taking a 1-dimensional quasi-geodesic foliation $\lambda$ of the 3-manifold $M$ as above and then requiring that for every leaf $L \subset \lambda$, the curve $f(L)$ lifts to an exponentially distorted curve in $\mathbb{H}^{4}$. At the moment I do not see even a Gromov-hyperbolic model of this behavior. Another option would be to work with 2-dimensional laminations $\nu$ (with simply-connected leaves) in $M$ and require every leaf $L \subset \nu$ to correspond to an exponentially distorted surface in $\mathbb{H}^{4}$ (or a negatively-curved simply-connected 4-manifold), which limits to a single point in $\mathbb{S}^{3}$.

Problem 11.26. Construct a complete negatively pinched 4-dimensional Riemannian manifold $N$ homotopy-equivalent to a hyperbolic 3-manifold $M$, so that the convex core of $N$ either has exactly one boundary component or equals $N$ itself.

Question 11.27. Is there a geometrically infinite Kleinian subgroup of $\mathbf{M o b}\left(\mathbb{S}^{\mathrm{n}}\right)$ whose limit is homeomorphic to the Menger curve? Is there a geometrically infinite Kleinian subgroup of $\mathbf{M o b}\left(\mathbb{S}^{\mathrm{n}}\right)$ which is isomorphic to the fundamental group of a closed aspherical manifold of dimension $\geq 3$ ? Are there examples of such groups acting isometrically on complete negatively pinched manifolds? Are there examples of hyperbolic (or even negatively curved) 4-manifolds $M$ such that $\pi_{1}(M)=\Gamma$ fits into a short exact sequence

$$
1 \rightarrow \pi_{1}(S) \rightarrow \Gamma \rightarrow \pi_{1}(F) \rightarrow 1,
$$

where $S, F$ are closed hyperbolic surfaces? Note that there are no complex-hyperbolic examples of this type, see [115]. 


\section{Algebraic and topological constraints on Kleinian groups}

Sadly, there are only few known algebraic and topological restrictions on Kleinian subgroups in $\operatorname{Mob}\left(\mathbb{S}^{n}\right)$ that do not follow from the elementary restrictions, which come from the restrictions on geometry of complete negatively curved Riemannian manifolds. Examples of the elementary restrictions on a Kleinian group $\Gamma$ are:

1. Every solvable subgroup of a Kleinian group is virtually abelian.

2. The normalizer (in $\Gamma$ ) of an infinite cyclic subgroup of $\Gamma$ is virtually abelian.

3. Every elementary (i.e., virtually abelian) subgroup $\Delta \subset \Gamma$ is contained in a unique maximal elementary subgroup $\tilde{\Delta} \subset \Gamma$.

4. Every Kleinian group has finite (virtual) cohomological dimension.

In this section we review known nonelementary algebraic and topological restrictions on Kleinian groups.

\subsection{Algebraic constraints}

Definition 12.1. An abstract Kleinian group is a group $\Gamma$ which admits a discrete embedding in $\operatorname{Mob}\left(\mathbb{S}^{n}\right)$ for some $n$. Such a group is called elementary if it is virtually abelian.

In order to eliminate trivial restrictions on abstract Kleinian groups one can restrict attention to Gromov-hyperbolic Kleinian groups. Below is the list of known algebraic constraints on Kleinian groups under this extra assumption:

1. Kleinian groups are residually finite and virtually torsion-free. ${ }^{12}$ (This, of course, holds for all finitely generated matrix groups.)

2. Kleinian groups satisfy the Haagerup property, in particular, infinite Kleinian groups do not satisfy property $(\mathrm{T})$, see [55].

3. If a Kleinian group $\Gamma$ is Kähler, then $\Gamma$ is virtually isomorphic to the fundamental group of a compact Riemann surface. This is a deep theorem of J. Carlson and D. Toledo [52], who proved that every homomorphism of a Kähler group to $\mathbf{M o b}\left(\mathbb{S}^{n}\right)$ either factors through a virtually surface group, or its image fixes a point in $\mathbb{B}^{n+1}$.

Recall that a topological group $G$ is said to satisfy the Haagerup property if it admits a (metrically) proper continuous isometric action on a Hilbert space $H$. An action of a metrizable topological group $G$ on $H$ is metrically proper if for every bounded subset $B \subset H$, the set

$$
\{g \in G: g(B) \cap B \neq \emptyset\}
$$

is a bounded subset of $G$. Since $\operatorname{Mob}\left(\mathbb{S}^{n}\right)$ satisfies the Haagerup property for every $n$ (see, e.g., [55]), all Kleinian groups also do.

A group $\pi$ is called Kähler if it is isomorphic to the fundamental group of a compact Kähler manifold. For instance, every uniform lattice in $\mathbb{C H}^{n}$ is Kähler; therefore it cannot be an abstract Kleinian group unless $n=1$.

\footnotetext{
${ }^{12}$ It is widely believed that there are Gromov-hyperbolic groups which are not residually finite.
} 
Remark 12.2. A (finitely-generated) group satisfies the Haagerup property if and only if it admits an isometric (metrically) properly discontinuous action on the infinite dimensional hyperbolic space $\mathbb{H}^{\infty}$, see [85, 7.A.III]. The result of Carlson and Toledo shows that (for Gromov-hyperbolic groups) there are nontrivial obstructions to replacing these infinite-dimensional actions with finite-dimensional ones.

Observation 12.3. All currently known nontrivial restrictions on abstract Kleinian groups can be traced to 1,2 or 3 .

Problem 12.4. Find other restrictions on abstract Kleinian groups.

Potentially, some new restrictions would follow from the Rips-Thurston compactness theorem. The difficulty comes from the following. Let $\Gamma$ be a Gromovhyperbolic group which admits no nontrivial isometric actions on $\mathbb{R}$-trees. Then (see [116]) there exists $C<\infty$, such that for every sequence $\left[\rho_{j}\right] \in \mathcal{D}_{n}(\Gamma)$, we obtain a uniform bound

$$
B_{n}\left(\left[\rho_{j}\right]\right) \leq C,
$$

where $B_{n}: \mathcal{D}_{n}(\Gamma) \rightarrow \mathbb{R}$ is the minimax function defined in Problem 11.18. If $n$ were fixed, then the sequence $\left(\rho_{j}\right)$ would subconverge to a representation to $\mathbf{M o b}\left(\mathbb{S}^{\mathrm{n}}\right)$ (for some choice of representations $\rho_{j}$ in the classes $\left[\rho_{j}\right]$ ). However, since we are not fixing the dimension of the hyperbolic space on which our $\Gamma$ is supposed to act, the inequality (12.1) does not seem to yield any useful information. By taking an ultralimit of $\rho_{j}$ 's we will get an action of $\Gamma$ on an infinite-dimensional hyperbolic space. This action, however, may have a fixed point, since

$$
\lim _{n \rightarrow \infty} \mu_{n}=0
$$

where $\mu_{n}$ is the Margulis constant for $\mathbb{H}^{n+1}$. See also Remark 12.2.

Example. Let $M^{3}$ be a closed non-Haken hyperbolic 3-manifold, so that $\Pi:=$ $\pi_{1}(M)$ contains a maximal 1 -fuchsian subgroup $F$. For each automorphism $\phi$ : $F \rightarrow F$ we define the HNN extension

$$
\Gamma_{\phi}:=\Pi *_{F} \cong_{\phi} F=\left\langle\Pi, t \mid t g t^{-1}=\phi(g), \forall g \in F\right\rangle .
$$

Then $\Gamma_{\phi}$ is Gromov-hyperbolic for all pseudo-Anosov automorphisms $\phi$, see [27]. It is a direct corollary of Theorem 11.16 that for every $n$, only finitely many of the groups $\Gamma_{\phi}$ embed in $\mathbf{M o b}\left(\mathbb{S}^{n}\right)$ as Kleinian subgroups. Is it true that there exists a pseudo-Anosov automorphism $\phi$ such that $\Gamma_{\phi}$ is not an abstract Kleinian group?

Infinite finitely-generated Gromov-hyperbolic Coxeter groups are all linear, satisfy the Haagerup property and are not Kähler (except for the virtually surface groups).

Problem 12.5. Is it true that every finitely-generated Gromov-hyperbolic Coxeter group $\Gamma$ is an abstract Kleinian group? 
Note that there are Gromov-hyperbolic Coxeter groups $\Gamma$ which do not admit discrete embeddings $\rho: \Gamma \rightarrow \operatorname{Mob}\left(\mathbb{S}^{n}\right)$ (for any $n$ ), so that the Coxeter generators of $\Gamma$ act as reflections in the faces of a fundamental domain of $\rho(\Gamma)$, see [76].

The answer to the next question is probably negative, but the examples would be tricky to construct:

Question 12.6. Is it true that a group weakly commensurable to a Kleinian group is also a Kleinian group? Even more ambitiously: Is the property of being Kleinian a quasi-isometry invariant of a group?

Recall that two groups $\Gamma$ and $\Gamma^{\prime}$ are called weakly commensurable if there exists a chain of groups and homomorphisms

$$
\Gamma=\Gamma_{0} \rightarrow \Gamma_{1} \leftarrow \Gamma_{2} \rightarrow \Gamma_{3} \cdots \leftarrow \Gamma_{k-1} \rightarrow \Gamma_{k}=\Gamma^{\prime},
$$

where each arrow $\Gamma_{i} \rightarrow \Gamma_{i \pm 1}$ is a homomorphism whose kernel and cokernel are finite.

There are few more known algebraic restrictions on geometrically finite Kleinian groups. All such groups are relatively hyperbolic.

We recall that a group $\Gamma$ is called cohopfian if every injective endomorphism $\Gamma \rightarrow \Gamma$ is also surjective.

Remark 12.7. A group $\Gamma$ is called hopfian if every epimorphism $\Gamma \rightarrow \Gamma$ is injective. Every residually finite group $\Gamma$ is hopfian, see [151]. In particular, every Kleinian group is hopfian.

For instance, free groups and free abelian groups are not cohopfian. More generally, if $\Gamma$ splits as a nontrivial free product,

$$
\Gamma \cong \Gamma_{1} * \Gamma_{2},
$$

then $\Gamma$ is not cohopfian: Indeed, for nontrivial elements $\gamma_{1} \in \Gamma_{1}, \gamma_{2} \in \Gamma_{2}$, set $\alpha:=\gamma_{1} \gamma_{2}$, and

$$
\Gamma_{1}^{\prime}:=\alpha \Gamma_{1} \alpha^{-1}
$$

Then

$$
\Gamma \cong \Gamma_{1}^{\prime} * \Gamma_{2}
$$

is a proper subgroup of $\Gamma$. On the other hand, lattices in $\operatorname{Isom}\left(\mathbb{H}^{n}\right), n \geq 3$, and uniform lattices in $\operatorname{Isom}\left(\mathbb{H}^{2}\right)$ are cohopfian. Indeed, Mostow rigidity theorem implies that if $M_{1}, M_{2}$ are hyperbolic $n$-manifolds of finite volume (and $n \geq 3$ ) or compact hyperbolic surfaces, and $M_{1} \rightarrow M_{2}$ is a $d$-fold covering, then

$$
\operatorname{Vol}\left(M_{1}\right)=d \operatorname{Vol}\left(M_{2}\right) \text {. }
$$

On the other hand, if $M$ is a hyperbolic manifold of finite volume (or a compact hyperbolic surfaces), then every proper embedding

$$
\pi_{1}(M) \rightarrow \pi_{1}(M)
$$

induces a $d$-fold covering $M \rightarrow M$, with $d \in\{2,3, \ldots, \infty\}$. Hence $\pi_{1}(M)$ is cohopfian. 
If a Kleinian group $\Gamma \subset \operatorname{Mob}\left(\mathbb{S}^{n}\right)$ fails to be cohopfian, we can iterate a proper embedding $\phi: \Gamma \rightarrow \Gamma$, thereby obtaining a sequence of discrete and faithful representations

$$
\rho_{i}=\underbrace{\phi \circ \cdots \circ \phi}_{i \text { times }}
$$

of $\Gamma$ into $\operatorname{Mob}\left(\mathbb{S}^{n}\right)$. By analyzing such sequences, T. Delzant and L. Potyagailo [63] obtained a characterization of geometrically finite Kleinian groups which are cohopfian. We will need two definitions in order to describe their result.

Definition 12.8. If $\Gamma$ is a Kleinian group and $\Delta \subset \Gamma$ is an elementary subgroup, let $\tilde{\Delta}$ denote the maximal elementary subgroup of $\Gamma$ containing $\Delta$.

Definition 12.9. Suppose a group $\Gamma$ splits as a graph of groups

$$
\Gamma \cong \pi_{1}\left(\mathcal{G}, \Gamma_{e}, \Gamma_{v}\right),
$$

and suppose that edge groups $\Gamma_{e}$ of this graph are elementary. We say that the edge group $\Gamma_{e}$ is essentially non-maximal if the subgroup $\tilde{\Gamma}_{e} \subset \Gamma$, is not conjugate into any of the vertex subgroups of the graph of groups $\mathcal{G}$. The splitting is essentially non-maximal if there exists at least one such an edge group. Otherwise we say that the splitting is essentially maximal.

For instance, if every edge subgroup is a maximal elementary subgroup of $\Gamma$, then the splitting is essentially maximal.

Theorem 12.10. (T. Delzant and L. Potyagailo [63].) Let $\Gamma$ be a non-elementary, geometrically finite, one-ended Kleinian group without 2-torsion. Then $\Gamma$ is cohopfian if and only if the following two conditions are satisfied:

1) $\Gamma$ has no essentially non-maximal splittings.

2) $\Gamma$ does not split as an amalgamated free product

$$
\Gamma=\Gamma_{1} * \Gamma_{3} \tilde{\Gamma}_{3},
$$

with $\tilde{\Gamma}_{3}$ maximal elementary, such that the normal closure of the subgroup $\Gamma_{3}$ in $\tilde{\Gamma}_{3}$ is of infinite index in $\tilde{\Gamma}_{3}$.

One of the ingredients in the proof of this theorem was the fact that nonelementary geometrically finite groups $\Gamma$ do not contain subgroups $\Gamma^{\prime}$, which are conjugate to $\Gamma$ in $\operatorname{Mob}\left(\mathbb{S}^{n}\right)$, see [223].

Question 12.11 (L. Potyagailo). Let $\Gamma \subset \operatorname{Mob}\left(\mathbb{S}^{n}\right)$ be a finitely generated nonelementary Kleinian group. Suppose that $\alpha \in \mathbf{M o b}\left(\mathbb{S}^{n}\right)$ is such that

$$
\Gamma^{\prime}=\alpha \Gamma \alpha^{-1} \subset \Gamma \text {. }
$$

Does it follow that $\Gamma^{\prime}=\Gamma$ ?

The affirmative answer to this question for $n=2$ was given in a paper of L. Potyagailo and K.-I. Ohshika [178] (modulo Tameness Conjecture, Theorem $10.3)$. 
Question 12.12. Is the isomorphism problem solvable within the class of all finitelypresented Kleinian groups? Note that the work of F. Dahmani and D. Groves [59] implies solvability of the isomorphism problem in the category of geometrically finite Kleinian groups.

It was proved by M. Bonk and O. Schramm [36] that every Gromov-hyperbolic group $\Gamma$ embeds quasi-isometrically in the usual hyperbolic space $\mathbb{H}^{n}$ for some $n=n(\Gamma)$. A natural question is if one can prove an equivariant version of this result. Note that there are many Gromov-hyperbolic groups which are not Kleinian, e.g., groups with property (T) and Gromov-hyperbolic Kähler groups. Therefore one has to relax the isometric assumption. The natural category for this is the uniformly quasiconformal actions. Such an action is a monomorphism

$$
\rho: \Gamma \hookrightarrow Q C\left(\mathbb{S}^{n}\right)
$$

whose image consists of $K$-quasiconformal homeomorphisms with $K$ depending only on $\rho$.

Problem 12.13. Let $\Gamma$ be a Gromov-hyperbolic group. Does $\Gamma$ admit a uniformly quasiconformal discrete action on $\mathbb{S}^{n}$ for some $n$ ? For instance, is there such an action if $\Gamma$ is a uniform lattice in $P U(n, 1)$ or satisfies the property $(\mathrm{T})$ ?

T. Farrell and J. Lafont [74] proved that the topological counterpart of this problem has positive solution. A corollary of their work is that every Gromovhyperbolic group $\Gamma$ admits a convergence action $\rho$ on the closed $n$-ball, so that the limit set of $\Gamma^{\prime}=\rho(\Gamma)$ is equivariantly homeomorphic to the ideal boundary of $\Gamma$ and $\Omega\left(\Gamma^{\prime}\right) / \Gamma^{\prime}$ is compact and connected. We refer the reader to [78] for the definition of a convergence action.

\subsection{Topological constraints}

The basic problem here is to find topological restrictions on the hyperbolic manifold $\mathbb{H}^{n+1} / \Gamma$ and on the conformally-flat manifold $\Omega(\Gamma) / \Gamma$, which do not follow from the algebraic restrictions on the group $\Gamma$ and from the general algebraic topology restrictions (e.g., vanishing of the characteristic classes). There are only few nontrivial results in this direction. For $n=3$ we have:

Theorem 12.14. (M. Kapovich [109].) There exists a function $c(\chi)$ with the following property. Let $S$ be a closed hyperbolic surface. Suppose that $M^{4}$ is a complete hyperbolic 4-manifold which is homeomorphic to the total space of an $\mathbb{R}^{2}$-bundle $\xi: E \rightarrow S$ with the Euler number $e(\xi)$. Then

$$
|e(\xi)| \leq c(\chi(S))
$$

More generally,

Theorem 12.15. (M. Kapovich [109].) There exists a function $C\left(\chi_{1}, \chi_{2}\right)$ with the following property. Suppose that $M^{4}$ is a complete oriented hyperbolic 4-manifold. Let $\sigma_{j}: \Sigma_{j} \rightarrow M^{4}(j=1,2)$ be $\pi_{1}$-injective maps of closed oriented surfaces $\Sigma_{j}$. Then

$$
\left|\left\langle\sigma_{1}, \sigma_{2}\right\rangle\right| \leq C\left(\chi\left(\Sigma_{1}\right), \chi\left(\Sigma_{2}\right)\right)
$$


Here $\langle$,$\rangle is the intersection pairing on H_{2}\left(M^{4}\right)$. The bounds appearing in these theorems are explicit but astronomically high. The expected bounds are linear in $\chi(S)$ and $\chi\left(S_{i}\right), i=1,2$, cf. [87].

Other known restrictions are applications of the compactness theorem 11.16 and therefore explicit bounds in the following theorems are unknown.

Theorem 12.16. (M. Kapovich [111].) Given a closed hyperbolic n-manifold $B(n \geq$ 3 ) there exists a number $c(B)$ so that the following is true. Suppose that $M^{2 n} a$ complete hyperbolic $2 n$-manifold which is homeomorphic to the total space of an $\mathbb{R}^{n}$-bundle $\xi: E \rightarrow B$ with the Euler number $e(\xi)$. Then

$$
|e(\xi)| \leq c(B) .
$$

I. Belegradek greatly improved this result:

Theorem 12.17. (I. Belegradek [16].) Given a closed hyperbolic n-manifold $B(n \geq$ $3)$ there exists a number $C(B, k)$ so that the number of inequivalent $\mathbb{R}^{k}$-bundles $\xi: E \rightarrow B$ whose total space admits a complete hyperbolic metric, is at most $C(B, k)$.

Given a group $\pi$, let $\mathcal{M}_{\pi, n}$ denote the set of $n$-manifolds, whose fundamental group is isomorphic to $\pi$ and which admit complete hyperbolic metrics.

Theorem 12.18. (I. Belegradek [17].) Suppose that $\pi$ is a finitely-presented group with finite Betti numbers. Assume that $\pi$ does not split as an amalgam over a virtually abelian subgroup. The set $\mathcal{M}_{\pi, n}$ breaks into finitely many intersection preserving homotopy types.

\section{References}

[1] W. AвікоғF, Kleinian groups - geometrically finite and geometrically perverse, in "Geometry of group representations", vol. 74 of Contemp. Math., Amer. Math. Soc., 1988, pp. 1-50.

[2] I. AGol, Tameness of hyperbolic 3-manifolds. Preprint math.GT/0405568, 2004.

[3] L. Ahlfors, Finitely generated Kleinian groups, Amer. J. of Math., 86 (1964), pp. 413-429.

[4] - Remarks on the limit point set of a finitely generated Kleinian group., in "Advances in the Theory of Riemann Surfaces" (Proc. Conf., Stony Brook, 1969), Ann. of Math. Studies, No. 66, Princeton Univ. Press, Princeton, N.J., 1971, pp. 19 26.

[5] _ Ergodic properties of Moebius transformations, in "Analytic functions", vol. 798 of Springer Lecture Notes in Mathematics, Springer, 1980, pp. 19-25.

[6] — Möbius transformations in several dimensions, Ordway Professorship Lectures in Mathematics, University of Minnesota School of Mathematics, Minneapolis, Minn., 1981. 
[7] S. Akbulut And J. MCCARthy, Casson's invariant for oriented homology 3spheres, an exposition, Mathematical Notes 36, Princeton University Press, Princeton, 1990.

[8] P. S. Alexandroff, Einführung in die Mengenlehre und in die allgemeine Topologie, Hochschulbücher für Mathematik, 85, 1984.

[9] B. Apanasov, Bending and stamping deformations of hyperbolic manifolds, Ann. Global Anal. Geom., 8 (1990), pp. 3-12.

[10] —, Discrete groups in space and uniformization problems, Kluwer Academic Publishers Group, Dordrecht, 1991.

[11] B. Apanasov and A. Tetenov, Nontrivial cobordisms with geometrically finite hyperbolic structures, J. Differential Geom., 28 (1988), pp. 407-422.

[12] —, Deformations of hyperbolic structures along surfaces with boundary and pleated surfaces, in "Low-dimensional topology" (Knoxville, 1992), Internat. Press, 1994, pp. 1-14.

[13] A. Bart and K. Scannell, The generalized cuspidal cohomology problem, Canad. J. Math., 58 (2006), pp. 673-690.

[14] A. BeARDON AND B. MASKiT, Limit points of Kleinian groups and finite sided fundamental polyhedra, Acta Math., 132 (1974), pp. 1-12.

[15] I. BelegradeK, Some curious Kleinian groups and hyperbolic 5-manifolds, Transform. Groups, 2 (1997), pp. 3-29.

[16] _ Intersections in hyperbolic manifolds, Geometry and Topology, 2 (1998), pp. 117-144.

[17] - Pinching, Pontrjagin classes, and negatively curved vector bundles, Invent. Math., 144 (2001), pp. 353-379.

[18] R. Benedetti and C. Pertonio, Lectures on Hyperbolic Geometry, Springer, 1992.

[19] L. BERs, On the boundaries of Teichmüller spaces and on Kleinian groups, I, Annals of Math., 91 (1970), pp. 570-600.

[20] _ Uniformization, moduli and Kleinian groups, Bull. London Math. Society, 4 (1972), pp. 257-300.

[21] G. Besson, G. Courtois, And S. Gallot, Lemme de Schwarz réel et applications géométriques, Acta Math., 183 (1999), pp. 145-169.

[22] — Hyperbolic manifolds, amalgamated products and critical exponents, C. R. Math. Acad. Sci. Paris, 336 (2003), pp. 257-261.

[23] M. Bestvina, Degenerations of hyperbolic space, Duke Math. Journal, 56 (1988), pp. $143-161$

[24] M. Bestvina AND N. BRADy, Morse theory and finiteness properties of groups, Inventiones Math., 129 (1997), pp. 445-470.

[25] M. Bestvina And D. Cooper, A wild Cantor set as the limit set of a conformal group action on $\mathbf{S}^{3}$, Proc. of AMS, 99 (1987), pp. 623-627.

[26] M. Bestvina And M. Feighn, A combination theorem for negatively curved groups, J. Differential Geom., 35 (1992), pp. 85-101.

[27] - Stable actions of groups on real trees, Invent. Math., 121 (1995), pp. 287-321. 
[28] M. Bestrina And G. Mess, The boundary of negatively curved groups, Journal of the AMS, 4 (1991), pp. 469-481.

[29] R. BIERI, Homological dimension of discrete groups, Mathematical Notes, Queen Mary College, 1976.

[30] R. Bieri and B. Eckmann, Relative homology and Poincaré duality for group pairs, J. Pure Appl. Algebra, 13 (1978), pp. 277-319.

[31] R.H. Bing, The geometric topology of 3-manifolds, vol. 40 of American Mathematical Society Colloquium Publications, American Mathematical Society, Providence, RI, 1983.

[32] C. Bishop, On a theorem of Beardon and Maskit, Ann. Acad. Sci. Fenn. Math., 21 (1996), pp. 383-388.

[33] C. Bishop And P. Jones, Hausdorff dimension and Kleinian groups, Acta Math., 179 (1997), pp. 1-39.

[34] F. Bonahon, Boutes des varietes hyperboliques de dimension trois, Ann. of Math, 124 (1986), pp. 71-158.

[35] M. Bonk AND B. KLeIneR, Rigidity for quasi-Fuchsian actions on negatively curved spaces, Int. Math. Res. Not., (2004), pp. 3309-3316.

[36] M. Bonk And O. Schramm, Embeddings of Gromov hyperbolic spaces, Geom. Funct. Anal., 10 (2000), pp. 266-306.

[37] M. Bourdon, Sur la dimension de Hausdorff de l'ensemble limite d'une famille de sous-groupes convexes co-compacts, C. R. Acad. Sci. Paris Sér. I Math., 325 (1997), pp. 1097-1100.

[38] M. Bourdon, F. MARTin, AND A. VAlEtte, Vanishing and non-vanishing for the first $L^{p}$-cohomology of groups, Comment. Math. Helv., 80 (2005), pp. 377-389.

[39] B.H. BowDitch, Geometrical finiteness for hyperbolic groups, Journal of Functional Anal., 113 (1993), pp. 245-317.

[40] B.H. Bowditch, Spaces of geometrically finite representations, Ann. Acad. Sci. Fenn. Math., 23 (1998), pp. 389-414.

[41] B.H. Bowditch, Planar groups and the Seifert conjecture, J. Reine Angew. Math., 576 (2004), pp. 11-62.

[42] B.H. Bowditch And G. Mess, A 4-dimensional Kleinian group, Transactions of AMS, 14 (1994), pp. 391-405.

[43] N. BRADY, Branched coverings of cubical complexes and subgroups of hyperbolic groups, J. London Math. Soc. (2), 60 (1999), pp. 461-480.

[44] M. Bridson And A. HAefliger, Metric spaces of non-positive curvature, SpringerVerlag, Berlin, 1999.

[45] M. BRIN, Torsion-free action on 1-acyclic manifolds and the loop theorem, Topology, 20 (1981), pp. 353-364.

[46] J. Brock, R. Canary, and Y. Minsky, The classification of Kleinian surface groups, II: The Ending Lamination Conjecture. Preprint, 2004.

[47] K. Brown, Cohomology of Groups, vol. 87 of Graduate Texts in Mathematics, Springer, 1982. 
[48] E. CAlaBi, On compact Riemannian manifolds with constant curvature, in Proc. Sympos. Pure Math., Vol. III, American Mathematical Society, Providence, R.I., 1961, pp. 155-180.

[49] D. Calegari and D. Gabai, Shrinkwrapping and the taming of hyperbolic 3manifolds, J. Amer. Math. Soc., 19 (2006), pp. 385-446.

[50] R. CAnary and E. TAYlor, Hausdorff dimension and limits of Kleinian groups, Geom. Funct. Anal., 9 (1999), pp. 283-297.

[51] J. Cannon and W.P. Thurston, Group invariant Peano curves. Preprint.

[52] J. Carlson And D. Toledo, Harmonic mappings of Kähler manifolds to locally symmetric spaces, Inst. Hautes Études Sci. Publ. Math., (1989), pp. 173-201.

[53] J. Casson AND S. Bleiler, Automorphisms of Surfaces After Nielsen and Thurston, vol. 9 of LMS Student Texts, Cambridge University Press, 1988.

[54] A. Chang, J. QING, AND P. YANG, On finiteness of Kleinian groups in general dimension, J. Reine Angew. Math., 571 (2004), pp. 1-17.

[55] P.-A. Cherix, M. Cowling, P. Jolissaint, P. Julg, and A. Valette, Groups with the Haagerup property, vol. 197 of Progress in Mathematics, Birkhäuser, 2001.

[56] V. Chuckrow, Schottky groups with applications to Kleinian groups, Ann. of Math., 88 (1968), pp. 47-61.

[57] S. Claytor, Topological immersion of Peanian continua in the spherical surface, Ann. of Math., 35 (1924), pp. 809-835.

[58] D. Cooper, D. Long, And A. Reid, Bundles and finite foliations, Inventiones Mathematicae, 118 (1994), pp. 255-283.

[59] F. Dahmani And D. Groves, The isomorphism problem for toral relatively hyperbolic groups. Preprint, 2005.

[60] M.W. DAvis, A hyperbolic 4-manifold, Proc. Amer. Math. Soc., 93 (1985), pp. 325328.

[61] D.G. DeGryse And R.P. Osborne, A wild Cantor set in $E^{n}$ with simply connected complement, Fund. Math., 86 (1974), pp. 9-27.

[62] P. Deligne And G.D. Mostow, Commensurabilities among lattices in $\mathrm{PU}(1, n)$, vol. 132 of Annals of Mathematics Studies, Princeton University Press, 1993.

[63] T. Delzant and L. Potyagailo, Endomorphisms of Kleinian groups, Geom. Funct. Anal., 13 (2003), pp. 396-436.

[64] S. Donaldson And D. Sullivan, Quasiconformal 4-manifolds, Acta Math., (1989), pp. 181-252.

[65] A. DouAdy AND C. EARle, Conformally natural extension of homeomorphisms of the circle, Acta Mathematica, 157 (1986), pp. 23-48.

[66] M.J. Dunwoody, Accessibility and groups of cohomological dimension one, Proc. London Math. Soc. (3), 38 (1979), pp. 193-215.

[67] B. Eckmann And P. Linnel, Poincaré dualtiy groups of dimension 2, II, Comm. Math. Helv., 58 (1983), pp. 111-114.

[68] B. Eckmann and H. Müller, Poincaré duality groups of dimension two, Comm. Math. Helv., 55 (1980), pp. 510-520. 
[69] D. Epstein, R. Canary, and P. Green, Notes on notes of Thurston, in "Analytical and geometric aspects of hyperbolic space", vol. 111 of London Math. Soc. Lecture Notes, Cambridge Univ. Press, 1987, pp. 3-92.

[70] F. FARrell And L. Jones, Negatively curved manifolds with exotic smooth structures, J. Amer. Math. Soc., 2 (1989), pp. 899-908.

[71] _ A topological analogue of Mostow's rigidity theorem, Journal of the AMS, 2 (1989), pp. 257-370.

[72] — Topological rigidity for compact non-positively curved manifolds, in "Differential geometry"., vol. 54 of Proc. of Symp. in Pure Math., 1993, pp. 229-274.

[73] — Rigidity for aspherical manifolds with $\pi_{1} \subset G L_{m}(R)$, Asian J. Math., 2 (1998), pp. 215-262.

[74] F.T. FARRELL AND J.-F. LAFONT, EZ-structures and topological applications, Comment. Math. Helv., 80 (2005), pp. 103-121.

[75] M. Feighn And G. Mess, Conjugacy classes of finite subgroups in Kleinian groups, Amer. J. of Math., 113 (1991), pp. 179-188.

[76] A. Felikson And P. Tumarkin, A series of word-hyperbolic Coxeter groups. Preprint, 2005.

[77] D. Garity, D. Repovš, And M. Želuko, Rigid Cantor sets in $\mathbf{R}^{3}$ with simply connected complement, Proc. Amer. Math. Soc., 134 (2006), pp. 2447-2456.

[78] F.W. Gehring And G.J. Martin, Discrete convergence groups, in "Complex analysis, I" (College Park, Md., 1985-86), Springer, Berlin, 1987, pp. 158-167.

[79] W. Goldman, Geometric structures on manifolds and varieties of representations, in "Geometry of group representations", vol. 74 of Contemporary Mathematics, 1987, pp. 169-198.

[80] _ Complex hyperbolic Kleinian groups, in "Complex Geometry". Proceedings of the Osaka International Conference, vol. 143 of Lecture notes in pure and applied mathematics, 1992, pp. 31-52.

[81] — Complex hyperbolic geometry, Oxford Mathematical Monographs, Oxford University Press, 1999.

[82] W. Goldman and J. J. Millson, The deformation theory of representations of fundamental groups of compact Kähler manifolds, Math. Publications of IHES, 67 (1988), pp. 43-96.

[83] L. Greenberg, On a theorem of Ahlfors and conjugate subgroups of Kleinian groups, Amer. J. of Math., 89 (1967), pp. 56-68.

[84] - Homomorphisms of triangle groups into PSL $(2, \mathbf{C})$, in Riemann surfaces and related topics: Proceedings of the 1978 Stony Brook Conference, vol. 97 of Ann. of Math. Stud., Princeton Univ. Press, 1981, pp. 167-181.

[85] M. Gromov, Asymptotic invariants of infinite groups, in "Geometric groups theory", volume 2, Proc. of the Symp. in Sussex 1991, G. Niblo and M. Roller, eds., vol. 182 of Lecture Notes series, Cambridge University Press, 1993.

[86] — Metric structures for Riemannian and non-Riemannian spaces, Birkhäuser Boston, 1999.

[87] M. Gromov, H. Lawson, and W. Thurston, Hyperbolic 4-manifolds and conformally flat 3-manifolds, Publ. Math. of IHES, 68 (1988), pp. 27-45. 
[88] M. Gromov and I. Piatetski-Shapiro, Non-arithmetic groups in Lobachevsky spaces, Publ. Math. of IHES, 66 (1988), pp. 93-103.

[89] M. Gromov and W. Thurston, Pinching constants for hyperbolic manifolds, Inventiones Mathematicae, 89 (1987), pp. 1-12.

[90] N. Gusevskĭ, Geometric decomposition of spatial Kleinian groups, Soviet Math. Dokl., 38 (1989), pp. 98-101.

[91] — Strange actions of free Kleinian groups, Sibirsk. Mat. Zh., 37 (1996), pp. 90107.

[92] N. Gusevskil AND N. Zindinova, Characterization of extended Schottky groups, Soviet Math. Doklady, 33 (1986), pp. 239-241.

[93] E. Hamilton, Geometric finiteness for hyperbolic orbifolds, Topology, 37 (1998), pp. $635-657$

[94] J. Heinonen, Lectures on analysis on metric spaces, Universitext, Springer-Verlag, New York, 2001.

[95] J. Hempel, 3-manifolds, vol. 86 of Annals of Mathematics Studies, Princeton University Press, 1976.

[96] W. Hurewicz and H. Wallman, Dimension Theory, Princeton University Press, 1941.

[97] S. HwAng, Moebius structures on 3-manifolds. Ph. D. Thesis, University of Utah, 2001.

[98] D. Ivanšić, J.G. Ratcliffe, And S.T. Tschantz, Complements of tori and Klein bottles in the 4-sphere that have hyperbolic structure, Algebr. Geom. Topol., 5 (2005), pp. 999-1026.

[99] T. IwANIEC AND G. MARTIN, Geometric function theory and non-linear analysis, Oxford Mathematical Monographs, Oxford University Press, New York, 2001.

[100] H. IZEKI, Limit sets of Kleinian groups and conformally flat Riemannian manifolds, Invent. Math., 122 (1995), pp. 603-625.

[101] — Quasiconformal stability of Kleinian groups and an embedding of a space of flat conformal structures, Conform. Geom. Dyn., 4 (2000), pp. 108-119.

[102] - Convex-cocompactness of Kleinian groups and conformally flat manifolds with positive scalar curvature, Proc. Amer. Math. Soc., 130 (2002), pp. 3731-3740.

[103] D. Johnson And J.J. Millson, Deformation spaces associated to compact hyperbolic manifolds, in "Discrete Groups in Geometry and Analysis", Papers in honor of G.D. Mostow on his 60th birthsday, vol. 67 of Progress in Mathematics, Birkhäuser, 1987, pp. 48-106.

[104] T. JøRGEnsen, Compact 3-manifolds of constant negative curvature fibering over the circle, Ann. of Math., 106 (1977), pp. 61-72.

[105] T. Jørgensen And A. Marden, Two doubly degenerate groups, Quart. J. Math. Oxford Ser. (2), 30 (1979), pp. 143-156.

[106] - Generic fundamental polyhedra for Kleinian groups, in "Holomorphic functions and moduli", Vol. II (Berkeley, 1986), vol. 11 of Math. Sci. Res. Inst. Publ., Springer, New York, 1988, pp. 69-85.

[107] M. Kapovich, Flat conformal structures on three-dimensional manifolds: the existence problem. I, Sibirsk. Mat. Zh., 30 (1989), pp. 60-73. 
[108] - Deformation spaces of flat conformal structures, in "Proceedings of the Second Soviet-Japan Symposium of Topology" (Khabarovsk, 1989). Questions and Answers in General Topology, 8 (1990), pp. 253-264.

[109] _ Intersection pairing on hyperbolic 4-manifolds. Preprint, MSRI, 1992.

[110] - Topological aspects of Kleinian groups in several dimensions. Preprint, MSRI, 1992.

[111] - Flat conformal structures on 3-manifolds. I. Uniformization of closed Seifert manifolds, J. Differential Geom., 38 (1993), pp. 191-215.

[112] — Hyperbolic 4-manifolds fibering over surfaces. Preprint, 1993.

[113] — Deformations of representations of discrete subgroups of $S O(3,1)$, Math. Ann., 299 (1994), pp. 341-354.

[114] _ On the absence of Sullivan's cusp finiteness theorem in higher dimensions, in "Algebra and analysis" (Irkutsk, 1989), Amer. Math. Soc., Providence, RI, 1995, pp. $77-89$.

[115] — On normal subgroups in the fundamental groups of complex surfaces. Preprint, 1998.

[116] - Hyperbolic manifolds and discrete groups, Birkhäuser Boston Inc., Boston, MA, 2001.

[117] — Conformally flat metrics on 4-manifolds, J. Differential Geom., 66 (2004), pp. 289-301.

[118] —, Representations of polygons of finite groups, Geom. Topol., 9 (2005), pp. 1915-1951.

[119] - Convex projective Gromov-Thurston examples. Preprint, 2006.

[120] - Lectures on the geometric group theory. Preprint, 2006.

[121] - Homological dimension and critical exponent of Kleinian groups. Preprint, 2007.

[122] M. Kapovich And B. Kleiner, Hyperbolic groups with low-dimensional boundary, Ann. Sci. École Norm. Sup. (4), 33 (2000), pp. 647-669.

[123] - Geometry of quasi-planes. Preprint, 2004.

[124] Coarse Alexander duality and duality groups, Journal of Diff. Geometry, 69 (2005), pp. 279-352.

[125] M. Kapovich And J. J. Millson, Bending deformations of representations of fundamental groups of complexes of groups. Preprint, 1996.

[126] - On the deformation theory of representations of fundamental groups of closed hyperbolic 3-manifolds, Topology, 35 (1996), pp. 1085-1106.

[127] M. Kapovich And L. Potyagailo, On absence of Ahlfors' and Sullivan's finiteness theorems for Kleinian groups in higher dimensions, Siberian Math. Journ., 32 (1991), pp. 227-237.

[128] - On absence of Ahlfors' finiteness theorem for Kleinian groups in dimension 3, Topology and its Applications, 40 (1991), pp. 83-91.

[129] M. Kapovich, L. Potyagailo, and E.B. Vinberg, Non-coherence of some nonuniform lattices in $\operatorname{Isom}\left(\mathbb{H}^{n}\right)$, Geometry and Topology, (to appear).

[130] D. Kazhdan and G. Margulis, A proof of Selberg's hypothesis, Math. Sbornik, 75 (1968), pp. 162-168. 
[131] R. KIRBy, Problems in low-dimensional topology, in "Geometric topology" (Athens, GA, 1993), Providence, RI, 1997, Amer. Math. Soc., pp. 35-473.

[132] R. Kirby and L. Siebenmann, Foundational essays on topological manifolds, smoothings and triangulations, vol. 88 of Annals of Mathematics Studies, Princeton University Press, 1977.

[133] B. KLEINER. In preparation.

[134] B. Kleiner And J. Lott, Notes of Perelman's papers. Preprint, math.DG/0605667, 2006.

[135] C. Kourouniotis, Deformations of hyperbolic structures, Math. Proc. Cambridge Philos. Soc., 98 (1985), pp. 247-261.

[136] I. KRA, Automorphic Forms and Kleinian Groups, Benjamin Reading, Massachusetts, 1972.

[137] M. KRECK AND W. LüCK, The Novikov conjecture, vol. 33 of Oberwolfach Seminars, Birkhäuser Verlag, Basel, 2005. Geometry and algebra.

[138] S.L. Krushkal', B.N. Apanasov, and N.A. GusevskiĬ, Kleinian groups and uniformization in examples and problems, vol. 62 of Translations of Mathematical Monographs, American Mathematical Society, 1986.

[139] N. KuIPER, Hyperbolic 4-manifolds and tesselations, Math. Publ. of IHES, 68 (1988), pp. 47-76.

[140] R. Kulkarni, Groups with domains of discontinuity. Some topological aspects of Kleinian groups, Amer. J. of Math., 100 (1978), pp. 897-911.

[141] _ Infinite regular coverings, Duke Math. Journal, 45 (1978), pp. 781-796.

[142] M. LACKenBy, Heegaard splittings, the virtually Haken conjecture and property $(\tau)$, Invent. Math., 164 (2006), pp. 317-359.

[143] Y. LAI. In preparation.

[144] J.-S. Li AND J.J. Millson, On the first Betti number of a hyperbolic manifold with an arithmetic fundamental group, Duke Math. J., 71 (1993), pp. 365-401.

[145] R. Livne, On certain covers of the universal elliptic curve. Ph. D. Thesis, Harvard University, 1981.

[146] A. Lubotzky, Eigenvalues of the Laplacian, the first Betti number and the congruence subgroup problem, Ann. of Math., 144 (1996), pp. 441-452.

[147] J. LUUKKAINEN, Lipschitz and quasiconformal approximation of homeomorphism pairs, Topology Appl., 109 (2001), pp. 1-40.

[148] C. Maclachlan And A. Reid, The arithmetic of hyperbolic 3-manifolds, vol. 219 of Graduate Texts in Mathematics, Springer-Verlag, New York, 2003.

[149] V.S. MAKAROV, On a certain class of discrete groups of Lobačevskiřspace having an infinite fundamental region of finite measure, Dokl. Akad. Nauk SSSR, 167 (1966), pp. 30-33.

[150] - The Fedorov groups of four-dimensional and five-dimensional Lobačevskir space, in Studies in General Algebra, No. 1 (Russian), Kišinev. Gos. Univ., Kishinev, 1968, pp. 120-129.

[151] A. MALĆEv, On isomorphic matrix representations of infinite groups, Mat. Sb., 8 (1940), pp. 405-422. 
[152] A. Marden, Geometry of finitely generated Kleinian groups, Ann. of Math., 99 (1974), pp. 383-496.

[153] - A proof of the Ahlfors finiteness theorem, in "Spaces of Kleinian groups", vol. 329 of London Math. Soc. Lecture Note Ser., Cambridge Univ. Press, 2006, pp. 247-257.

[154] G. Martin, On discrete Möbius groups in all dimensions: A generalization of Jorgensen's inequality, Acta Math., 163 (1989), pp. 253-289.

[155] B. Maskit, A characterization of Schottky groups, J. d' Anal. Math., 19 (1967), pp. 227-230

[156] — On boundaries of Teichmüller spaces and on Kleinian groups, II, Annals of Math., 91 (1970), pp. 607-663.

[157] —, Kleinian groups, vol. 287 of Grundlehren der Math. Wissenschaften, Springer, 1987.

[158] S. Matsumoto, Topological aspects of conformally flat manifolds, Proc. Jap. Acad. of Sci., 65 (1989), pp. 231-234.

[159] - Foundations of flat conformal structure, in "Aspects of low-dimensional manifolds", Kinokuniya, Tokyo, 1992, pp. 167-261.

[160] K. Matsuzaki, Ergodic properties of discrete groups; inheritance to normal subgroups and invariance under quasiconformal deformations, J. Math. Kyoto Univ., 33 (1993), pp. 205-226.

[161] J. Maubon, Variations d'entropies et déformations de structures conformes plates sur les variétés hyperboliques compactes, Ergodic Theory Dynam. Systems, 20 (2000), pp. 1735-1748.

[162] J.P. MCCAMmond And D.T. Wise, Coherence, local quasiconvexity, and the perimeter of 2-complexes, Geom. Funct. Anal., 15 (2005), pp. 859-927.

[163] C. McMullen, Local connectivity, Kleinian groups and geodesics on the blowup of the torus, Invent. Math., 146 (2001), pp. 35-91.

[164] J.J. Millson, On the first Betti number of a constant negatively curved manifold, Ann. of Math., 104 (1976), pp. 235-247.

[165] Y. Minsky, On rigidity, limit sets and invariants of hyperbolic 3-manifolds, Journal of the AMS, 7 (1994), pp. 539-588.

[166] - The classification of Kleinian surface groups, I: Models and bounds. Preprint, 2003.

[167] Combinatorial and geometrical aspects of hyperbolic 3-manifolds, in "Kleinian groups and hyperbolic 3-manifolds" (Warwick, 2001), vol. 299 of London Math. Soc. Lecture Note Ser., Cambridge Univ. Press, Cambridge, 2003, pp. 3-40.

[168] M. MJ, Cannon-Thurston maps, I: Bounded geometry and a theorem of McMullen. Preprint, 2005.

[169] — Cannon-Thurston maps for surface groups, II: Split geometry and the Minsky model. Preprint, 2006.

[170] J. Morgan, On Thurston's uniformization theorem for three-dimensional manifolds, in "The Smith conjecture", J. Morgan and H. Bass, eds., Academic Press, 1984, pp. 37-125. 
[171] Group actions on trees and the compactification of the space of classes of $S O(n, 1)$ representations, Topology, 25 (1986), pp. 1-33.

[172] J. Morgan and P. Shalen, Degenerations of hyperbolic structures, III: Actions of 3-manifold groups on trees and Thurston's compactness theorem, Ann. of Math., 127 (1988), pp. 457-519.

[173] J. Morgan and G. Tian, Ricci flow and the Poincaré conjecture. Preprint, 2006.

[174] L. Mosher, Hyperbolic-by-hyperbolic hyperbolic groups, Proceedings of AMS, 125 (1997), pp. 3447-3455.

[175] G.D. Mostow, Strong rigidity of locally symmetric spaces, vol. 78 of Annals of mathematical studies, Princeton University Press, Princeton, 1973.

[176] S. Nayatani, Patterson-Sullivan measure and conformally flat metrics, Math. Z., 225 (1997), pp. 115-131.

[177] P.J. Nicholls, The ergodic theory of discrete groups, Cambridge University Press, 1989.

[178] K.-I. Ohshika And L. Potyagailo, Self-embeddings of Kleinian groups, Annales de l'École Normale Supérieure, 31 (1998), pp. 329-343.

[179] H. Ohtake, On Ahlfors' weak finiteness theorem, J. Math. Kyoto Univ., 24 (1984), pp. $725-740$

[180] J.-P. OtAL, Le théorème d'hyperbolisation pour les variétès fibrées de dimension 3, Astérisque 235, Société mathématique de France, 1996.

[181] — Thurston's hyperbolization of Haken manifolds, in "Surveys in differential geometry", Vol. III (Cambridge, MA, 1996), Int. Press, Boston, MA, 1998, pp. 77194.

[182] F. PAUlin, Topologie de Gromov equivariant, structures hyperboliques et arbres reels, Inventiones Mathematicae, 94 (1988), pp. 53-80.

[183] G. Perelman, Entropy formula for the Ricci flow and its geometric applications. Preprint, math.DG/0211159, 2002.

[184] - Ricci flow with surgery on 3-manifolds. Preprint, math.DG/0303109, 2003.

[185] R.S. Phillips AND P. SARNAK, The Laplacian for domains in hyperbolic space and limit sets of Kleinian groups, Acta Math., 155 (1985), pp. 173-241.

[186] V. PoÉnARu, Le théorème de s-cobordisme, in Séminaire Bourbaki, 23ème année (1970/1971), Exp. No. 392, Springer, Berlin, 1971, pp. 197-219. Lecture Notes in Math., Vol. 244.

[187] L. Potyagailo, The problem of finiteness for Kleinian groups in 3-space, in "Knots 90" (Osaka, 1990), de Gruyter, Berlin, 1992, pp. 619-623.

[188] - Finitely generated Kleinian groups in 3-space and 3-manifolds of infinite homotopy type, Transactions of the AMS, 344 (1994), pp. 57-77.

[189] M.S. Raghunathan and T.N. Venkataramana, The first Betti number of arithmetic groups and the congruence subgroup problem, in "Linear algebraic groups and their representations" (Los Angeles, 1992), vol. 153 of Contemp. Math., Amer. Math. Soc., 1993, pp. 95-107.

[190] J. Ratcliffe, Foundations of hyperbolic manifolds, Springer, 1994.

[191] J. Ratcliffe And S. Tschantz, Some examples of aspherical 4-manifolds that are homology 4-spheres, Topology, 44 (2005), pp. 341-350. 
[192] F. Raymond, Classification of the actions of the circle on 3-manifolds, Trans. Amer. Math. Soc., 131 (1968), pp. 51-78.

[193] M. REES, The Ending Laminations Theorem direct from Teichmuller geodesics. Preprint, math.GT/0404007, 2004.

[194] A. ReZnikov, Analytic topology of groups, actions, strings and varietes. Preprint, 1999.

[195] E. RIPs, Subgroups of small cancellation groups, Bull. London Math. Soc., 14 (1982), pp. $45-47$.

[196] C. Rourke And B. SAnderson, Introduction to piecewise-linear topology, Springer study edition, Springer, 1982.

[197] K. Scannell, Local rigidity of hyperbolic 3-manifolds after Dehn surgery, Duke Math. J., 114 (2002), pp. 1-14.

[198] R. Schoen And S.-T. YAu, Conformally flat manifolds, Kleinian groups and scalar curvature, Invent. Math., 92 (1988), pp. 47-71.

[199] R. Schwartz, Complex hyperbolic triangle groups, in Proceedings of the International Congress of Mathematicians, Vol. II (Beijing, 2002), Beijing, 2002, Higher Ed. Press, pp. 339-349.

[200] J. Schwermer, Special cycles and automorphic forms on arithmetically defined hyperbolic 3-manifolds, Asian J. Math., 8 (2004), pp. 837-859.

[201] P. Sсотт, Compact submanifolds of 3-manifolds, Journ. of the LMS, 6 (1973), pp. 437-448.

[202] _ Compact submanifolds of 3-manifolds, Journ. of the LMS, 7 (1973), pp. 246250

[203] A. Selberg, On discontinuous groups in higher-dimensional symmetric spaces, in "Contributions to function theory", Bombay, Tata Institute, 1960, pp. 147-164.

[204] Y. Shalom, Rigidity, unitary representations of semisimple groups, and fundamental groups of manifolds with rank one transformation group, Ann. of Math. (2), 152 (2000), pp. 113-182.

[205] J. Stallings, On torsion-free groups with infinitely many ends, Ann. of Math., 88 (1968), pp. 312-334.

[206] B. Stratmann, A note on geometric upper bounds for the exponent of convergence of convex cocompact Kleinian groups, Indag. Math. (N.S.), 13 (2002), pp. 269-280.

[207] D. Sullivan, Hyperbolic geometry and homeomorphisms, in "Proceedings of Georgia Conference on Geometric Topology", Academic Press, 1977, pp. 543-555.

[208] - On ergodic theorey at infinity of arbitrary discrete groups of hyperbolic motions, Ann. Math. Stud., 97 (1981), pp. 465-496.

[209] —- On finiteness theorem for cusps, Acta Math., 147 (1981), pp. 289-299.

[210] - Quasiconformal homeomorphisms and dynamics. Structural stablity implies hyperbolicity, Acta Math., 155 (1985), pp. 243-260.

[211] - Related aspects of positivity in Riemannian geometry, Journal of Differential Geometry, 25 (1987), pp. 327-351.

[212] S. TAN, Deformations of flat conformal structures on a hyperbolic 3-manifold, J. Differential Geom., 37 (1993), pp. 161-176. 
[213] W. Thurston, Geometry and topology of 3-manifolds. Princeton University Lecture Notes, 1978-1981.

[214] — Hyperbolic structures on 3-manifolds, I, Ann. of Math., 124 (1986), pp. 203246.

[215] — Three-Dimensional Geometry and Topology, I, vol. 35 of Princeton Mathematical Series, Princeton University Press, 1997.

[216] P. TukiA, The Hausdorff dimension of the limit set of a geometrically finite Kleinian group, Acta Math., 152 (1984), pp. 127-140.

[217] — On isomorphisms of geometrically finite Moebius groups, Mathematical Publications of IHES, 61 (1985), pp. 171-214.

[218] - Homeomorphic conjugates of Fuchsian groups, J. Reine Angew. Math., 391 (1988), pp. 1-54.

[219] — A survey of Möbius groups, in Proceedings of the International Congress of Mathematicians, Vol. 1, 2 (Zürich, 1994), Basel, 1995, Birkhäuser, pp. 907-916.

[220] P. TUkiA AND J. VÄIsÄLÄ, Lipschitz and quasiconformal approximation and extension, Ann. Acad. Sci. Fenn. Ser. A I Math., 6 (1981), pp. 303-342.

[221] È.B. VinBerg, Discrete groups generated by reflections in Lobačevskǐ spaces, Mat. Sb. (N.S.), 72 (114) (1967), pp. 471-488; correction, ibid. 73 (115) (1967), 303.

[222] Ė.B. Vinberg And O.V. Shvartsman, Discrete groups of motions of spaces of constant curvature, in Geometry, II, vol. 29 of Encyclopaedia Math. Sci., Springer, 1993, pp. 139-248.

[223] S. WANG AND Q. Zhou, On the proper conjugation of Kleinian groups, Geom. Dedicata, 56 (1995), pp. 145-154.

[224] A. WeIL, Discrete subgroups of Lie groups, I, Ann. of Math., 72 (1960), pp. 69-384.

[225] - Discrete subgroups of Lie groups, II, Ann. of Math., 75 (1962), pp. 578-602.

[226] - Remarks on the cohomology of groups, Ann. of Math., 80 (1964), pp. 149-157.

[227] N. Wielenberg, Discrete Moebius groups: fundamental polyhedra and convergence, Amer. Journ. Math., 99 (1977), pp. 861-878.

[228] C. YuE, Dimension and rigidity of quasifuchsian representations, Ann. of Math. (2), 143 (1996), pp. 331-355.

Michael Kapovich

Department of Mathematics,

University of California,

Davis, CA 95616

USA

e-mail: kapovich@math.ucdavis.edu 